\title{
the ofrove
}

working papers on english studies

\section{$21-2014$}
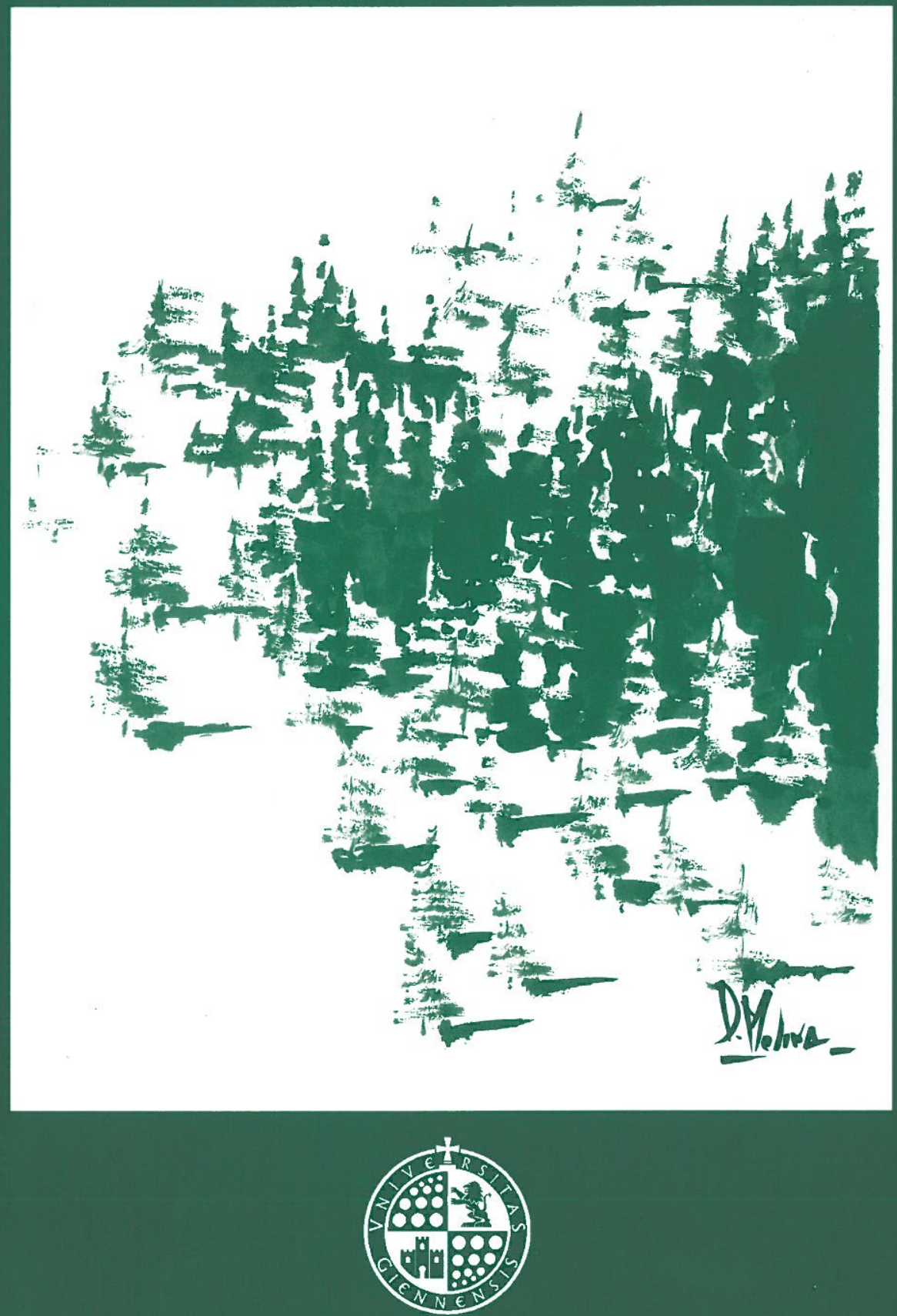

UNIVERSIDAD DE JAÉN 




\section{The Grove}

\section{Working Papers on English Studies}

\section{1}

2014

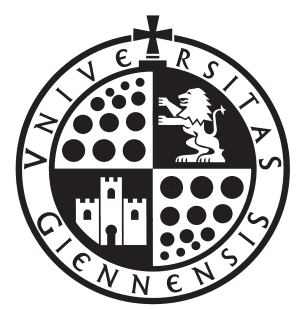

Research Group HUM. 271 (Andalusian Government) Grupo de Investigación HUM. 271 de la Junta de Andalucía 


\title{
THE GROVE. \\ WORKING PAPERS ON ENGLISH STUDIES \\ I.S.S.N.: 1137-005X \\ EDITORS / DIRECCIÓN
}

\author{
General Editor \\ Carmelo Medina Casado (University of Jaén) \\ Managing Editors \\ Jesús López-Peláez Casellas (University of Jaén) \\ Luciano García García (University of Jaén) \\ Book Review Editor \\ José Ruiz Mas (University of Granada) \\ Assistant Editor \\ Cinta Zunino Garrido (University of Jaén) \\ Style Supervisor \\ Ali S. Zaidi (State University of New York - Canton) \\ Technical Secretaries \\ Almudena Machado Jiménez \\ Alejandro Parras Rodríguez
}

\section{Indexed by MLA, IEDCYT-CSIC, Latindex and DICE.}

Published yearly (1 vol. per year) by the Research Group Hum. 271 of the Andalusian Government, Department of English Studies, University of Jaén, Spain / Publicación anual (1 vol. al año) del Grupo de Investigación Hum. 271 de la Junta de Andalucía, en el Departamento de Filología Inglesa de la Universidad de Jaén.

Please address subscriptions and submissions to / Las subscripciones y las propuestas deberán dirigirse a:

Editors / Dirección

The Grove. Working Papers on English Studies

Departamento de Filología Inglesa, Facultad de Humanidades y CC.EE., Edificio D-2, Universidad de Jaén, Campus Las Lagunillas s/n, 23071 Jaén, Spain / España. grove@ujaen.es; Phone / Tlf. +34 953212609 / +34 953212136 / +34 953211818

Copyright@: Grupo de Investigación Hum. 271 de la Junta de Andalucía www.ujaen.es/dep/filing/web_departamento/grove/grove.htm

Front cover design / Diseño de cubierta: David Medina Sánchez - Servicio de Publicaciones de la Universidad de Jaén / Jaén University Press

Legal Deposit / Depósito Legal: J 544-2014

I.S.S.N.: $1137-005 \mathrm{X}$

\section{Distribution / Difusión:}

Publicaciones de la Universidad de Jaén

Vicerrectorado de Extensión Universitaria, Deportes y Proyección Institucional

Campus Las Lagunillas, Edificio Biblioteca

Vicerrectorado de Extensión Universitaria

Campus Las Lagunillas, s/n, Edificio D-2,

23071 JAÉN - Spain

Phone/Tlf. +34 953212355 / +34 953212609

Fax. 953211824 / +34 953212136

\section{Printed by / Impreso por:}

Gráficas "La Paz" de Torredonjimeno, S. L.

Avda. de Jaén, s/n 23650 TORREDONJIMENO (Jaén)

Phone/Telephone: +34 953571087

Fax. 953521207 


\title{
EDITORIAL BOARD / CONSEJO EDITORIAL
}

\section{ADVISORY BOARD}

\author{
John Drakakis (University of Stirling) \\ Fred C. Robinson (Yale University) \\ Juan Fernández Jiménez (Penn State University) \\ James Simpson (University of Cambridge) \\ Jüri Talvet (University of Tartu) \\ Gerardo Piña Rosales (City University of New York) \\ Rüdiger Ahrens (University of Würzburg) \\ Joan Torres-Pou (Florida International University) \\ Susana Cavallo (Loyola University Chicago)
}

\section{SCIENTIFIC BOARD}

Nieves Alberola Crespo (Universitat Jaume I, Castellón)

Alejandro Alcaraz Sintes (Universidad de Jaén)

Patricia Álvares Sánchez (Universidad de Cádiz)

Bárbara Arizti Marín (Universidad de Zaragoza)

Sonia Baelo Alué (Universidad de Zaragoza)

Antonio Andrés Ballesteros González (UNED, Universidad Nacional de

Educación a Distancia)

Antonio Bueno González (Universidad de Jaén)

Yolanda Caballero Aceituno (Universidad de Jaén)

Margarita Carretero González (Universidad de Granada)

Antonio Vicente Casas Pedrosa (Universidad de Jaén)

Susana Cavallo (Loyola University Chicago)

Mónica Calvo Pacual (Universidad de Zaragoza)

Francisco Collado Rodríguez (Universidad de Zaragoza)

Pilar Cuder Domínguez (Universidad de Huelva)

María Luisa Dañobeitia Fernández (Universidad de Granada)

Eva Rosa Darías Beautell (Universidad de La Laguna)

Eroulla Demetriou Demetriou (Universidad de Jaén)

Francisco Javier Díaz Pérez (Universidad de Jaén)

Belén Díez Bedmar (Universidad de Jaén)

Carme Font Paz (Universitat Autònoma de Barcelona)

Paola García Ramírez (Universidad de Jaén)

Felicity Hand (Universitat Autònoma de Barcelona) 
Kurt Harris (Southern Utah University)

Manuel Jiménez Raya (Universidad de Granada)

Isabel Durán Giménez-Rico (Universidad de Complutense de Madrid)

Milagros López-Peláez Casellas (Coventry University)

Gloria Luque Agulló (Universidad de Jaén)

Carme Manuel Cuenca (Universitat de València)

María del Carmen Méndez García (Universidad de Jaén)

José María Mesa Villar (UCAM, Universidad Católica

San Antonio de Murcia)

Francisca Molina Navarrete (Universidad de Jaén)

Andrew Monnickendam (Universitat Autònoma de Barcelona)

Jesús Manuel Nieto García (Universidad de Jaén)

Juan Ignacio Oliva Cruz (Universidad de La Laguna)

Eugenio Manuel Olivares Merino (Universidad de Jaén)

Julio Ángel Olivares Merino (Universidad de Jaén)

Ana María Ortega Cebreros (Universidad de Jaén)

Nieves Pascual Solere (Universidad de Jaén)

María Luisa Pérez Cañado (Universidad de Jaén)

Juan Antonio Prieto Pablos (Universidad de Sevilla)

Juan Ráez Padilla (Universidad de Jaén)

Genara Pulido Tirado (Universidad de Jaén)

Alfonso Jesús Rizo Rodríguez (Universidad de Jaén)

María Elena Ródríquez Martín (Universidad de Granada)

Gerardo Rodríguez Salas (Universidad de Granada)

Christopher Rollason (Independent Scholar, Luxembourg)

José Ruiz Más (Universidad de Granada)

Ana Sáez Hidalgo (Universidad de Valladolid)

María del Pilar Sánchez Calle (Universidad de Jaén)

Francisco Javier Sánchez Escribano (Universidad de Zaragoza)

Adelina Sánchez Espinosa (Universidad de Granada)

Sheri Spaine-Long (University of North Carolina, Charlotte)

Jesús Varela Zapata (Unversidade de Santiago de Compostela)

Rafael Vélez Nuñez (Universidad de Cádiz)

Ali Zaidi (State University of New York at Canton) 


\title{
THE GROVE \\ WORKING PAPERS ON ENGLISH STUDIES
}

21

\author{
CONTENTS
}

Foreword

9

THE CONCEPT OF PERVERSION IN CHUCK PALAHNIUK'S NOVEL CHOKE

Alexandra Bikkyova

“THE SYNAPSES COLLAPSE; WELL, LET THEM..." EL FEMINISMO POSTMODERNO DE THE STONE DIARIES DE CAROL SHIELDS

Macarena Garcia-Avello....

UN CASO NOTABLE DE TRADUCCIÓN INDIRECTA: THE LAST OF THE MOHICANS DE FENIMORE COOPER TRADUCIDO POR VICENTE PAGASARTUNDUA A TRAVÉS DEL FRANCÉS

Ángeles García Calderón, Beatriz Martínez Ojeda..

HOUSES WITH PICKET FENCES: TRYING TO RECOVER A PAST THAT NEVER WAS IN PLEASANTVILLE AND CELEBRATION, FLORIDA

M. Carmen Gómez Galisteo

CAN LITERARY WORKS HELPTO MEMORIALIZE NATURAL DISASTERS? ZORA NEAL HURSTON'S THEIR EYES WERE WATCHING GOD

Aitor Ibarrola-Armendáriz.

"THEIR TRAVELS WERE REAL TRAVELS": HISTORY AND FICTION IN J.M. COETZEE'S "THE NARRATIVE OF JACOBUS COETZE" AND IN EUROPEAN EXPLORATION NARRATIVES IN SOUTHERN AFRICA

María J. López.

VENGEANCE DEFERRED: CHILDREN IN THE SELECTED SHORT STORIES OF SAKI

Sean Moran.

\section{BOOK REVIEWS}

VIORICA PATEA, ED. (2012). SHORT STORY THEORIES: A TWENTYFIRST CENTURY PERSPECTIVE

José R. Ibáñez . 
TIMOTHY M. COSTELLOE, ED. (2012). THE SUBLIME: FROM ANTIQUITY TO THE PRESENT

Jonathan P. A. Sell .................................................................. 147

BIOGRAPHIC NOTES ON THE AUTHORS ........................................... 155

NOTES FOR CONTRIBUTORS........................................................... 159

POEM (TWILIGHT) BY P. PREETHU ............................................. Back Cover 


\section{FOREWORD}

\section{Dear reader:}

Once more the The Grove. Working Papers on English Studies has finally come out at the end of the year. We give heartfelt thanks to our authors and blind referees without whom our endeavour would be fruitless. Referees are the most generous and important part of a scientific journal, as they mainly carry out valuable work gratis et amore. And they are not only beneficial to our journal, but also to the contributors themselves. Indeed, their generally well meant and insightful judgement is an integral part to improving the contributions received, even those that are not finally accepted and which are given useful advice regarding their improvement for further submissions. And what can we say about contributors? The very word underlines the crucial role they play in our journal. Without their contribution our undertaking would not be possible and that is why we want to thank all the authors who have trusted us to assess and diffuse their works.

This year The Grove will appear once again both online and in print. We are paving the way for the complete digitalization of the editing process, and next year, in accordance with the directions of the University of Jaén Publishing Service, it will appear wholly online, which will mean it will be fully digitalized. For the time being digitalization has consisted in offering the Grove in pdf format, including previous issues (see http://revistaselectronicas.ujaen.es/index.php/grove/). We follow the same method for this issue, since this is the easiest and surest way to avoid variance between the online and the printed versions.

As for the articles and reviews constituting the present issue, it seems that the balance has been overwhelmingly tipped to the literary side, and within this literary side, mostly to the ample domain of Modern American literature. Alexandra Bikkyova offers an original and impartial analysis of perversion both as a matter of fiction and reception in the work of Chuck Palahniuk's Choke, while Macarena Garcia-Avello fruitfully explores femininity as an apt agent of postmodernism in The Stones Diary by Carol 
Shields. M. Carmen Gómez Galisteo, gives a very interesting cultural survey of the fabrication of an idealized recent past (the 1950s) in the young USA to scaffold the present both in films and urban development (Houses with Picket Fences: Trying to Recover a Past that Never Was in Pleasantville and Celebration, Florida). In a somewhat similar line, in the sense that this article aptly mixes fiction and urban life (as affected by natural disasters), Aitor Ibarrola-Armendáriz reflects on the way the 1928 hurricane is elaborated into the final chapters of Zora Neale Hurston's Their Eyes Were Watching God. Were it not for the works of María J. López and Sean Moran, who devote their works to the Edwardian H.H. Munro and the 'postcolonial' John M. Coetzee respectively, the whole of our journal would be taken up by North American matters. María J. López presents us with the problems posed by the impossibility of disentangling history from fiction in travelogues as poignantly illustrated in Coetzee's novella "The Narrative of Jacobus Coetzee", drawing very pertinently from Hayden White's and Michel de Certeau's theoretical frameworks, while in a very enlightening essay Sean Moran decodes the clues of the treatment of children in H.H. Munro's short stories. If it were not also for the article by Ángeles García Calderón and Beatriz Martínez Ojeda, which very discerningly disentangle the skein of mediated translation of Fenimore Cooper into Spanish by Vicente Pagasartundua (through the French of Jean-Baptiste Defauconprêt), the whole plot of our small wood would be occupied by literary criticism. Finally, the two terse, well balanced, well informed and very informative reviews that complete the present issue of The Grove by José R. Ibáñez (Viorica Patea's book Short Story Theories...) and Jonathan P. A. Sell (The Sublime from Antiquity to the Present) scrutinize with deep acumen and academic excellence the weighty matters of their respective books.

All this is what constitutes The Grove. Working Papers on English Studies, number 21 for the year 2014. We do not lose hope that our work as editors will serve, albeit minimally, to broaden the field of knowledge in English Studies and that it will be useful to you, reader, somewhere, sometimes, somehow. Till then, please receive our greetings, on a not very cold late Fall, in Jaén, in 2014.

Luciano García García Jesús López-Peláez

José Ruiz Mas

Editors 


\title{
THE CONCEPT OF PERVERSION IN CHUCK PALAHNIUK'S NOVEL CHOKE
}

\author{
Alexandra Bikkyova \\ Pavol Jozef Safarik University in Kosice
}

\begin{abstract}
This article analyses the concept of perversion in Chuck Palahniuk's famous novel Choke. Flowing from the theory of Sigmund Freud who defined perversion as any sexual activity deviating from a heteronormative sexual relation with a procreative aim, Choke encompasses types of sexual perversions such as fetishism, voyeurism, sadism, homoeroticism, rape or promiscuity. As the main character, Victor Mancini, is a sex addict who also performs a masturbatory act defined as auto-asphyxia, his sexual encounters could definitely be called perverse. However, the various examples of perversions entwined within the novel seem to be eschewed by Palahniuk's readers who praise the novel greatly, leaving positive responses on online resources dedicated to the novel's reception. By these means, one could state that perverse literature is perceived as 'cool' nowadays, having the power not only to normalize perversion, but even to make it aesthetic.
\end{abstract}

Key words: perversion, Palahniuk, Choke, Freud, reception.

\section{Resumen}

Este artículo analiza el concepto de la perversión en la famosa novela de Chuck Palahniuk Choke. A partir de la teoría de Sigmund Freud que definió la perversión como cualquier tipo de actividad sexual que se desvía de una relación hetero-normativa encaminada a la procreación, Choke comprende tipos de perversión sexual tales como el fetichismo el voyeurismo, el sadismo, las relaciones homoeróticas, la violación y la promiscuidad. En su papel de personaje principal, Victor Mancini se presenta como un sexo-adicto que además realiza un tipo de 
acto masturbatorio denominado auto-axfixia. Sus encuentros sexuales pueden ser claramente calificados de perversos. Sin embargo, los varios ejemplos de perversión que se entrelazan en la novela parecen ser no tenidos en cuenta por los lectores de Palahniuk que elogian la novela, dejando constancia de una respuesta positiva en los medios online dedicados a la recepción de la novela. De tal manera que se podria afirmar que la literatura perversa se percibe como cool en la actualidad, lo que le da la capacidad no sólo de normalizar la perversión, sino de transformarla en materia estética.

Palabras clave: perversión, Palahniuk, Choke, Freud, recepción.

Choke, widely considered one of Chuck Palahniuk's most successful and controversial works, was published in 2001, and is the author's fourth novel. This novel maintains Palahniuk's tradition of writing extravagant stories that cover such aberrant themes as sexual excess, use of drugs, violence and pornography. The aim of this paper is to focus on two important aspects of Choke: the encoding of perversion in the novel itself, and its decoding by an audience, which will be exemplified by a few brief online responses, at a literary blog website, LibraryThing. com, and two of the most popular social networks, Facebook and Twitter. The choice of the latter aspect resides in the author's interest in the literary reception of perversion on the most modern online platforms. The first aspect examined will be based on a close reading of the novel, depicting the numerous instances of perversion encoded in it, while the second aspect, flowing from reception theory, will attempt to point out that the audience either does not decode these individual instances of perversion in Choke, or if it does, it is not condemned; rather, it is, celebrated. By these means this article could contribute to the existing essays on this particular novel by Palahniuk through a novel approach; that is, by depicting perversion as a phenomenon which attracts a young audience to reading transgressive fiction.

Choke is known and remembered for its bizarre plot that revolves around Victor Mancini, a pervert and sex addict obsessed with choking on food in restaurants, suffering from a so-called 'auto-erotic asphyxia', and his mother Ida who used "the foreskin of Jesus Christ to conceive [him]" (Kavadlo 145), persuading Victor to believe he is "the son of Christ, or possibly Christ himself" (ibid.). Besides the plot, 
Palahniuk's narrative techniques also make Choke a rather unusual and transgressive novel. For instance, the majority of the narrative set in the present is told by Victor, an unreliable, first person narrator, who, due to his status as an addict, makes it difficult to tell whether the facts he claims to be true are indeed trustworthy. Addicts often tend to be liars and sometimes mask their true behaviour with an alternative demeanour, which makes reality sound more acceptable. As perverts know that what they are doing is wrong, they often construct an imaginary reality as a means of getting by in everyday life. Due to the first person narrative, as David Cowart argues, "one cannot easily test the picture Victor Mancini gives of himself, his foster mothers, his work, his world. Is he really an addict? A sex addict?" (Cowart 160) In addition, the present is mixed with passages from the past unfolded by a third person narrator, which informs the reader about Victor's childhood experiences with Ida and his foster mothers. Because of these narrative methods, the temporality of the narrative is not straightforward, and is often interrupted by flashbacks. The present and the past often become fused. Moreover, the reader is warned as early as the first page of the novel to stop reading. Indeed, the narrator states: "If you're going to read this, don't bother. After a couple of pages, you won't want to be here. So forget it. Go away. Get out while you're still in one piece. Save yourself" (Palahniuk 1). The effect of demanding that the implied reader stops reading on page one is actually to further pique the reader's curiosity to continue with the story. As Kavadlo explains, this effect is "at once inviting and distancing" (Kavadlo 147). Due to these techniques utilised by Palahniuk, the novel acquires a thought-provoking nature, coming across as attractive to the reader.

In addition, Palahniuk uses a narrative device expressed by the phrase "See also", which is interpreted by Kavadlo as being used in "a Freudian fashion, to conflate lovers and mother, and sex and death", appearing " 41 times throughout the novel" (ibid. 149). This technique is mainly used to "refer to sexual partners ..., to Paige Marshall, and, in Oedipal confusion, to his mother" (ibid.). For instance, while expressing his feelings toward Paige, Victor explains: "What I want is to be needed. What I need is to be indispensable to somebody. Who I need is somebody that will eat up all my free time, my ego, my attention. Somebody addicted to me. A mutual addiction. See also: Paige Marshall" (213). Thus, all these post-modern narrative techniques tend to attract young audiences and distract them from perverse subjects. 
What is perhaps even more intriguing for the audience than the narrative techniques is the slang language used by Palahniuk, which is represented by such instances as "to get laid" (17), "twerp" (2), "sexaholic chicks" (174), or "Miss Lacey was bobbing on my dog" (183). The author's style is also rich in vulgarisms such as "stupid shit" (8), "fucking corn dogs" (2), "son of a bitch" (177), "piss you off" (2), and it also contains a variety of outrageous themes employed in the narrative. One could consider various types of addiction, love, sex, drugs, disease, the Oedipus complex and choking as some of the most important instances. Indeed, it seems that even the title given to the novel was chosen specifically to shock and arouse the curiosity of the prospective reader. Kavadlo describes the name of the novel and its primary theme in this manner: "Choke': a single word, a simple reflex. Yet immediately, Chuck Palahniuk's semi-onomatopoeic title, a noun and a verb, foregrounds the novel's self-consuming themes: eating, but also the failure to eat" (ibid. 141). In addition, as he states, "since this is a novel by Palahniuk, 'choke' puns on sex and drugs", too (ibid.). Regarding the phonetic sound, Kavadlo explains that "[i]t resembles Palahniuk's own first name and, sounds like 'joke' and is synonymous with 'gag', but it also suggests the surge of emotion ('choke up') that results, paradoxically, in silence" (ibid.). In other words, Kavadlo asserts that the novel "suggests an oral fixation, in keeping with narrator Victor Mancini's entwined obsessions with food and sex", while "[evoking] speech, and at the same time, blockage, and speechlessness" (ibid.). By these means, food, sex and language become the central elements in Choke.

Since Choke is mainly a novel embodying much perversion within the text itself, this will be discussed by using the following quoted passage to illustrate the scale of perversion encoded in the novel. To start with, one of the central perverse elements, Victor's obsession with choking or so-called 'auto-erotic asphyxia', will be analysed. The deliberate choking of Victor seems to follow an identical pattern each time. He starts eating his meal in a restaurant and what follows is described in this manner:

I drink some wine and try to swallow, but the steak's too much. It sits in the back of my throat. I don't breathe. In the next instant, my legs snap straight so fast my chair flies over behind me. My hands go gripping around my throat. I'm on my feet and gaping at the painted ceiling, my eyes rolled back. (48) 
What comes after is Victor's anticipation of one of the restaurant's other clients reaching out and saving him. The reason for his intentional self-harm is explained by his desire to construct heroes of the people who save him. At the same time, it is his expectation that these saviours will feel responsible for him, and later pay him money, so that he can cover his mother's hospital bills. In Victor's words, "Why I do this is to put adventure back into people's lives. Why I do this is to create heroes. Put people to the test ... Why I do this is to make money" (49). The reason why these saviours feel bonded to him is explained by a Chinese tradition "where if somebody saves your life, they're responsible for you forever" (ibid.). In fact, what Victor really wants is to feel loved by them. "Somebody saves your life, and they'll love you forever", he claims (ibid.). He makes people love him and does not care about them afterwards. So, not only does he fool the audience by staging his own death, he also takes advantage of them by deliberately making them feel responsible for saving his life. In other words, he uses people for his own welfare.

The act of choking is not only a tool to collect thousands of dollars from strangers, nor is it a mere impossibility to swallow food which could have fatal consequences. More disturbingly, choking is associated with Victor's sexual perversity. As choking, which invokes Victor's sexual arousal, is a result of consuming food, his perversion follows the pattern developed by Freud, that is, the association of libido with "nutrition and hunger" (Stoller 14). To clarify, according to Stephen J. Hucker, autoerotic asphyxia or hypoxyphilia "is a paraphilia which is a subcategory of sexual masochism" and a "potentially lethal sexual practice [which] refers to sexual arousal that is produced while reducing the oxygen supply to the brain" (Hucker). Individuals that perform asphyxia tend to keep it a solitary act and, as Hucker demonstrates, the most common techniques used are "self-hanging, strangulation, choking, suffocation and techniques to restrict breathing movements" (ibid.). In other words, choking, or the deliberate failure to consume food, is a type of method of masturbation performed by Victor in order to feel sexually aroused in front of an audience. Since auto-erotic asphyxia is a sub-category of masochism, what Victor engages in, in Freud's terms, is one of the most abundant types of perversion (Freud 23). Moreover, because it is not a solitary performance for Victor, as Nieves Pascual points out, this masochistic act places the audience in the position of voyeurs (Pascual 
181), while voyeurism stands for another perversion in Freud's theory. In one of the novel's passages, Victor describes this bodily act through these lines:

From not breathing, the veins in my neck swell. My face gets red, gets hot. Sweat springs up on my forehead. Sweat blots through the back of my shirt. With my hands I hold tight around my neck, the universal sign language of someone choking to death. (48)

As Pascual suggests, the above description of Victor may be regarded as a parallel to a sexual act and the subsequent attainment of orgasm, as "Victor's neck/shaft swells as with erection, the face/glans becomes tumescent and sweat/semen is emitted" (Pascual 181). As she asserts, his "oral tract is obstructed through food, which usurps the position of the phallus as the ultimate source of pleasure, causing autoerotic asphyxia" (ibid.). Choking, as one of the absolute springs of pleasure for Victor, is repeated throughout the whole novel, and therefore becomes omnipresent. As it represents a link between food and libido, it symbolizes the ultimate life principle defined by Freud (ibid. 14). Moreover, as choking is a type of masochism, which in Victor's case requires an audience, choking might be regarded as perverse from various points of view.

However, Victor is not only a public masturbator, an exhibitionist and therefore a pervert; he also draws sexual pleasure from frequent intercourse with several women that he meets in his sex addiction support group. Even though his sexual encounters are always heteronormative, and thus "normal" and predominantly believed to be non-perverse by Freud, they nevertheless acquire a level of perversity. The reason for this fact lies either in the frequency of the sexual act, in the number of sexual partners, or in the way and place where intercourse is performed. As Freud considered everything besides a heteronormative sexual intercourse with an aim to procreate as perverse (ibid. 16), in Freud's perception all of Victor's sexual intercourses personify perversion. Put differently, neither the sexual aim, nor the sexual objects of Victor fulfil Freud's standards of normalcy.

In one instance Victor role-plays a rape scene with a woman named Gwen. Even though the sex is only a simulation of a rape, it is presented as real. The scene includes a knife, an electrical tape and pantyhose over Victor's face, because Gwen wants him "to be a faceless 
attacker" (171). As rape takes the place of the normal sexual act, Gwen's obsession with rape-simulating sex clearly embodies a fetish, another perversion defined by Freud (Freud 19). What concerns Victor, however, is not the fact that he is going to role-play raping a woman, rather he is worried about the embarrassment of buying a pair of pantyhose. In his words, "[a] guy buying pantyhose is either a criminal or a pervert" (172). Put another way, buying a woman's accessory makes him feel like a criminal or a pervert but he does not think about the fetishist rape/ sex scene with Gwen either as a crime, or as perversion. Therefore, he is totally normalizing the role-playing of rape. After Victor discovers that Gwen is wearing a female condom, he admits that he "thought rape was supposed to be more spontaneous, you know, a crime of passion" (174), where he again refers to a crime. Subsequently, Gwen seeks to get him into character by proposing that "[a] good rapist will plan his crime meticulously. He ritualizes every little detail. This should be almost like a religious ceremony. What happens here, Gwen says, is sacred" (ibid.). In other words, such a fetish as the sexual role-play of rape is allegorized to a religious ceremony, a sacred act, and therefore, it is not only normalizing but also consecrating perversion. Following Victor's clumsiness in the rape role-play, Gwen becomes angry and dissatisfied: "Why do I do this? Why do I always pick the guy who just wants to be nice and conventional? ... Just one time, I'd like to have an abusive relationship. Just once!" (175). Moreover, before sex, Victor is asked to sign a paper with Gwen's requirements: "Rape is about power. It is not romantic. Do not fall in love with me. Do not kiss me on the mouth. Do not expect to linger after the act. Do not ask to use my bathroom" (italics in original, 173). During the act, what Gwen wants is expressed in this manner: "I want you to hit me.' She says, 'But not too hard and not too soft. Just hit me hard enough so I come"' (ibid.). Hence, in Choke, it is the abusive, perverted, promiscuous relationships filled with sadism, masochism and fetishism that are attractive, not the conventional ones. Romance with all of its characteristics is old-fashioned. What is preferred is violence and perverted sex. Therefore, sex portrayed in Choke is the opposite of normal, in Freudian terms.

When discussing promiscuity and sexual perversion, another woman Victor frequently has sex with, Nico, must be introduced. While having intercourse in the ladies' restroom of a church with her one day, Nico asks Victor about "all the other women" (italics in original, 16) that 
he has had sex with. He replies: "For a complete list of sexual partners, I'd have to check my fourth step. My moral inventory notebook. The complete and relentless history of my addiction" (ibid.). The fourth step refers to one of the twelve steps one has to take in order to become healed in the sexaholics group. As he also admits, he attends the group because, for him, it is a "terrific how-to seminar", or put another way, "Tips. Techniques. Strategies for getting laid you never dreamed of. Personal contacts" (ibid.). Moreover, here he can also take advantage of "the jail girls out for their three hours of sex addict talk therapy. Nico included" (ibid.). Namely, "Wednesday nights mean Nico. Friday nights mean Tanya. Sundays mean Leeza" (ibid.). Undoubtedly, this speaks of his promiscuity and proves his addiction to sex.

Interestingly enough, as Victor usually addresses the members of the sex addiction group with the pronouns 'they' or 'you', he seems to exclude himself from them. "All these people you think are a big joke ... These are sexual compulsives" (12), he explains. He denotes them as "peeping Toms. The nymphos. The dirty old men. The restroom lurkers. The hanballers" (14). Since he distances himself from this group and has as a main objective to get sexually involved with as many women as possible, one could really doubt whether he is a sexual addict or whether he is only playing with women. Nevertheless, in any case, his behaviour certainly is perverse. However, he defends his status of a pervert by arguing that "being a pervert here is not your fault. Compulsive sexual behavior is not about always getting your dick sucked. It's a disease. It's a physical addiction ..." (17). Indeed, Freud did regard perverts as sick (Freud 32), thus Victor describes himself quite aptly. He excuses his status as a pervert by comparing sex addiction to drug addiction. Once you are addicted, it is not easy to escape: "For a sex addict, your tits, your dick, your clit or tongue or asshole is a shot of heroin, always there, always ready to use. Nico and I love each other as much as any junkie loves his fix" (18). On the other hand, being a pervert is a comfortable state to be in for Victor and he has no intention of escaping it. In his opinion "people who say they want a life free from sexual compulsion, I mean forget it. I mean, what could ever be better than sex?" (19). For Victor, sex is a saviour, enabling him to forget his real life problems momentarily. It saves him from loneliness and as he admits, "[t]hese are the only few minutes I can be human" (20). Thus, he reaffirms Freud's premise of libido being analogous to life. 
One could state that the source of Victor's sexual perversion might flow from his childhood experience, when he was acquainted with online pornography from an early age. The type of pornography that he came across is a rather non-standard variety. The narrator informs the readers that Victor as a little boy had a favourite pornographic website which was "pretty much not sexy" (36), because it portrayed photographs of a bent over older man dressed in a Tarzan suit having roasted chestnuts inserted into his anus by a monkey. The importance of this image is unquestionable, since it reappears on several occasions in the novel. The perversion of this particular type of pornography lies in the fact that "in a world where everybody had to look so pretty all the time, this guy wasn't. The monkey wasn't. What they were doing wasn't" (37). Even though pornography itself is considered a type of perversion, the particular website with the Tarzan and the monkey stands for perversion within perversion. Pornography is recognized as a type of voyeurism and since the sexual relation between the man and the monkey forms the basis for evoking sexual arousal, it represents zoophilia, falling within the scope of the worst of perversions defined by Freud (Freud 14). Since little Victor was willingly searching for pornographic websites displaying zoophilia on the internet on a daily basis, his act is in unison with the theory of Freud who believed that love and the search for it is present even in the most terrible of perversions. In addition, Victor apparently did not look up the website for the purpose of sexual arousal. On the contrary, the narrator claims that

[i]t's not the sex part of pornography that hooked the stupid little boy. It was the confidence. The courage. The complete lack of shame. The comfort and genuine honesty. The up-frontness of being able to just stand there and tell the world: Yeah, this is how I chose to spend a free afternoon. Posing here with a monkey putting chestnuts up my ass. (italics in original, ibid.)

As the elements of shame and disgust are seen as direct opposites to libido, it really seems that what Victor admired in the Tarzan/ monkey scene was the opposite of erotic content. Little Victor imagined this is how 'Nirvana' and 'Freedom' ought to look like and thought "If I could do this, I could do anything" (italics in original, 38). The man from the pornographic photographs became Victor's hero, helping him to overcome troublesome situations by a mere thought of him, and hence representing his addiction. As Pascual proposes, "addiction 
provides direction to Palahniuk's characters and fills the holes in their identities" (Pascual 175) and Victor's personality was definitely filled by this perverted pornographic image from his early childhood.

This hole in Victor's identity originated from his relationship with his mother, Ida. Due to the fact that she was believed to be mentally unstable and unable to raise a child alone, she was placed in mental institutions, and Victor was raised by several foster mothers. At times when he needed his mother, he would recall the aforementioned pornographic webpage. In Pascual's opinion, the image of the Tarzan and the monkey helped him "neutralize the feelings of loneliness, sadness and fear" which were caused by "the trauma of his mother's absence" (ibid. 178). In fact, the relationship of Victor and Ida forms another type of perversion in the novel, based on Freud's notion of the Oedipus complex. Victor informs the reader that

[e]very son raised by a single mom is pretty much born married. I don't know, but until your mom dies it seems like all the other women in your life can never be more than just your mistress. In the modern Oedipal story, it's the mother who kills the father and then takes the son. And it's not as if you can divorce your mother. Or kill her. (15-16)

It is very true that all the women Victor has some connection with are only his mistresses, and their relationship is based on sex. Ida seems to be the only important woman in his life, for whom he apparently performs his choking, and whom he, in the end, kills. This fact represents another reversal of Freud's Oedipus complex, where the son wants to kill his father. However, the father figure is missing from his life. As he was raised by a single mother, he never met his father. In compliance with Freud's theory of the Oedipus complex, in order for a little boy to retain his masculinity, he must overcome the castration complex and substitute the love for his mother with a love towards another woman in his adulthood. However, since there is no father figure in Victor's story, he has no one to compete with and does not suffer from castration anxiety. Therefore, no woman can take the position of his mother. Victor truly loves only Ida, while the other women serve as instruments to satisfy his obsession with sex. For instance, he refers to the Oedipus complex when a nurse addresses his mother as Mrs. Mancini. He says: "My mom's not married, unless you count me in that creepy Oedipal way" (225). As Kavadlo argues, "Palahniuk rewrites the Oedipal story; 
with the modern father pre-emptively absent, the mother consumes, not consummates, the relationship with the son" (Kavadlo 148). To illustrate this, Ida chokes on the pudding served to her by Victor, and as a consequence, she dies. Therefore, the relationship with her son is not concluded by Victor's separation from her, as in the original expected outcome of Freud's Oedipus complex. On the contrary, she consumes the food offered by her son, and so her death is a consequence of this consummation. Cowart sees a symbol arising out of Victor killing Ida by asserting that "[h]er death is the ironic consummation of the choking charade" (Cowart 168). As Victor is trying to earn money to keep his mother alive by choking on food throughout the novel, it is ironic that in the end Ida passes away as a result of choking on Victor's food. By these means Victor becomes a 'widower', even if "it isn't the right word, but it's the first that comes to mind" (270).

In this strange rewritten version of the Oedipus complex the father is absent, and thus, as Pascual argues, the whole novel is a quest for Victor to find him (Pascual 180). Not only does he strive to find the father figure represented by the restaurant saviours of his choking by whom he wants to be loved, but he also identifies with the Tarzan man, who by means of "offering his anus is Victor's imago of his father" (ibid.). Thus, it could be affirmed, that the Tarzan "bent over saves him" (ibid.). What Pascual suggests is that the whole search for his father is therefore a parody of Freud and the Oedipus complex. This parody reaches even greater dimensions, when the reader learns of a rather absurd story about Victor's conception. Dr Paige Marshall, after her translation of Ida's diary, proposes that Victor is his own father. Specifically, she states: "Well, if you believe in the Holy Trinity, you're your own father" (145). In other words, according to Paige, Ida is "so delusional she truly believes you're [Victor] the second coming of Christ" (146). This absurd proposition is logically explained in detail the following way:

My mom had gambled on some new kind of fertility treatment. She was almost forty. She wasn't married, she didn't want a husband, but somebody had promised her a miracle. This same somebody, they knew somebody who'd stolen a shoe box from under the bed of a priest. In that shoe box was the last earthly remains of a man. Somebody famous. It was his foreskin. (152)

Hence, Victor is made to believe that the famous man's foreskin from which he was conceived was the foreskin of Jesus Christ. The 
mystery of finding his father was uncovered and the Father had been found. Victor is his own father, and hence, he is God. However, Victor himself does not truly believe in this story and his aim is to persuade Paige about this. He claims: "I'll prove to her I'm no Jesus Christ ... What I am is a dirty, filthy, helpless sexaholic, and I can't change, and I can't stop, and that's all I'll ever be. And I'll prove it" (156). Another time, he argues: "I'm the child of a lunatic. Not a child of God" (ibid.). Nevertheless, his doubts are undisputable and he is not sure what the truth is and this keeps his mind distracted again during sex: "that Jesus idea was just killing my hard-on" (164). He was therefore living a double life, like many other characters of perverted fiction, the one of a sex addict and the one of the second coming of Christ. As with manifold characters of perverted fiction, the double life of Victor offers a more moral side to his perverted acts, and allows him to remain in secrecy. Perverts are usually aware of their perversion, and on that account try to maintain this double life in order to trick their audience by their looking 'normal'.

This double character of Victor is also expressed by the narrator's omnipresent question towards the implied reader: "What would Jesus do/What would Jesus NOT do?", while the second question of the pair reappears countless times until the end of the story. In Cowart's opinion, "this little epithet does something more than declare a disinclination to imitate Christ: it invites ironic recognition that there are in fact things that Jesus would not do" (Cowart 169). To exemplify, the phrase is usually used in a very clever way to amuse the reader during extravagant passages. For instance, during Victor's role-play rape scene with Gwen, she asks him not to stain her bed linen with his semen. However, the opposite happens and his "white soldiers start to toss, and maybe by accident they misjudge the trajectory and toss across her pink bedspread ... What would Jesus NOT do? Spunk graffiti. 'Vandalism isn't the right word, but it's the first word that comes to mind"' (177). Another instance is provided by Victor's sexual intercourse with a woman named Leeza. During sex she asks him "Just what are you trying to prove?", to which Victor replies: "That I'm an unfeeling jerk. That I really don't care. What would Jesus NOT do?" (italics in original, 210).

On the other hand, as Kavadlo argues, Victor "is also doing what Jesus would do" (italics in original), because he "allows himself to 
be consumed so that others may be saved, even when some of those others believe that they are saving him" (Kavadlo 150-151). Specifically, various patients at St Anthony's accuse him of numerous crimes that they believe he committed. Instead of arguing, however, Victor takes the blame. In Kavadlo's words, he "deliberately seems to be doing his worst" (ibid. 149), which is a common tendency in transgressive or perverse novels. During one of his visits to Ida, Victor describes the situation in the hospital in this manner: "Two smiling old ladies wander past us, and one points and says the other: 'There's that nice young man I told you about. He's the one who strangled my cat'. The other lady, her sweater is buttoned wrong, and she says, 'You don't say'. She says, 'He beat my sister almost to death one time"' (115). Another time, Victor starts confessing even more crimes that he did not commit to Mrs Tsunimitsu: "I'm sorry. Sorry I fucked her cat. Sorry I drove over her flower beds. Sorry I shot down her husband's fighter plane. Sorry I flushed her hamster down the toilet. I sight at her and said: 'Did I forget anything?" (154). What he is doing is "simply, freely, willing to martyr himself", but at the same time he also "rebels against the possibility that he may be God, or even good" (Kavadlo 150). He is even arrested following a patient's false accusations of Victor raping her. As Victor states, he does it because he "want[s] to be someone's constant savior" (118), and moreover, he even once admits: "I know that I'm Jesus Christ" (268). Thus, there is a constant confusion between him acting like Jesus Christ by his "sadistic, even pathological" (Kavadlo 150) admissions of untrue guilt and passages of him acting in a 'what would Jesus not do' way. According to Alex E. Blazer, Victor is a representative of "postmodern martyrdom" because he is "both a nihilist ... and a narcissist who unconsciously believes he can save the empty world..." (italics in original, Blazer 143). Therefore, in Blazer's view, "[while] Victor should be pitied, he must neither be trusted nor promoted for he is the product of a delusional mother, hyper-real job, and inauthentic culture who has consistently and compulsively chosen to construct simulated shrines to his own perceived martyrdom" (ibid. 155).

When arrested for a fake admission of raping a ninety-yearold woman, Eva Muehler, Victor sits in the investigation room with detectives, with his anus blocked by two rubber balls which were inserted there during another moment of perverted sexual play the previous day by a girl named Tanya. Now that he had killed his 'mother' and that 
Paige is revealed to be a patient, not a doctor, his life is completely losing its meaning. Therefore, Victor decides to really choke himself to death. However, after a Heimlich manoeuvre performed by one of the detectives, his clogged anus is set free, the rubber balls get released and Victor's excrement covers the room. He describes the happenings as his "entire private life made public. Nothing left to hide. The monkey and the chestnuts" (287) and the reason for this, in Kavadlo's opinion, is that "Victor needs all his shit, literally and figuratively, to come out" (Kavadlo 153). As Cowart concludes, this ending, or "the clearing of the blockage, according to his [Victor] scatological metaphor, takes on additional meaning of considerable importance to contemporary discourse and modern aesthetics" (Cowart 174). What Cowart suggests is that "the reader is invited to think about a discourse that is not "full of it' or 'bogus'- a discourse, in short, that is not ironic" and to recognize Palahniuk's "sardonic recycling of Freudian case history and millennial Christology" which "reveals more ideational substance, in the end, than his critical disparagers might lead one to expect" (ibid.).

As to the decoding of the novel, generally the reader tends to regard the aforementioned instances of perverse behaviour extremely positively. To illustrate this, on the social website LibraryThing. com, which is described as a "community of 1,700,000 book lovers" with entries from authors, publishers, libraries and cover designers (Spalding), several entries prove the immense popularity of the analysed novel. Each review also encompasses a rating of the reviewer, with an average rating for the novel being 3.6 stars out of five and the final ranking result is calculated out of 2872 ratings. In order to portray the main tendencies in decoding Choke, specific audience responses will now be related and a few brief readings of Choke on the aforementioned website will be pinpointed.

A reader using a nickname "rkeipper84" reviewed the novel in a way that might be considered as the prevalent tendency in most of the given entries on the website. Namely, the reviewer admitted to being actually nauseated by the novel. This, however, did not discourage him/her from proceeding to read it. On the contrary, it made them appreciate the novel even more. In fact, the reader states: "This may be one of the only books that has ever made me feel physically ill ever. The gross out factor is a little overwhelming to say the least, but in true Palahniuk fashion, it was intended to have that effect" (ibid.). Interestingly, the perversion 
of the book is not condemned, but celebrated and thus aesthetically acclaimed. As long as its author is Palahniuk, it is admissible to feel nauseated by his work, as that is exactly what one expects from this writer. The interpretation continues in this manner: "As much as I find myself revolted by the images he plants in my mind, I continue to devour his work and it leaves me craving more. Any author that can be that out there and that original deserves the praise" (ibid.). The same stance is articulated by "EmScape" who claimed that "[d]espite being instantly disgusted by a lot of this book, I still found it entertaining. I will read anything this man writes" (ibid.). Likewise, "happyscrapyhero" posted an entry commenting: "loved it. weird... but i [sic] couldn't put it down" (ibid.), leaving a five star rating. The same rating was shared by "sanddancer" who asserted that "[t]he book is full of flawed people who you shouldn't like, but who I ended up really caring about" (ibid.). Based on these opinions, the effects of reading the novel are both the evoking of nausea, and the piquing of the readers' curiosity. Therefore, one could conclude that perversion in the novel is the factor which at times arouses the interest of the readers and persuades them to praise the novel.

While the abovementioned blog instances recognize perversion in Choke and even consider it intriguing, the responses on the social networks Facebook and Twitter do not show traces of its decoding at all, since the novel's reception is only positive. While searching for Choke on Facebook, one comes across the official Facebook page of the novel, which is liked by almost 200,000 people. Most of the posts by Palahniuk's Facebook fans include their acknowledgement of the author's writing in general. These vary from their own artwork symbolizing the novels, to their tattoos with either Palahniuk's quotes or pictures based on them, or "selfies" of people holding copies of Palahniuk's novels in their hands. There are hundreds of fan entries on Palahniuk's wall to wish him happy birthday or Merry Christmas; the fans thank him for inspiring them, or simply share their views on his novels. For example, Jason Knowles shared the following comment on Palahniuk's wall in 2013 saying "Thank you Chuck... You've done more for my life than you know...." (Facebook/Chuck Palahniuk). Another post by Camryn Battiparano exclaims: "I would sell my soul for your autograph" (ibid.). Perhaps due to such interactivity between Palahniuk, his books, and the audience, "The Cult" of Palahniuk's fans has been established, which is also the 
name under which his official website, chuckpalahniuk.net, operates. A similar phenomenon might be noticed on Twitter, where over 500,000 followers of Palahniuk "tweet" their favourite quotes or book reviews with a hashtag "\#Choke" or "\#Palahniuk". One of the examples was posted by "Kyle" who mentioned Choke in her post: "If you've never read 'Choke', 'Invisible Monsters' or 'Survivor' by Chuck Palahniuk then you need to. Right. Now. \#sogood" (Twitter). In addition, "Michaela Jane MJ" tweeted the following about the novel: "Because nothing is as perfect as you can imagine it 'Chuck Palahniuk' - Choke (finished! interesting! messed up! questioning! a good book!)" (ibid.). The remaining tweets share the same positivity.

In conclusion, despite the fact that the respondents on the analysed blog website try to defend themselves by stating "I learned things I wish I didn't know" or "I ended up really caring about" the deviant characters who "you shouldn't like" (Spalding), in the end they do want to know those things and they do love these characters. Most of the time, the reviews are rather terse and do not specify any kind of particular perversion inherent in the novel. Neither the masturbation method of Victor's choking, nor the rewritten Oedipus complex are acknowledged. It seems that the reviewers understand that the novel is perverted, but nevertheless they do not express their thoughts on the particular kinds of perversion that they found disturbing. In other words, perversion is generally understood as the factor which contributes to the novel's aesthetic value, and by these means it is glorified. Perversion therefore is decoded as "cool" and as something that people know that is present in reality, therefore "very, very real" (ibid.). Yet, it is not openly discussed in real life, where the public might unveil their identity behind the aforementioned nicknames. On the other hand, on the internet, where one can freely express their opinions independently and disguise their true identity, people confess their sympathy towards perversion. What Palahniuk does is articulate circumstances that are normally not spoken of. Based on the high percentage of positive rankings of the novel, the audience proves to display their desire to read about perversion and highlight what Tatyana Shumsky calls a "warm rapport Palahniuk's texts establish with their readers" (Shumsky 56). This warm rapport is demonstrable on Palahniuk's online fan sites such as Facebook and Twitter, where "the cult" devoted to Palahniuk is growing day by day. 


\section{WORKS CITED}

Cowart, David. “Anger, Anguish, and Art: Choke.” Collado-Rodríguez, Francisco. Chuck Palahniuk: Fight Club, Invisible Monsters, Choke. London: Bloomsbury, 2013.

Facebook/Chuck Palahniuk. 2014. 23 April 2014. <https://www.facebook.com/ chuckpalahniuk>.

Freud, Sigmund. Three Essays on the Theory of Sexuality. USA: BasicBooks, 1975.

Hucker, Stephen J. Forensic Psychiatry. 2011. 16 April 2014. <http://www. forensicpsychiatry.ca/>.

Kavadlo, Jesse. "Chuck Palahniuk's Edible Complex." Collado-Rodríguez, Francisco. Chuck Palahniuk: Fight Club, Invisible Monsters, Choke. London: Bloomsbury, 2013.

Palahniuk, Chuck. Choke. London: Vitage Books, 2003.

Pascual, Nieves. "Addiction in Choke." Collado Rodríguez, Francisco. Chuck Palahniuk: Fight Club, Invisible Monsters, Choke. London: Bloomsbury, 2013.

Shumsky, Tatyana. "Tracking Conversion: A Structural Analysis of Survivor and Choke." Sartain, Jeffrey A. Sacred and Immoral: On the Writings of Chuck Palahniuk. Newcastle upon Tyne: Cambridge Scholars Publishing, 2009. 40-60.

Spalding, Tim. LibraryThing. 2014. 22 April 2014. http://www.librarything.com.

Stoller, Robert J. Perversion: The Erotic Form of Hatred. New York: Pantheon Books, 1975.

Twitter. 28 June 2011. 23 April 2014. <https://twitter.com>.

Received: 16 June 2014

Accepted: 31 October 2014 



\title{
"THE SYNAPSES COLLAPSE; WELL, LET THEM..." EL FEMINISMO POSTMODERNO EN THE STONE DIARIES DE CAROL SHIELDS
}

\author{
Macarena Garcia-Avello \\ University of Maryland
}

\begin{abstract}
This article examines the way in which postmodern metafiction in Carol Shields's The Stone Diaries can be understood as a starting point to explore the relational and fragmentary subjectivity from a feminist standpoint. Although most analysis on The Stone Diaries put an emphasis on the importance of feminism, the influence of postmodernism in Shields's novel has been commonly overlooked. This article aims to delve into the interaction between feminism and postmodernism in order to demonstrate how this interplay provides the novel with a political function.
\end{abstract}

Key words: postmodernism, feminism, politics, The Stone Diaries

\section{Resumen}

Este artículo examina la manera en que la metaficción postmoderna de Carol Shields en The Stone Diaries sirve de punto de partida para una crítica feminista desde la que se explora el carácter relacional y fragmentario del sujeto femenino. Aunque la crítica haya incidido en la vertiente feminista de la novela de Carol Shields The Stone Diaries, el papel que juega el postmodernismo en la crítica feminista ha pasado desapercibido. El objetivo de este artículo es, por tanto, ahondar en la manera en que el feminismo se intercala con el postmodernismo en la obra de Shields. A lo largo de este trabajoo se demostrará cómo la confluencia entre la condición postmoderna y la construcción de la subjetividad de la protagonista proporciona a la obra una función política. 
Palabras clave: postmodernismo, feminismo, política, The Stone Diaries

Influida por el postmodernismo y el feminismo, The Stone Diaries es un híbrido entre la autobiografía, la biografía, las memorias y la ficción. En ella la protagonista, Daisy Goodwill Flett, "a middle-class woman, a woman of moderate intelligence and medium-sized ego and average good luck" (The Stone 252) se presenta como sujeto y objeto de una representación interceptada por las voces y vidas de los otros, las lagunas y silencios, las posibilidades irrealizadas y el influjo de lo imaginario, en lugar de lo verídico. La novela se divide en una serie de capítulos que hacen referencia a las distintas fases que atraviesa a lo largo de su vida: "Birth, 1905", "Childhood, 1916", "Marriage, 1927", "Love, 1936", "Motherhood, 1947”, "Work, 1955-1964", "Sorrow, 1965”, "Ease, 1977", "Illness and decline, 1985” y, finalmente, "Death". A pesar de que el título alude a las memorias de Daisy, las intromisiones en cada una de estas etapas de las historias y perspectivas de familiares, amigos y conocidos la desplazan constantemente del centro narrativo. La configuración y organización de la narración dejan en evidencia cómo su biografía está marcada tanto por los factores socio-económico y culturales como por las relaciones que establece con quienes la rodean.

Este artículo examina la manera en que la metaficción postmoderna de Shields sirve de punto de partida para una crítica feminista desde la que se explora el carácter relacional y fragmentario del sujeto femenino. Aunque la crítica haya incidido en la vertiente feminista de la novela, el papel que juega el postmodernismo en la crítica feminista ha pasado desapercibido. Por ejemplo, Wendy Roy reconoce que la autora "turns autobiography into critical practice by engaging with feminist theories of life writing [...] At the same time, it questions the efficacy of the discontinuos and relational nature of autobiography as proposed by feminist theories, and provides a critique of social structures that define both women's writing and their lives" (114). El objetivo de este artículo es, por tanto, ahondar en la manera en que el feminismo se intercala con el postmodernismo en la obra de Shields. A lo largo de este se demostrará cómo la confluencia entre la condición postmoderna y la construcción de la subjetividad de la protagonista proporciona a la obra una función política. 
The Stone Diaries cumple una función política relativa al papel de la narración auto/biográfica para representar la experiencia femenina, pues en palabras de la propia Shields, contiene "the lives of women whose stories have more to do with the texture of ordinary life and the spirit of community than with [the] personal battles, goals, and prizes [...] of a male literary tradition" (The Golden Book 249). La función política que subyace a la representación de la vida de una mujer de clase media en Estados Unidos desde los años veinte hasta finales de los setenta se relaciona con la política de representación del yo.

Este artículo comienza con un análisis de la política de representación de Daisy, cuyo objetivo es reinscribir la subjetividad y experiencia femenina, no sin llevar a cabo una revisión muy crítica de las condiciones materiales que han contribuido a ellas. Por tanto, la revalorización de la feminidad alterna con la denuncia de la discriminación padecida por el sector al que pertenece Daisy Goodwill. La política de representación abarca las implicaciones éticas y políticas de la auto/biografía, concebida como "a historically situated discourse of self-representation" (Smith, Watson 14). Teniendo en cuenta esta definición, la narración de la protagonista se presta a un estudio de una subjetividad situada simultáneamente dentro y fuera de la ideología de género. Es decir, un sujeto femenino configurado en relación con los códigos culturales de género, pero capaz, a su vez, de distanciarse críticamente y desmontar esta construcción. Este reconocimiento del género como construcción tiene el efecto de 'politizar' al sujeto, como sucede en la novela de Shields.

Con la representación de Daisy se pretende llenar un vacío, al tiempo que se censura la invisibilización de las vidas de mujeres. El repaso a la política de representación de Daisy supone el primer paso hacia la política de Carol Shields, quien atribuye a su novela una doble función consistente en denunciar y redimir una ausencia:

In many ways, [Daisy] is like so many women of this century who became, in fact, nothing. Their lives did not hold many choices. They were this huge army of women, they were mainly voiceless, they were defined by the people around them. And that became the trick of writing [The Stone Diaries], to write a biography of this woman's life -but it's a life from which she herself is absent. (Randon Illuminations 51) 
La marginalidad de Daisy no aparece idealizada, sino que sirve para cuestionar y deconstruir tanto la identidad hegemónica masculina como las condiciones materiales que han contribuido a la discriminación de las mujeres. La identidad femenina se configura de acuerdo a una serie de discursos que la novela expone y denuncia por haber contribuido a la subyugación de las mujeres pertenecientes al grupo social de la protagonista. Factores como la clase, la raza o la sexualidad de la protagonista imponen un modelo de feminidad atravesado por los discursos de la domesticidad, la división entre el dominio público y el privado que se vincula a la construcción de las subjetividades masculinas y femeninas o la identidad relacional.

La representación de la domesticidad en la novela sirve a dos objetivos aparentemente opuestos, la recuperación narrativa y la crítica. Las numerosas descripciones pormenorizadas de todo lo que concierne al hogar señalan la necesidad de revalorizar esta dimensión de la vida humana que durante siglos se ha visto relegada a los márgenes y a lo insignificante por tratarse del ámbito asociado a las mujeres. Asimismo, la narración arroja una mirada crítica hacia los imperativos de domesticidad. Si bien la esfera doméstica se ve dotada de un valor representacional que subraya su importancia como componente esencial de la vida humana, el diario no omite las consecuencias negativas que estos discursos tienen y han tenido para muchas mujeres. En cuanto al primer punto de vista, Shields corrobora que "I have never for one minute regarded the lives of women as trivial, and I've always known that men and women alike possess a domestic life that very seldom finds its way into our fiction" (Random Iluminatons 47). De ahí que la novela comience con la representación detallada de las tareas domésticas de su madre, Mercy, que identifican la domesticidad con una forma de creatividad. Los detalles de la preparación del pudding como si fuera una obra de arte y la pasión por el hogar, particularmente por la cocina como el espacio en el que realizarse, contribuyen a la exhibición de una imagen bastante positiva de la domesticidad desde el punto de vista femenino. Esto, tal como Shields mencionaba, ha estado tradicionalmente ausente en la literatura.

No obstante, la vecina, Mrs Flett, deja ver la otra cara de la domesticidad al mostrar el aislamiento de las mujeres dentro del hogar. La insatisfacción y la soledad de las amas de casa se plasma a través de la experiencia de Clarentine que se resume en la siguiente 
cita: "Women need companionship — that was the very thing Dr. Spears was fussing her about. Maybe that was all that was the matter with her, nothing but loneliness [...] She and Mercy are alone in the world, two solitary souls, side by side in their separate houses, locked up with the same circle of anxious hunger" (The Stone 19). Tanto Mercy como Mrs Flett pueden considerarse proyecciones de la propia narradora. De hecho, la construcción de Daisy de su madre lleva a límites extremos el prototipo de 'Mujer' dictado por el discurso dominante, creando un personaje que se estructura en torno a tres aspectos: su cuerpo, su silencio y la domesticidad. Mercy simboliza la visión más tradicional de la feminidad, el ama de casa perfecta, idealizada, cuyo cuerpo parece ocuparlo todo, pero que, paradójicamente, se encuentra ausente en la vida de su hija, pues muere al dar a luz.

$\mathrm{El}$ arquetipo de 'mujer' que Mercy personifica supone un exceso, casi un esperpento, pero es también un ideal que devuelve a Daisy el reflejo de su propia inadecuación, proyectado posteriormente en Mrs Flett. En relación con esto, la preparación del pudding de su madre al principio de la novela contrasta con la cena que Daisy le prepara a su marido en el capítulo "Motherhood", en el que se desprende una visión muy crítica de la domesticidad. La soledad, la frustración y los sentimientos de inadecuación de Daisy al compararse con el ideal de la mística suscitan una lectura especialmente crítica de la 'mística de la feminidad', cuyo epítome está representado por Mercy. Los mandatos sociales impuestos por el discurso de género, en este caso el de la domesticidad, limitan la libertad de elección de las mujeres, constituyendo en muchas ocasiones una fuente de insatisfacción e incluso de trastornos psicológicos. Por consiguiente, la representación del espacio doméstico en el diario no implica una idealización ni la aceptación sin más de la organización de los espacios según el género. $\mathrm{Al}$ contrario, numerosas huellas permiten vislumbrar la insatisfacción de Daisy tanto en su matrimonio como en lo que concierne a la vida doméstica.

De la representación de la domesticidad se traslucen las distintas consecuencias que la asignación de los espacios tiene para hombres y mujeres. Daisy y su madre se ven recluidas en el hogar, aisladas de otras mujeres, al tiempo que realizan un trabajo carente de reconocimiento social. El imperativo de la domesticidad circunscribe sus vidas a las cuatro paredes del hogar donde se les priva de un espacio y tiempo propios, con la consecuente pérdida de individualidad que esto supone. 
La domesticidad tiene el efecto adicional de contribuir a la construcción de un tipo de subjetividad femenina basada en la ética de los cuidados:

la domesticidad [...] es más un comportamiento, una disposición a prestar atención y dar respuesta a las necesidades del otro [...] hacerse cargo, por encima del propio interés, de lo que puedan necesitar o desear los demás [...] La domesticidad asignada a las mujeres conlleva la negación de un tiempo y espacio propios y por tanto la imposibilidad de construcción de la individualidad. (Beltrán y Maquieira 157)

La definición de una feminidad ligada a la domesticidad fomenta una identidad relacional. Si bien todas las vidas se encuentran inevitablemente implicadas en las historias de otros, Daisy deja al descubierto los peligros de la identidad relacional para las mujeres.

Aunque la identidad relacional no atañe únicamente a la femenina, sino que es común a la especie humana, tradicionalmente se ha concebido como una cualidad típicamente asociada a las mujeres. En este punto, conviene detenerse en la deconstrucción del ideal de hombre hecho a sí mismo, cuya madurez conlleva su individualización y separación del grupo en un proceso que se ha representado en los últimos siglos a través de la autobiografía tradicional masculina. La siguiente cita recoge los puntos principales de la narración del sujeto hegemónico: "the traditional development of the male autobiographical self begins in relationship (to a person, a family, a place) but develops into an understanding of his separateness from others, the nonidentical correspondence of relationship, the self-identical foundation of the proper name" (Gilmore 29). Frente a este modelo, The Stone Diaries ofrece una caracterización de la subjetividad que es inseparable de las relaciones con otros. En oposición al individualismo de las teorías liberales, la novela hace hincapié en la importancia de los vínculos interpersonales para la constitución del yo.

El diario problematiza su conexión con la construcción de la feminidad al demostrar cómo la individualidad de Daisy se ve sacrificada por sus relaciones con los demás. A través de ella se constata la amenaza que la identidad relacional presenta para las mujeres al incorporarlas a 'las idénticas' (Amorós 10). De ahí la ironía de que hacia el final de la novela Daisy se culpabilice por su egocentrismo, "always on and on about her own concerns. Instead of thinking of others. Putting other first" (The Stone 340). La cita reproduce el discurso dominante 
que obliga a las mujeres a anteponer el bienestar y las necesidades de otros a las suyas propias. Esta declaración, sin embargo, tiene efectos paródicos en el contexto de una auto/biografía que destaca por la ausencia de la narradora frente a la presencia y constante invasión de las voces de otros.

En el capítulo "Motherhood", Daisy se describe como el pilar que sostiene a la familia, la encargada de proporcionar los cuidados y afectos de los que sus hijos y su marido disfrutan. La contrariedad que esto reporta es que ella se define casi exclusivamente por sus conexiones dentro del ámbito privado como madre y esposa. En el primer capítulo se hizo alusión a las distintas denominaciones o, como ella misma expresa, "her life definitions", que la vinculan siempre a un otro: es la hija, la esposa, la mujer, la madre, la tía o la abuela de otros. Wendy Roy, quien repara en los diversos riesgos derivados de una subjetividad basada exclusivamente en las relaciones, concluye con la idea de que "if a woman can only represent herself in relation, she may, like Daisy, become lost in the interconnections that constitute her sense of self" (Roy 119).

La falta de individualidad de Daisy se reitera a lo largo del diario mediante distintas vías. Configurada exclusivamente a través de los roles que la unen a otros, la protagonista se asimila a una de estas 'idénticas' carentes de singularidad. Es muy interesante cómo durante la depresión de Daisy, su hijo Warren equipara la vida de las mujeres de esta extracción social a una trampa:

if I feel cheated, how much more cheated she must feel. She must be in mourning for the squandering of herself. Something, someone, cut off her head, yanked out her tongue. My mother is a middle-aged woman, a middle-class woman, a woman of moderate intelligence and medium-sized ego and average luck, so that you would expect her to land somewhere near the midde of the world. Instead she's over there at the edge. The least vibration could knock her off. (The Stone 252)

Puesto que Daisy es la narradora del diario, las palabras de Warren también se le deben atribuir a ella. De esta forma, el fragmento señala que la protagonista es plenamente consciente del engaño al que se ha visto sometida, pues si bien a las mujeres se les adjudican unos espacios, roles y mandatos sociales diferentes a los de los hombres, el sistema de valores que comparten es idéntico. 
La realización personal a través de los roles de madre y esposa dictados por "la mística de la feminidad" se contrapone con los ideales de una cultura que además de atribuir mayor reconocimiento a la esfera pública dominada por los hombres, basa su modelo del 'sueño americano' en la riqueza y el poder logrado en este dominio. En otras palabras, las mujeres participan en un sistema de valores que da mayor prestigio a las actividades asociadas a los hombres. La cita de Warren también hace referencia a los ensayos que Daisy escribió durante sus años en la universidad, lo que pone de manifiesto la contradicción que entraña dotar a las mujeres de una formación académica y de unas ideas sobre el desarrollo personal para posteriormente relegarlas a tareas domésticas carentes de prestigio social. La trampa a la que hacía referencia Warren consiste en que a pesar de disfrutar de una condición social privilegiada en tanto mujer blanca de clase media en América, Daisy se encuentra en los márgenes debido a su género.

$\mathrm{El}$ acatamiento de Daisy de las normas y roles de género converge con una voz crítica que cuestiona los discursos que dominan su desarrollo vital: "knowledge that here, this place, was where she would continue to live her life, where she had, in fact, always live -blinded, throttled, erased from the record of her own existence" (The Stone 76). A través de su narradora, Carol Shields no sólo trata de llenar el vacío que rodea la vida de mujeres como Daisy, sino que también pone por escrito los discursos de género que han articulado la identidad femenina del sector de mujeres blancas, de clase media durante gran parte del siglo XX en Estados Unidos. De esta forma, da voz a un silencio que lejos de dar paso a una visión complaciente, traza una crítica sobre la que se erige la visión del feminismo propuesta por la autora.

A pesar de la influencia de la mística de la feminidad, la construcción de una subjetividad femenina relacional y fragmentaria contrasta con las teorías liberales lideradas por Betty Friedan en The Feminine Mystique, lo que indica que Shields no acepta ciegamente los presupuestos de este feminismo. Además, el análisis de la domesticidad de la protagonista delata el sesgo de clase que impide generalizar la experiencia de Daisy a mujeres pertenecientes a otras clases sociales. En Feminist theory: from margin to center, bell hooks critica la insensibilidad de Betty Friedan y otras feministas de la segunda ola hacia las diferencias de clase, raza, etnia o sexualidad que separan a las mujeres, alegando que aunque la situación de las burguesas merezca atención pública, 
sus problemas no son universales ni son más graves o urgentes que la discriminación múltiple que afecta a muchas otras mujeres. hooks dirige a Friedan reproches como el de que "[s]he made her plight and the plight of white women like herself synonymous with a condition affecting all American women. In so doing, she deflected attention away from her classism, her racism, her sexist attitudes towards the masses of American women" (2). Masas de mujeres, añade, cuya mayor preocupación era la supervivencia económica o la discriminación étnica y racial. The Stone Diaries, por el contrario, incide en la especificidad de Daisy, particularmente en la variable de clase.

La representación del yo de Daisy ofrece una visión de la subjetividad que cuestiona algunos de los principales presupuestos tanto del feminismo liberal como del feminismo de la diferencia, en tanto su fragmentación impide trazar cualquier clase de conclusión acerca de la identidad femenina. Carol Shields resume magníficamente este último punto cuando define el diario como "a search for meaning or authenticity and it isn't found" (53). Desde la perspectiva de "a middleclass woman, a woman of moderate intelligence and medium-sized ego and average look" (The Stone 252), The Stone Diaries narra una vida desplazada por las historias de otros, una subjetividad descentrada y fragmentada por los discursos sociales que la atraviesan y un yo cuya individualidad se ve mermada por las relaciones que establece.

La inscripción literaria lleva implícita la subversión, tanto del modelo de feminidad instituido como de la construcción de la identidad canónica. La política de representación desde la que Daisy, en palabras de Sidonie Smith, "speaks to her culture from the margins" (180) presupone una política de localización que deconstruye la subjetividad universal y hegemónica. Asimismo, Carol Shields combina la crítica feminista con la decontrucción postmoderna de la representación del sujeto. Como a continuación se concluye: "In this transformative resistance, The Stone Diaries, in all its partiality and glorious indecision, suggests that women have the capacity to narrate ourselves into a particular way of being in the world, absorbing our surroundings through touch, taste, and personal testimony, then enlarging on the available materials" (Johnson 224).

Por otra parte, la clasificación de la novela como una meta/ autobiografía saca a la luz la visión de la autora sobre determinados 
debates literarios que la vinculan expresamente con el postmodernismo. La metaficción, la fragmentación del yo, el énfasis en los discursos que atraviesan al sujeto, la muerte del autor que impregna capítulos como el de "Sorrow, 1965" o la puesta en práctica de técnicas consideradas típicamente postmodernas plasman la influencia del postmodernismo. Sin embargo, la intersección con la representación de las condiciones materiales de las mujeres y la crítica feminista subyacente a The Stone Diaries proporcionan a la novela una función política vinculada al feminismo. La combinación de todos estos aspectos confluye en un feminismo postmoderno. Shields desarrolla una teoría feminista en la que el feminismo y el postmodernismo no sólo coexisten, sino que se complementan: "for some feminists, postmodernism is not only a natural ally but also provides a basis for avoiding the tendency to construct theory that generalizes from the experience of Western, White, middleclass women" (Arriving Late 5).

Tanto dentro como fuera de la novela, el feminismo y el postmodernismo convergen en muchos de sus fundamentos. La declaración postmoderna de la muerte del sujeto profundizó en la deconstrucción de los ideales de la Ilustración, contribuyendo así a la articulación de otras voces. En The Stone Diaries se celebra la apertura de nuevas posibilidades que emerge del colapso de las estructuras. Un ejemplo de ello se observa en la siguiente cita: "The synapses colapse; well, let them. She enlarges on the available material, extends, shrinks, reshapes what's offered; this mixed position is her life. She swirls it one way or the other, depending on -who knows what it depends on?the fulcrum of desire, or of necessity" (The Stone 282). Esta correlación entre el colapso de las estructuras de pensamiento y la expansión de la realidad proyectada a lo largo del diario de Daisy es análoga al auge de nuevos canales de expresión y de voces alternativas facilitadas por la crisis postmoderna.

Linda Johnson discierne una simbiosis entre el feminismo y el postmodernismo dentro de la novela cuando afirma que "Shields employs common postmodern aesthetic strategies -the fragmented narrator, hybrid genre, and metafictional narrative for example- but she joins them with a thematic insistence on the transformative female imagination [...] enacting an embodied, woman-centred, and politicized postmodernism" (203). Mediante la ficción auto/biográfica The Stone Diaries ilustra y demuestra que la confluencia entre el feminismo y 
el posmodernismo subsana y completa ambos movimientos al superar el punto muerto entre las tendencias universalizadoras del feminismo y las críticas al postmodernismo por su falta de compromiso político. En este caso, la problemática postmoderna de la representación del yo se vincula también con la crítica feminista sobre los discursos de la representación de subjetividades femeninas. Esto tiene importantes consecuencias para la política del texto, en tanto la novela orienta el postmodernismo hacia las condiciones materiales de la vida de las mujeres, contribuyendo a un postmodernismo anclado en la especificidad del cuerpo.

La fructífera alianza entre feminismo y postmodernismo contribuye a una representación ficticia que tiene la capacidad de actuar sobre la realidad para desempeñar una función política. Asimismo, la auto-reflexividad de la novela desvela las limitaciones inherentes al lenguaje, así como las oportunidades que ofrece a quien escribe a la hora de intervenir en el mundo. Aparte de las reflexiones sobre la intersección entre la memoria y la imaginación o la ficción y la realidad en la escritura autobiográfica, Carol Shields formula su visión de la ficción y su potencialidad de influir sobre la realidad en su ensayo "Narrative hunger and the overwhelming cupboard". En él atribuye a la literatura un poder sobre el mundo exterior a la obra, al tiempo que reconoce el valor ético de ahondar en este vínculo: "For if literature is not about the world, what is it about? Luckily all the world is up for sale. Unluckily, a good part of the world falls through the narrative sieve, washing through the fingers of the recorders' hands and becoming lost. It is this simultaneous abundance and loss that I want to think about" (Narrative Hunger 20).

Puesto que Carol Shields utiliza la ficción no sólo para reflexionar sobre la escritura, sino también con el fin de intervenir en su contexto, los distintos niveles de lectura desplegados en la novela llevan a considerar sus dos papeles como escritora y crítica. Esto se apoya en la idea de Martin de que cuando una escritora "talks about a narrative within that narrative [...] has become a theorist" (39). A través de esta investigación se pretende incorporar a Carol Shields dentro del grupo de escritoras canadienses contemporáneas que han luchado por poner en entredicho la tradición establecida mediante la propuesta de una alternativa capaz de integrar la política feminista con algunos elementos de la condición postmoderna. La inclinación de 
Shields por los asuntos relativos al ámbito femenino, incluido el espacio doméstico, explican que durante años se infravalorase su trabajo. Ella misma menciona la tendencia a subestimar la literatura femenina: "I see women's books demeaned because they deal with the material of women's lives" (Wachtel 26). De hecho, el interés fundamental de The Stone Diaries radica en la representación de la subjetividad y la vida de mujeres. A la vez que ofrece una crítica, la novela representa una visión alternativa que aspira, en última instancia, a transformar tanto la vida como la escritura de las mujeres.

Del estudio de la política de representación de Daisy se ha pasado a la función política del feminismo postmoderno subyacente a la obra de Shields. La representación de la vida y la escritura de las mujeres forman los ejes centrales de The Stone Diaries. Consciente del silencio y la marginalización que la tradición patriarcal ha impuesto sobre ambas, la autora se sirve de la ficción para denunciar este vacío, dotándolo a su vez de una voz. Con este objetivo en mente crea a Daisy, de quien explica "I intend her to be evasive, although any woman in this century can understand what it feels like to be erased from the culture" (Thomas 60). Shields ilustra y denuncia el silencio histórico de las mujeres a través de la ausencia de cartas o cualquier documento que represente directamente la voz de Daisy, así como las menciones a las pérdidas de distintos escritos, como sus cartas a Barker o su diario de viaje. Es más, su decisión de dejar de escribir un diario tras casarse sugiere una incompatibilidad entre el papel de las mujeres dentro de la familia y la búsqueda de subjetividad que conlleva la escritura, pues el modelo de familia burguesa patriarcal se apoya en una figura materna que sacrifica su individualidad y su felicidad por el bienestar de los suyos. La siguiente cita llama la atención sobre dos de las causas que han contribuido a la pérdida de historias femeninas; la primera se debe a factores materiales, concretamente a la desaparición de un diario, mientras que en el segundo caso se trata de unas memorias que nunca llegaron a escribirse: "Daisy Goodwill's own thoughts on her marriage are not recorded, for she has given up the practice of keeping a private journal. The recent loss of her travel diary -it has never been foundcaused her a certain amount of secret grief" (The Stone 156).

Dentro de la novela Daisy compagina el propósito aparentemente contradictorio de dejar patente la ausencia representada por la subyugación femenina con la recuperación del dominio sobre su 
vida mediante la imaginación de pasados alternativos. Su diario se concibe como testimonio del silencio de las mujeres, pero es también un monumento "to the movement of possible music" (The Stone 2). De forma análoga, Carol Shields se ampara en la ficción motivada por esta doble intención, la de denunciar un silencio y ofrecer una alternativa imaginaria por medio de la literatura. Con esto se plantea una cuestión fundamental que Leigh Gilmore define en Autobiographics de la siguiente forma: "how do we re/member [...] which I take to imply both the act of memory and the restoration of erased persons and texts as bodies of evidence" (27). Por lo tanto, a la hora de analizar la obra de Shields es necesario profundizar en estas dos vertientes. En relación con esto, ya se mencionó cómo en "Narrative Hunger and the Overflowing Cupboard" presenta su propia teoría sobre la lectura y la escritura, identificando toda la realidad como un sinfín de historias en potencia.

Las condiciones materiales pueden contribuir a que muchas de las versiones posibles en un contexto dado no logren sobrevivir en el tiempo o, simplemente, nunca lleguen a articularse. La pérdida incalculable de historias de mujeres ha sido consecuencia en muchos casos de condiciones materiales y discursivas que durante años se encargaron de silenciarlas: "Enormous quantities of stories -perhaps the finest stories of our culture- have been lost to illiteracy or lack of permission, either a prohibition placed on the storyteller -most often: 'Woman, hold thy tongue'- or the simple inability to write down one's experience on paper" (Narrative Hunger 26). Las palabras de Shields apuntan a la acción conjunta de las estructuras materiales y simbólicas que supuso que ciertas voces se vieran silenciadas, determinados aspectos de la experiencia humana permanecieran ocultos, y la representación parcial y subjetiva del grupo dominante pasase a considerarse como universal. El resultado, por tanto, ha sido la omisión de distintas perspectivas, interpretaciones y aproximaciones a la realidad.

Asimismo, numerosas investigaciones sobre las auto/biografías femeninas señalan que el problema no radica únicamente en la escasez de escritos de mujeres, sino en la valoración que la crítica, mayoritariamente masculina, le ha dado a estos textos al leerlos según los parámetros de la tradición patriarcal hegemónica. Puesto que no se trata de una cuestión de producción, sino de poder, la escritura auto/ biográfica de ficción constituye un acto político al denunciar un silencio histórico que en muchos casos se identifica no tanto con un problema 
de presencia como de invisibilidad. La ausencia de la protagonista de una auto/biografía que insiste en su condición de construcción histórica permite interrogar los criterios por los que la escritura de las vidas femeninas se han considerado insustanciales e insignificantes. "What Shields offers is what Carolyn Steedman has described in another context as 'an altered sense of the historical meaning and importance of female insignificance [...] A sense of that which is lost, never to be recovered completely" (107). Aún más importante, el reconocimiento de una pérdida irreparable en el pasado se contrarresta gracias a la ficción. $\mathrm{Al}$ ofrecer una versión alternativa de la temporalidad y espacialidad en la que se despliega el pasado de Daisy, The Stone Diaries aboga por la imaginación como amparo frente al pasado, y la ficción frente a una historia que acusa de androcentrismo o "his/story". De esta forma, se destaca el poder de la ficción de recuperar aquello que la historia ha silenciado o, volviendo a la frase de Ricoeur, el potencial de "liberar retrospectivamente ciertas posibilidades no efectuadas del pasado histórico [...] El cuasi pasado de la ficción se convierte así en el revelador de los posibles escondidos en el pasado efectivo" (916).

Lo que Shields define en una de las citas anteriores como "postmodern box-within-the-box" es también aplicable al encadenamiento entre la manipulación de Carol Shields de la ficción para exponer y subsanar el silencio histórico de las mujeres y la utilización por parte de Daisy de la imaginación para llenar las lagunas de su pasado. Shields es consciente de que ciertos aspectos pertenecientes a la privacidad y al dominio más íntimo de las vidas humanas sólo pueden articularse y transmitirse mediante la ficción, y en base a este reconocimiento elabora su novela:

It is through fiction that I've learnt about the lives of women. And about how people think; biography and history have a narrative structure, but they don't tell us much about the interior lives of people. This seems to me to be fiction's magic, that it attempts to be an account of all that cannot be documented, but is, nevertheless, true. (cit. en Roy 138)

La imaginación y, por extensión, la literatura no sólo permiten recuperar las historias de mujeres olvidadas sino también retratarlas de manera significativa, otorgándoles un valor representacional que de algún modo repare los abusos y parcialidades de la historia. Es decir, la literatura representa el canal mediante el que elaborar las 'contramemorias'. Alex Ramon, quien también atribuye esta función a 
la obra, la define como "fundamentally counter-historical, offering the opportunity for a minute examination of the life and voices that `slip through the net of history' and an excavation of its apparent 'empty cavities" (127).

Shields se sirve del papel redentor de la literatura de recuperar aquello que de otra forma habría quedado relegado al silencio: "I have this impulse to see fiction as a form of redemption, to redeem what otherwise might be lost" (2007: 52). La paradoja última de la novela radica en que la redención, cuya condición es el reconocimieto en el espacio público de narrativas que necesariamente presuponen una presencia, se lleva a cabo mediante la exposición de una ausencia. En conclusión, el postmodernismo de Shields combina la política de la contramemoria femenina con la deconstrucción postestructuralista. Lejos de ofrecer una narración desde el punto de vista de un sujeto femenino unificado o universal, The Stone Diaries opta por una narradora carente de cualquier "kernel of authenticity, that precious interior core that everyone around her seem[s] to possess" (The Stone 75). Daisy se encuentra fragmentada por una serie de discursos que la desplazan del centro narrativo que continuamente se ve invadido por las perspectivas e historias de otros. Esto le permite denunciar la situación de desigualdad de Daisy sin recurrir a esencialismos ni a generalizaciones de la experiencia de un grupo privilegiado de mujeres sobre el resto.

\section{WORKS CITED}

Amorós, Celia. Feminismo y Filosofía. Madrid: Síntesis, 2000.

Beltrán, Elena y Virginia Maquieira. Feminismos. Debates Contemporáneos. Madrid: Alianza Editorial, 2001.

Eakin, Paul. How Our Lives Become Stories: Making Selves. Ithaca: Cornell University Press, 1999.

---. Eakin, Paul. Living Autobiographically. How we Create Identity in Narrative. New York: Cornell University Press, 2008.

Friedan, Betty. The Feminine Mystique. Harmondsworth: Pelican Books, 1982. 
Gilmore, Leigh. Autobiographics: A Feminist Theory of Women's SelfRepresentation. Ithaca: Cornell University Press, 1994.

hooks, bell. Feminist Theory: From Margin to Center. Boston: South End Press, 1984.

Johnson, Lisa. "She Enlarges on the Available Materials:' A Postmodernism of Resistance in The Stone Diaries." En Carol Shields, Narrative Hunger, and the Possibilities of Fiction, Edward Eden \& dee Goertz. Toronto: University of Toronto, (2003): 129-201.

Martin, Wallace . Recent Theories of Narrative. Ithaca: Cornell University Press, 1986.

Nicholson, Linda. Feminism / Postmodernism. New York: Routledge, 1990.

Ramon, Alex. Liminal Spaces: The Double Art of Carol Sheilds. Newcastle upon Tyne: Cambridge Scholars, 2008.

Ricoeur, Paul. Tiempo y Narración. Buenos Aires: siglo XXI, 1995.

Roy, Wendy. "Autobiography as Critical Practice in The Stone Diaries." En Carol Shields, Narrative Hunger, and the Possibilities of Fiction, Edward Eden \& dee Goertz, Toronto: University of Toronto, (2003): 113-146.

Shields, Carol. “The Golden Book:' An Interview with Carol Shields.” Prairie Fire 14.4. (1993): 56-62.

---. “Arriving Late: Starting Over." En How Stories Mean, John Metcalf \& J. R. Struthers. Erin: Porcupine's Quill, (1993): 244-251.

---. The Stone Diaries. New York: Penguin Books, 1993.

---. "Narrative Hunger and the Overflowing Cupboard." En Carol Shields, Narrative Hunger, and the Possibilities of Fiction, Edward Eden \& dee Goertz, Toronto: University of Toronto, (2003): 19-36.

---. Random Illuminations: conversations with Carol Shields. En Eleanor Wachtel. New Brunswick: Goose Lane Editions, 2003.

Smith, Sidonie \& Julia Watson. De / colonizing the subject: The politics of gender in women's autobiography. Minneapolis: University of Minnesota Press, 1992.

Steedman, Carolyn. "Women's Biography and Autobiography: Forms of History, Histories of Form". En From My Guy to Sci-Fi: Genre and Women's Writing in the Postmodern World, Helen Carr. London: Pandora Press, (1989): 98-111.

Thomas, Joan (1993). “The Golden Book': An Interview with Carol Shields.” Prairie Fire, (1993): 56-62.

Wachtel, Eleanor. "Interview with Carol Shields." Room of One's Own: A Feminist Journal of Literature and Criticism 13, (1989): 5-45.

Received: 01 September 2014

Accepted: 31 October 2014 


\title{
UN CASO NOTABLE DE TRADUCCIÓN INDIRECTA: THE LAST OF THE MOHICANS DE FENIMORE COOPER TRADUCIDO POR VICENTE PAGASARTUNDUA A TRAVÉS DEL FRANCÉS
}

\author{
Ángeles García Calderón \\ Beatriz Martínez Ojeda \\ Universidad de Córdoba
}

\begin{abstract}
In the world of translation, the translator feels sometimes identified with the writer, to the extent that he often achieves a real mimesis with him. Needless to say, this relationship seems apparent between a novelist and a translator. It is surprising, however, when it takes place between the translator of a translator and the original he is translating (a translation within another translation). This is the case of the Spaniard Pagasartundua, when he translates an English novel through the French translation of Defauconprêt. Whereas JeanBaptiste Defauconprêt is recognized by the critics as the 'mediator' in France between Walter Scott and Fenimore Cooper, Pagasanturdua was the one who made the American novelist known to the Spanish readers from the vantage point of the prestigious French translator.
\end{abstract}

Key words: American Novel, Indirect Translation, Fenimore Cooper, Defauconprêt, Pagasartundua.

\section{Resumen}

En el mundo de la traducción la figura del traductor se identifica de tal modo con la del autor del texto original que alcanza a veces un mimetismo absoluto con él. Huelga decir que la relación parece lógica entre el novelista y el traductor, pero es más sorprendente cuando ocurre entre un 'versionista' del traductor y el original que traduce; éste es el 
caso del español Pagasartundua, que versiona una novela americana a través de la traducción francesa de Defauconprêt. Si Jean-Baptiste Defauconprêt es reconocido por la crítica como el 'intermediario' en Francia de Walter Scott y Fenimore Cooper, Pagasartundua será quien dé a conocer al novelista americano a los lectores españoles a través del filtro del prestigioso traductor francés.

Palabras clave: Novela americana, versión indirecta, Fenimore Cooper, Defauconprêt, Pagasartundua.

\section{Introducción: Los comienzos de la literatura estadounidense}

La literatura americana tuvo desde un primer instante todos los rasgos y características inherentes a las de un nuevo país, ya que ninguna colonia producía literatura original, sino que generalmente se limitaba a proporcionar cuadros exóticos a los novelistas de evasión y a los defensores del imperialismo. Desde el momento en que se inicia cualquier colonización suele transcurrir bastante tiempo hasta que llega la independencia y esta empieza a verse como algo común. En América, hasta finales del XVIII, aparecieron algunos escritores notables, aunque ello no llegara a constituir en modo alguno una literatura, ya que entre la Declaración de la Independencia (1776) y la publicación de las primeras obras auténticamente originales transcurriría como mínimo medio siglo. ${ }^{1}$

La aparición de estas obras no parecía responder totalmente al pensamiento de la crítica inglesa sobre la nueva nación, siendo reveladora a este respecto la opinión del clérigo anglicano, escritor y crítico de la prestigiosa Edinburgh Review, Sydney Smith, el cual defendía que los norteamericanos no tuvieran necesidad alguna de cultivar una literatura propia, si en su propia lengua podían aprovecharse del sentido común inglés, así como de la ciencia y del genio de esa nación. Según él, las praderas, los barcos de vapor y las fábricas

\footnotetext{
${ }^{1}$ Podemos incluir en este apartado The Sketch Book of Geoffrey Crayon, Gent de Washington Irving (colección de treinta y cuatro ensayos y "short stories") publicados entre 1819-20, la novela histórica de Fenimore Cooper: The Pioneers: The Sources of the Susquehanna; a Descriptive Tale (1823); y el primer volumen de poemas de Poe, publicado anónimamente: Tamerlane and Other Poems (1827).
} 
de harina serían los únicos elementos naturales que se ofrecerían a la mirada de los colonos durante los siglos venideros. ${ }^{2}$

\section{James Fenimore Cooper: The Last of the Mohicans (1826)}

El más representativo de los escritores norteamericanos, notable por su tratamiento de las novelas de las praderas es James Fenimore Cooper (1789-1851). Nacido en New Jersey, pasó parte de su juventud en la hacienda familiar de Cooperstown en Otsego Lake (New York), parte en la marina mercante (tras ser expulsado de la Yale University), y parte en la marina americana, hasta 1811. Tras casarse, se instaló en el condado de Westchester. De 1826 a 1833 viajaría por Europa. $\mathrm{Su}$ primera novela, Precaution, ${ }^{3}$ pasaría inadvertida; pero un año más tarde The Spy (1821), una emocionante historia de la revolución americana lo haría famoso. The Pioneers (1823) sería la primera de su conocido grupo de novelas Leathers-Stocking Tales, conocidas luego por el nombre de su protagonista, el pionero explorador Natty Bumppo (alias 'Deerslayer', 'Pathfinder', o 'Hawkeye'). La serie continuaría con The Last of the Mohicans (1826), The Prairie (1827), The Pathfinder (1840) y The Deer-slayer (1841). Los relatos trataban de las aventuras de la frontera y proporcionaban una brillante descripción de la vida de los indios americanos y de los colonizadores, concediendo a Cooper la reputación de ser el "American Walter Scott". A su vuelta de Europa publicaría varios "critical accounts" sobre la sociedad europea, incluyendo entre ellos: England, with Sketches of Society in the Metropolis (1837), que fue violentamente atacado en Inglaterra, sobre todo por John Lockhart. Cooper sería también muy crítico con la

\footnotetext{
${ }^{2}$ Opinión incluida en la sección de artículos titulada "America", en The Works of the Rev. Sydney Smith, (Taylor 93-101; 137-141).

Un estado de la cuestión bastante exacto, que nos ha servido para este punto, podemos encontrarlo en los siguientes enlaces:

http://www.unizar.es/departamentos/filologia_inglesa/garciala/z13-12.html html

http://vanityfea.blogspot.com.es/2013/12/la-literatura-norteamericana-hasta-1800.

${ }^{3} \mathrm{La}$ "Novel of Manners" era un subgénero de la novela realista, cuyos precedentes serían Henry Fielding (1707-1754) y Samuel Richardson (1689-1761). Tras ellos, se puede afirmar que se convertiría en un subgénero femenino, cultivado por Charlotte Smith (1749-1806), Elizabeth Inchbald (1753-1821), Frances Burney (1752-1840), Jane Austen (1775-1817) Frances Trollope (1780-1863), Susan Ferrier (1782-1854), Catherine Gore (1799-1861) y Harriet Martineau (1802-1876). Sus características más importantes eran la vida cotidiana, el matrimonio como institución, el comportamiento en sociedad, etc.
} 
democracia americana, expresando sus opiniones conservadoras en el libro: The American Democrat (1838). Mark Twain humorísticamente atacaría su falta de precisión verbal en el ensayo Fenimore Cooper's Literary Offences (1895).

Sus novelas pasarían del círculo de lectores adultos al de los jóvenes, y de éstos al de los autores olvidados, aunque se sigan estudiando en los cursos de literatura de Estados Unidos. Cooper era hijo del fundador de una ciudad que sería centro de un nuevo condado, Coopersville, y había recorrido en su infancia la pradera así como los mares a bordo de navíos de toda clase. En sus relatos Cooper describe todo lo que ha vivido y padecido, siendo el primero de los escritores americanos que intuirá la sobrehumana grandeza de su país, trasladándolo todo a una epopeya que no debe casi nada a Europa. La paradoja o contradicción que se debate en su conciencia estriba entre la simpatía hacia los indios y el hecho de pertenecer al pueblo que los extermina, aunque esto no sea motivo para no retratar en su héroe al hombre que simboliza la aurora de la nueva nación.

El libro en cuestión sería traducido al español como El último mohicano, o también como El último de los mohicanos, ${ }^{4} \mathrm{y}$ su historia se desarrolla en el año 1757, durante la Guerra franco-india, ${ }^{5}$ en la época en que Francia y Gran Bretaña combaten por el control de las colonias de Norteamérica.

Teniendo en cuenta el número total de ediciones de sus obras en español, ocupa claramente el primer lugar por delante de escritores como Edgar Allan Poe o Walt Whitman, por indicar únicamente algunos de los más próximos a él en el tiempo. ${ }^{6}$

${ }^{4}$ Además de la traducción de Pasagartundua existe otra del mismo año: El último mohicano. Historia americana escrita en inglés por J. F. Cooper, Valencia: Imprenta de J. de Orga, 1832. En su conocida obra sobre la novela española del XIX Montesinos anota 4 volúmenes, aunque en realidad sean sólo tres (Montesinos 1982 176).

${ }^{5}$ La Guerra franco-india (1754-1763) fue una parte de la Guerra de los Siete Años (1756-1763) entre británicos, franceses y distintas tribus de los indios americanos.

${ }^{6}$ Un detallado estado de esta cuestión, así como de las traducciones al español de las obras de Fenimore Cooper, lo podemos hallar en el trabajo de Urbano Viñuela Angulo: "James Fenimore Cooper: Entre la popularidad y la transformación textual", Livius, 4, 1993. 


\section{La traducción francesa}

\subsection{El traductor: Auguste-Jean-Baptiste Defauconprêt (1767-1843)}

Junto a Jean Cohen, La Place, Constant de Rebecque, Isabelle de Montolieu, Mme Dubergier, Mme Molé de Champlâtreux y otros reputados traductores de la época, ${ }^{7}$ Defauconprêt fue un conocido escritor y traductor francés, que ganaría por su trabajo el "Prix d'honneur au concours général de l'Université" en 1786. Notario en París, se iría a vivir a Londres durante 25 años; allí llegó a adquirir un extraordinario dominio de la lengua inglesa que le permitiría llevar a cabo su ingente producción de traducción literaria con la colaboración de su hijo. ${ }^{8}$ De 1829 a 1864 publicaría más de cuatrocientos volúmenes de traducciones diversas de una gran altura, a pesar de la precipitación y rapidez de sus trabajos; del inglés traduciría las obras completas de Walter Scott (lo que ayudaría a difundir al novelista escocés por toda Europa) y también de James Fenimore Cooper, así como un gran número de obras de otros escritores, pertenecientes casi todos ellos a su propia generación: Ben Jonson, Henry Fielding, Laurence Sterne, Ann Radcliffe, Maria Edgeworth, Horace Smith, James Justinian Morier, Washington Irving, Thomas Gaspey, John Banim, Edward Bulwer-Lytton, Charles Dickens, Frederick Marryat, etc. Además de las traducciones de Scott y Cooper algunas de las versiones más conocidas de nuestro traductor son las siguientes: L'Orpheline du presbytère (de Anna Maria Bennett, 1816); Ormond (de Maria Edgeworth, 1817); Le Jeune Cleveland, ou, Traits de nature (de Sarah Harriet Burney, 1819); Florence Macarthy (de Lady Morgan, 1819); Le Mystère ou il y a quarante ans y Calthorpe (de Francis Lathom, 1821); Pen Owen (de James Hook, 1823); Sir André Wylie (1823), Les Lairds de Grippy y Annales de la paroisse (1824), Rothelan (las cuatro de John Galt); L'Héritage (de Susan Ferrier, 1824); Gaston de Blondeville (de Ann Radcliffe, 1826); Brambletye-House (de Horace Smith, 1826); L'Apostat ou la famille Nowland, L'AngloIrlandais, Crohoore Na Bilhoge (las tres obras de Banim, y de 1829).

\footnotetext{
${ }^{7}$ Una completa y extensa relación se encuentra en el libro Recueil de Préfaces de traducteurs de romans anglais 1721-1828.

${ }^{8}$ Charles Auguste (1797-1865), director de la École Sainte-Barbe.
} 


\subsection{Le Dernier des Mohicans (1826)}

La traducción de Defauconprêt se publicaría el mismo año que el original, en tres tomos, incluida en las obras completas de Fenimore Cooper trasladadas por nuestro traductor con la siguiente referencia: Le Dernier des Mohicans, histoire de 1757; par M. Cooper, traduite de l'anglais par M. Defauconprêt, traducteur de tous les romans historiques de Sir Walter Scott. Paris: Charles Gosselin, $1826 .{ }^{9}$

Defauconprêt, fiel en la traducción al texto original, incluye también el prólogo del texto original, el cual constituirá el objeto de nuestro análisis:

\section{PRÉFACE}

Le lecteur qui commence la lecture de ces volumes dans l'espoir d'y trouver le tableau romanesque et imaginaire de choses qui n'ont jamais existé l'abandonnera sans doute lorsqu'il se verra trompe dans son attente. L'ouvrage n'est autre chose que ce qu'annonce son titre, une relation..$^{10}$ Cependant, comme il renferme des détails qui pourraient n'être pas compris de tous les lecteurs et surtout des lectrices qu'il pourrait trouver, en passant pour une fiction, il est de l'intérêt de l'auteur d'éclaircir ce que les allusions historiques pourraient présenter d'obscur. Et c'est pour lui un devoir d'autant plus rigoureux qu'il a souvent fait la triste expérience que, lors même que le public ignorerait complètement les faits que vous allez lui raconter, dès l'instant que vous les soumettez à son tribunal redoutable, il se trouve individuellement et collectivement, par une espèce d'intention inexplicable, en savoir beaucoup plus que l'auteur lui-même. Ce fait est incontestable; eh bien, cependant qu'un écrivain se hasarde à donner à l'imagination des autres la carrière qu'il n'aurait dû donner qu'à la sienne, par une contradiction nouvelle, il aura presque toujours à s'en repentir. Tout ce qui peut être explique doit donc l'être avec soin, au risque de mécontenter cette classe de lecteurs qui trouvent d'autant plus de plaisir à parcourir un ouvrage qu'il leur

${ }^{9}$ Era de todo punto lógico que Defauconprêt sintiera la misma simpatía por Scott que por Cooper, el "American Scott español", como casi lo denomina Lawrence H. Klibbe en su trabajo "La visión de España en la novela de Cooper, Mercedes of Castile", Actas del X Congreso de la Asociación internacional de Hispanistas, Barcelona 21-28 de agosto de 1989, coord. por Antonio Vilanova, vol. 2, 1992.

${ }^{10}$ A Narrative. 
offre plus d'énigmes à deviner ou plus de mystères à éclaircir C'est par l'exposé préliminaire des raisons qui l'obligent dès le début à employer tant de mots intelligibles que l'auteur commencera la tâche qu'il s'est imposée. Il ne dira rien que ne sache déjà celui qui serait versé le moins du monde dans la connaissance des antiquités indiennes.

La plus grande difficulté contre laquelle ait à lutter quiconque veut étudier l'histoire des sauvages indiens, c'est la confusion qui règne dans les noms. Si l'on réfléchit que les Hollandais, les Anglais et les Français, en leur qualité de conquérants, se sont permis tour à tour de grandes libertés sous ce rapport; que les naturels eux-mêmes parlent non seulement différentes langues, et même des dialectes de ces mêmes langues, mais qu'ils aiment en outre à multiplier les dénominations, cette confusion causera moins de surprise que de regret; elle pourra servir d'excuse pour ce qui paraîtrait obscur dans cet ouvrage, quels que soient d'ailleurs les défauts qu'on puisse lui reprocher.

Les Européens trouvèrent cette région immense qui s'étend entre le Penobscot et le Potomac, l'Océan Atlantique et le Mississipi, en la possession d'un peuple qui n'avait qu'une seule et même origine. Il est possible que sur un ou deux points les limites de ce vaste territoire aient été étendues ou restreintes par les nations environnantes; mais telles en étaient du moins les bornes naturelles et ordinaires. Ce peuple avait le nom générique de Wapanachki; mais il affectionnait celui de Lenni Lenape, qu'il s'était donné, et qui signifie «un peuple sans mélange.» Lauteur avoue franchement que ses connaissances ne sont pas jusqu'a pouvoir énumérer les Communautés ou tribus dans lesquelles cette race d'hommes s'est subdivisée. Chaque tribu avait son nom, ses chefs, son territoire particulier pour la chasse, et même son dialecte. Comme les princes féodaux de l'ancien temps, ces peuples se battaient entre eux, et exerçaient la plupart des privilèges de la souveraineté; mais ils n'en reconnaissaient pas moins une origine commune; leur langue était la même, ainsi que leurs traditions qui se transmettaient avec une fidélité, surprenante. Une branche de ce peuple nombreux occupait les bords d'un beau fleuve connu sous le nom de «Lenapewihittuck.» C'était là que d'un consentement unanime était établie «la Maison Longue»ou «le Feu du Grand Conseil» ${ }^{11}$ de la nation.

\footnotetext{
${ }^{11}$ Great council fire.
} 
La tribu possédant la contrée que forme à présent la partie sudouest de la Nouvelle-Angleterre, et cette portion de New-York qui est à l'est de la baie d'Hudson, ainsi qu'une grands étendue de pays qui se prolongée encore plus vers le sud, était un peuple puissant appelé «les Mohicanni», ou plus ordinairement «les Mohicans.» C'est de ce dernier mot que les Anglais ont fait depuis par corruption «Mohégans.».

Les Mohicans étaient encore subdivisés en peuplades. Collectivement, ils le disputoient, sous le rapport de l'antiquité, même à leurs voisins qui possédaient la «Maison Longue;» mais on leur accordait sans contestation d'être «le fils aîné de leur grand-père.» Cette portion des propriétaires primitifs du sol fut la première dépossédée par les blancs. Le petit nombre qui en reste encore sont dispersés parmi les autres tribus, et il ne leur reste de leur grandeur et de leur puissance que de tristes souvenirs. La tribu qui gardait l'enceinte sacrée de la maison du conseil fut distinguée pendant longtemps par le titre flatteur de «Lenape;» mais lorsque les Anglais eurent change le nom du fleuve en celui de «Delaware,» ce nouveau nom devint insensiblement celui des habitants. En général ils montrent beaucoup de délicatesse et de discernement dans l'emploi des dénominations. Des nuances expressives donnent plus de clarté à leurs idées, et communiquent souvent une grande énergie à leurs discours.

Dans un espace de plusieurs centaines de milles le long des frontières septentrionales de la tribu des Lenapes, habitait un autre peuple qui offrait les mêmes subdivisions, la même origine, le même langage. Ces sauvages du nord étaient d'abord moins puissants et moins unis entre eux que les Lenapes. Afin de remédier cette désavantage, cinq de leurs tribus les plus nombreuses et les plus guerrières qui se trouvaient le plus près de maison du conseil de leurs ennemis se liguèrent ensemble pour se défendre mutuellement; et ce sont, par le fait, les plus anciennes Républiques Unies dont l'histoire de l'Amérique septentrionale offre quelque trace. Ces tribus étaient les Mohawks, les Onéidas, les Senecas, les Cayugas et les Onondagas. Par la suite une tribu vagabonde de la même, race, qui s'était avancée plus près du soleil, vint se joindre à eux, et fut admise à participer à tous leurs privilèges politiques. Cette tribu (les Tuscaroras) augmenta tellement leur nombre que les Anglais changèrent le nom qu'ils avaient donné à la confédération, et ils ne les appelèrent plus « les Cinq,» mais «les Six Nations.» On verra dans le cours de cette relation que le mot nation s'applique tantôt à une tribu 
et tantôt au peuple entier, dans son acception la plus étendue. Les Mengwes étaient souvent appelés par les Indiens leurs voisins Maquas, et souvent même, par forme de dérision, Mingos. Les Français leur donnèrent le nom d'Iroquois, par corruption sans doute de quelqu'une des dénominations qu'ils prenaient.

Une tradition authentique a conservé le détail des moyens peu honorables que les Hollandais d'un côté, et les Mengwes de l'autre, employèrent pour déterminer les Lenapes à déposer les armes, à confier entièrement aux derniers le soin de leur défense, en un mot à n'être plus, dans le langage figuré des naturels, que des femmes. Si la politique suivie par les Hollandais était peu généreuse, elle était du moins sans danger. C'est de ce moment que date la chute de la plus grande et de la plus civilisée des nations indiennes qui occupaient l'emplacement actuel des États-Unis. Dépouillés par les blancs, opprimés et massacrés par les sauvages, ces malheureux continuèrent encore quelque temps à errer autour de leur maison du conseil puis, se séparant par bandes, ils allèrent se réfugier dans les vastes solitudes qui se prolongent à l'occident. Semblable à la clarté de la lampe qui s'éteint, leur gloire ne brilla jamais avec plus d'éclat qu'au moment où ils allaient être anéantis.

On pourrait donner encore d'autres détails sur ce peuple intéressant, surtout sur la partie la plus récente de son histoire; mais l'auteur ne les croit pas nécessaires au plan de cet ouvrage. La mort du pieu et vénérable Heckewelder est, sous ce rapport, une perte qui ne sera peutêtre jamais réparée. Il avait fait une étude particulière de ce peuple; longtemps il prit sa défense avec autant de zèle que d'ardeur, non moins pour venger sa gloire que pour améliorer sa condition morale.

Après cette courte introduction, l'auteur livre son ouvrage au lecteur. Cependant la justice ou du moins la franchise exige de lui qu'il recommande à toutes les jeunes personnes dont les idées sont ordinairement resserrées entre les quatre murs d'un salon, à tous les célibataires d'un certain âge qui sont sujets à l'influence du temps, enfin a tous les membres du clergé, si ces volumes leur tombent par hasard entre les mains, de ne pas en entreprendre la lecture. Il donne cet avis aux jeunes personnes qu'il vient de désigner, parce qu'après avoir lu l'ouvrage elles le déclareraient inconvenant aux célibataires, parce qu'il pourrait troubler leur sommeil; aux membres du clergé, parce qu'ils peuvent mieux employer leur temps. (Defauconprêt i-xii). 
Antes de pasar a analizar el prólogo de la traducción española, digamos que Defauconprêt respeta, casi escrupulosamente, el texto original: los diez párrafos de Cooper son convertidos en nueve por el francés, que utiliza un vocabulario prácticamente equivalente al de su modelo, como podemos ver en el siguiente ejemplo del párrafo $4^{\circ}$ :

The tribe that possessed the country which now composes the south-western parts of New-England, and the portion of New-York that lies east of the Hudson, and the country even much farther to the south, was a mighty people called the "Mahicanni," or, more commonly, the "Mohicans." The latter word has since been corrupted by the English, into "Mohegan."

\section{La traducción española del francés de D. Vicente Pagasartundua}

Caso típico de traducción indirecta, que es bien definida por una estudiosa de las traducciones indirectas del chino:

Las traducciones indirectas, también llamadas de segunda mano o relevo, han favorecido la recepción literaria en la extensa historia de las relaciones entre culturas, culturas a veces geográficamente alejadas y con lenguas dispares, o culturas que han sido mediadas por otras de mayor poder o prestigio. Las causas de la existencia de este modo de traducción varían según el contexto; lo curioso, no obstante, es el escaso interés que han despertado entre los investigadores (Marín Lacarta 2008 1).

Por lo que concierne a la difusión y traducción de las obras de Cooper en España, el panorama que describe Montesinos en el capítulo IV ("Del antiguo régimen a las emigraciones") de su estudio sobre la novela española en el XIX es revelador:

Fuera de Walter Scott apenas hay novelista entre los traducidos y publicados entonces en España misma, que merezca recordarse, si no es el americano J. Fenimore Cooper, recién descubierto en Francia -Defauconprêt, infatigable traductor de novelas históricas, trasladó las suyas por los años de 1822 y siguientes-, arribado a España diez años más tarde y copiosamente divulgado por Jordán (es decir, Delgado), que publica en 1832 Los nacimientos del Susquehanna o los primeros plantadores (The Pionneers, 1823), El piloto, El último de 
los mohicanos — del que hay una edición de Valencia, hecha por Orga el mismo año- y La Pradera. Novelista histórico a su modo, Cooper inicia, sobre todo entre nosotros, el gusto por la novela de aventuras en países exóticos — sus libros harán las delicias de la juventud hasta nuestros días- [...] Hombres de letras eminentes acogieron a Cooper con entusiasmo; el mismo Larra hizo, según parece, una traducción de El piloto. (Montesinos 77-78).

La versión de don Vicente Pagasartundua El último de los Mohicanos. Historia de mil setecientos cincuenta y siete, por Fenimore Cooper, traducida del francés, Madrid, Tomás Jordán, 1832 está compuesta por dos tomos, conteniendo el primero XVII capítulos y el segundo XVI de texto francés, que incluye también el prefacio que añade Defauconprêt a su traducción, denominado de igual modo, en lugar del más usual en español "prólogo".

Ya desde el prefacio Pagasartundua sigue fielmente a Defauconprêt, ${ }^{12}$ aunque a veces añada algo de su propia cosecha. Un análisis de la versión española del prólogo francés sacará a la luz los procedimientos utilizados por el traductor español, así como el tipo de versión al que podría adscribirse.

\section{PREFACIO}

No extrañéis el color de mi tez:

es la marca un poco oscura del brillante sol, bajo el cual he nacido. (SHAKESPEARE: El mercader de Venecia)

El lector que emprenda la lectura de estos volúmenes con la esperanza de encontrar en ellos un cuadro imaginario y romancesco de lo que jamás ha existido, la suspenderá sin duda cuando se halle defraudado de la diversión que se había prometido. La obra no es otra cosa que lo que anuncia su título, un relato, una relación. Sin embargo, como contiene detalles que pudieran no estar al alcance de todos los lectores, y especialmente lectoras, pasando en su concepto por una ficción, es de nuestro deber ilustrar lo que las alusiones históricas podrían presentar de oscuro. Este deber es tanto más riguroso cuanto

\footnotetext{
${ }^{12}$ En la transcripción de los prólogos, francés y español, hemos actualizado la acentuación y la ortografía.
} 
que varias veces hemos adquirido la triste experiencia de que, aun cuando el público ignorase completamente los hechos que se le refieren, desde el momento en que se someten a su respetable tribunal, se halla que por una especie de intención inexplicable sabe más que el autor mismo. Este hecho es incontestable; pero sin embargo, si un escritor se arriesga a dar a la imaginación de los otros el vuelo que no debía haber dado más que a la suya, por una nueva contradicción tendrá casi siempre que arrepentirse. Todo lo que puede ser explicado, debe serlo con esmero, bajo la pena de descontentar a aquella clase de lectores que encuentran más placer en recorrer una obra, cuánto esta les ofrece más enigmas que adivinar, o más misterios que penetrar. El autor empezará la tarea que se ha impuesto por la exposición preliminar de las razones que le obligan a emplear desde el principio tantas palabras ininteligibles. Nada dirá que no sepa el que se halle algún tanto versado en el conocimiento de las antigüedades indianas.

La mayor dificultad que tiene que vencer cualquiera que intenta saber la historia de los indios salvajes, es la confusión que reina en los nombres. Si se reflexiona que los holandeses, los ingleses y los franceses, como conquistadores, se han tomado la licencia de mudarlos e invertirlos todos; que los naturales mismos hablan no solamente diferentes lenguas, y aun dialectos de estas mismas lenguas, sino que además de eso les agrada multiplicar las denominaciones, esta confusión causará menos sorpresa que pesadumbre, y podrá servir de escusa para lo que parezca oscuro en esta obra, cualesquiera que sean por otra parte los defectos que pueda contener.

Los europeos hallaron la inmensa región que se contiene entre el Penobscot y el Potomac, el Océano Atlántico y el Misisipi, en la posesión de un pueblo que no tenía más que un solo y mismo origen. Es posible que sobre uno o dos puntos los límites de este vasto territorio hayan sido dilatados o restringidos por las naciones comarcanas; pero a lo menos tales eran los límites naturales y ordinarios. Este pueblo tenía el nombre genérico de Wapanachki, pero afectaba también el de que significaba un pueblo sin mezcla. El autor confiesa francamente que sus conocimientos no llegan hasta el punto de poder enumerar las comunidades o tribus en que esta raza de hombres se ha subdividido. Cada tribu tenía su nombre, sus jefes, su territorio particular para la caza, y aun su dialecto peculiar. Estos pueblos se combatían mutuamente como los príncipes feudales del antiguo mundo, y ejercían los privilegios de la soberanía; 
pero no por eso dejaban de reconocer un origen común; su lengua era la misma, igualmente que sus tradiciones, que se transmitían con una fidelidad extraordinaria. Una rama de este pueblo numeroso ocupaba las riberas de un caudaloso río llamado Lenapewihittuck. Allí era donde por unánime consentimiento estaba establecida la casa larga o el fuego del gran consejo de la nación.

La tribu que poseía el país que forma en nuestros días la parte Suroeste de la nueva Inglaterra, y la porción de Nueva York que está al Oriente de la bahía de Hudson, igualmente que una grande extensión de país que se prolongaba todavía más hacia el Sur, era un pueblo poderoso llamado «Los Mohicanni»o más comúnmente los Mohicanos. De ésta última palabra han formado después los ingleses por corrupción la de Mohegans.

Los Mohicanos estaban también divididos en tribus. Colectivamente estos disputaban la antigüedad aun a sus vecinos que poseían la casa larga; pero unánimemente eran reconocidos como el hijo primogénito de su gran padre. Esta porción de propietarios primitivos del suelo fue la primera desposeída por los blancos. El pequeño número que queda todavía se ha dispersado entre las otras tribus, y de su antigua grandeza y poder no quedan más que tristes recuerdos.

La tribu que guardaba el recinto sagrado de la casa del consejo fue distinguida por mucho tiempo con el título lisonjero de Lenape; pero cuando los ingleses mudaron el nombre del río en el de Delaware, este nombre fue el que insensiblemente adquirieron los habitantes. En general manifiestan mucha delicadeza y descernimiento en el empleo de las denominaciones. Expresivas distinciones dan más claridad a sus ideas, y comunican muchas veces grande energía a sus discursos.

En un espacio de muchas centenas de millas, a lo largo de las fronteras septentrionales de la tribu de los Lenapes, habitaba otro pueblo que presentaba las mismas subdivisiones, el mismo origen, la misma lengua, y que sus vecinos llamaban Mengwe. Estos salvajes del Norte eran sin duda ninguna menos poderosos, y estaban más desunidos entre sí que los Lenapes. A fin de remediar esta desventaja, cinco de sus tribus, las más numerosas y las más guerreras que se hallaban más inmediatas a la casa del consejo de sus enemigos, se ligaron entre sí para defenderse mutuamente; y estas son en efecto las más antiguas repúblicas unidas de que la historia de la América 
septentrional nos da algunas nociones. Estas tribus eran los mohawks, los oneidas, los senecas, los cayugas y los onondagas. Posteriormente, una tribu vagabunda de la misma raza que se había adelantado hacia el Oriente, vino a reunirse a ellos, y fue admitida a participar de todos sus privilegios políticos. Esta tribu, llamada los tuscaroras, aumentó de tal modo su número, que los ingleses cambiaron el nombre que habían dado a la confederación, y no los llamaron ya los cinco, sino las seis naciones. En el progreso de esta relación se verá que la palabra nación se aplica tan pronto a una tribu como a un pueblo entero en su significado más extenso. Los mengwes eran llamados también por sus vecinos macuas, y aun muchas veces por burla mingos. Los franceses les dieron el nombre de iroqueses, por corrupción sin duda de alguna de las denominaciones que tomaban.

Una tradición auténtica ha conservado el detalle poco honroso de los medios que los holandeses por una parte, y los mengwes por otra emplearon para determinar a los lenapes a que depusiesen las armas, encomendando a los últimos el cuidado de su defensa; en una palabra, a no ser ya más que mujeres, en el lenguaje figurado de los naturales. Si la política seguida por los holandeses era poco generosa, era por lo menos sin peligro. Desde este momento trae su fecha la caída de la mayor y más civilizada de las naciones indianas que ocupaban el territorio actual de los Estados Unidos. Despojados por los blancos, oprimidos y asesinados por los salvajes, estos desgraciados continuaron todavía por algún tiempo vagando alrededor de su casa del consejo; y después, separándose en cuadrillas, fueron a refugiarse en las vastas soledades que se dilatan al Occidente. Como el resplandor de una lámpara que se apaga, su gloria no brilló nunca tanto como en el momento en que iba a extinguirse para siempre.

Aún pudieran darse otros detalles sobre este interesante pueblo, en particular sobre la parte más reciente de su historia; pero el autor no los cree necesarios para desenvolver el plan de esta obra. La muerte del piadoso y venerable Heckewelder ${ }^{13}$ es una pérdida bajo este aspecto, que no podrá ser nunca reparada. Este respetable eclesiástico había hecho un estudio particular de este pueblo, y le defendió largo tiempo

\footnotetext{
${ }^{13}$ El reverendo Heckewelder podría ser considerado el Las Casas de la América del Norte, por sus escritos sobre los indios.
} 
con tanto celo como ardor, no menos para vengar su gloria que para mejorar su condición moral.

Después de esta corta introducción, el autor presenta su libro al lector. Sin embargo, la justicia, o a lo menos la franqueza, exige de él que a todas las jovencitas, cuyas ideas están ordinariamente circunscriptas entre las cuatro paredes de un salón; a todos los celibatarios de una cierta edad que están sujetos a la influencia del tiempo; en fin, a todos los miembros del clero, en cuyas manos pueden caer estos volúmenes, les recomiende no emprendan su lectura. Da este aviso a las jóvenes que acaba de designar, porque después de haber leído la obra les parecería mal; a los celibatarios, porque podría turbar su sueño, y a los miembros del clero, porque pueden emplear mejor su tiempo. (Op. cit. 5-14).

El análisis que llevaremos a cabo ${ }^{14}$ se basa en una lectura contrastiva de los prólogos de ambas versiones, que pondrá de manifiesto todas aquellas discordancias localizadas de tipo morfosintáctico y léxicosemántico; sin ceñirnos a un modelo de análisis preestablecido, ni seguir un orden lineal en la búsqueda de discordancias, trascribiremos ejemplos en ambas lenguas que pondrán de relieve las particularidades más representativas del modo de traducir de don Vicente Pagasartundua. ${ }^{15}$

La primera observación que procede es que Pagasartundua encabeza su prefacio con un exergo, que Defauconprêt (y el propio Cooper) colocan en la portada del libro; se trata de dos versos pertenecientes a El Mercader de Venecia de Shakespeare, acto 2, escena $1^{\mathrm{a}}$, correspondientes al inicio del parlamento del rey de Marruecos:

Mislike me not for my complexion,

The shadowed livery of the burnished sun.

${ }^{14}$ Desde el punto de vista de un lector de nuestro tiempo, igual que hemos procedido al actualizar la acentuación y la ortografía.

${ }^{15}$ Según podemos leer en el Diario de Madrid (domingo, 20 de febrero de 1825) era Oficial de la dirección general de Reales loterías. Siguiendo la estela de su modelo francés de traductor, es decir, traduciendo de las versiones de Defauconprêt, versionaría indirectamente del francés obras de Cooper (El piloto, historia marina, por Fenimore Cooper, traducido del francés por D. Vicente Pagasartundua -vol.1- y Manuel Bazo -vol2, Madrid, 1832; imprenta y librería de D. Tomás Jordán, 2 tomos; El último de los mohicanos, historia de 1757 por Fenimore Cooper; traducido del francés por D. Vicente Pagasartundua. Madrid, 1832; imprenta y librería de Tomás Jordán, 2 tomos) y de Walter Scott (Kenilworth..., trad. del francés por don Vicente Pagasartundua. Madrid: Imprenta de Jordán, 1832). 
El traductor francés lo coloca en el mismo lugar que Cooper, siendo su traducción la siguiente:

La couleur de mon teint par vous est censurée

Et pourquoi? Du soleil il porte la livrée.

La versión española, en prosa, es posiblemente bastante más exacta que la francesa. ${ }^{16}$

Pero, tras esta pequeña libertad del traductor español, su fidelidad es tal que respeta escrupulosamente los párrafos de su modelo francés: 10 en cada caso.

Analizados los prólogos en tanto que lectores contemporáneos, se revela la utilización por parte de Pagasartundua de un procedimiento traductológico heterogéneo, pues la técnica de trasvase empleada, al no evidenciar un patrón traslativo recurrente, resulta un tanto arbitraria; sin embargo, a grandes rasgos, es posible afirmar que la versión traducida concede primacía a la equivalencia semántica y a la simetría sintáctica, particularidad, esta última, que desemboca en una literalidad que priva en ocasiones al texto meta de naturalidad lingüística.

Para dar muestra de dicha arbitrariedad, nos servimos del componente léxico de la traducción; la elección terminológica de Pagasartundua pone de manifiesto, por un lado, una intención de respeto absoluto por la conservación del léxico original, y, por otro, el propósito de mejorar la exactitud y precisión de los términos esgrimidos por el autor del texto francés, para cuya traslación, con frecuencia, la traducción literal habría sido una opción totalmente aceptable. A modo de ejemplo de tales particularidades contradictorias, trascribimos el siguiente ejemplo:

Le lecteur qui commence la lecture de ces volumes dans l'espoir d'y trouver le tableau romanesque et imaginaire de choses qui n'ont jamais existé l'abandonnera sans doute lorsqu'il se verra trompé...

${ }^{16}$ No habría sido muy difícil trasladar la cita en verso, obteniendo sonoras aliteraciones en $t$ :

"No os mostréis asombrado del color de mi tez, / porta el tiznado atuendo de mi bruñido sol". 
El lector que emprenda la lectura de estos volúmenes con la esperanza de encontrar en ellos un cuadro imaginario y romanesco de lo que jamás ha existido, la suspenderá sin duda cuando se halle defraudado...

Posiblemente, la elección del léxico subrayado, que no resulta por su significado desacertada, evidencia el propósito del traductor de perfeccionar la utilización terminológica del texto meta, pues es ciertamente inusual que no opte por la traducción literal ('commencer' por 'comenzar' y 'abandonner' por 'abandonar') cuando esta se erige como una posibilidad tan espontánea como correcta. Al contrario ocurre en los ejemplos que se muestran a continuación, en los que el traductor, en un intento de reproducir fielmente el léxico original, incurre en evidentes errores semánticos:

Une tradition authentique a conservé le détail des moyens peu honorables que les Hollandais d'un côté, et le Mengwes de l'autre, employèrent pour déterminer les Lenapes à déposer les armes...

Una tradición auténtica ha conservado el detalle poco honroso de los medios que los holandeses por una parte, y los mengwes por otra emplearon para determinar ${ }^{17}$ a los lenapes a que depusiesen las armas...

En otras ocasiones, la literalidad del traductor no desemboca en incorrecciones semánticas, sin embargo, merma significativamente la calidad de la versión española, que puede resultar un tanto artificial a los ojos del lector meta:

Cette portion des propriétaires primitifs du sol fut la première dépossédée par les blancs.

Esta porción de propietarios primitivos del suelo fue la primera desposeída por los blancos.

Dépouillés par les blancs, opprimés et massacrés par les sauvages, ces malheureux continuèrent encore quelque temps à errer autour de leur maison du conseil...

${ }^{17}$ La 5. ${ }^{a}$ edición del Diccionario de la Lengua Castellana de la Real Academia Española (1817), elegida ésta por ser la más próxima a la publicación de la traducción, recoge únicamente las siguientes acepciones para el verbo 'determinar':

-Resolver lo que se ha de hacer en alguna cosa.

-Distinguir, discernir alguna cosa.

-Señalar, fijar alguna cosa para algún efecto.

-Resolver la indiferencia de alguna cosa o contraerla a determinada especie.

-Sentenciar, definir. 
Despojados por los blancos, oprimidos y asesinados por los salvajes, estos desgraciados continuaron todavía por algún tiempo vagando alrededor de su casa del consejo...

Des nuances expressives donnent plus de clarté à leurs idées, et communiquent souvent une grande énergie à leurs discours.

Expresivas distinciones dan más claridad a sus ideas, y comunican muchas veces grande energía a sus discursos.

En el primer ejemplo transcrito, el término 'dépossédée', traducido por Pagasartundua literalmente, 'desposeída', habría sido vertido al español de forma más acertada de haber utilizado un término tal como 'expropiada' 18 o 'confiscada'; ${ }^{19}$ un adjetivo de significado análogo a los anteriores habría constituido una opción más adecuada para el vocablo francés que inaugura el segundo ejemplo, 'dépouillés', en lugar de 'despojados', ${ }^{20}$ que parece truncado al no aparecer junto a un complemento directo que concrete su significado. Por último, las opciones 'dotar', 'conferir' u 'otorgar' resultan, por el contexto de uso, significativamente más pertinentes que 'comunicar'.

Respecto del trasvase sintáctico, se observa igualmente un procedimiento aleatorio por parte del traductor que, en algunas ocasiones, decide distanciarse del texto original al optar por estructuras de evidente naturalidad lingüística en el idioma de llegada, y, en otras, se doblega hasta tal punto ante la sintaxis del texto francés que llega incluso a incurrir en errores sintácticos de gran importancia.

Se puede señalar como un gran acierto del traductor la exacta traslación de los galicismos de construcción o locuciones enfáticas del tipo "c'est...que". Este procedimiento enfático que abunda en la lengua francesa tanto a nivel escrito como oral, constituye una de las dificultades más recurrentes con las que debe enfrentarse el traductor

${ }^{18}$ Lógicamente, Pagasartundua no pudo emplear este verbo pues, al no quedar recogido en la 5 . $^{a}$ versión del Diccionario citada, suponemos que no existía.

${ }^{19}$ El termino más acertado en este caso sería, sin duda, 'confiscar', pues dicho Diccionario alude a la "privación de bienes", que es específicamente a lo que se hace referencia en el contexto en cuestión, entendida la 'tierra' como un bien material.

${ }^{20}$ La citada edición contempla la acepción siguiente para el verbo 'despojar', “Quitar y privar a alguno de lo que goza y tiene, desposeerle con violencia", sin hacer referencia alguna a aquello de lo que se quita o priva al sujeto, por lo que consideramos que la omisión del complemento objeto en este caso, hace de este verbo desacertado para la comprensión del sentido original (la privación de tierras). 
de lengua francesa, que generalmente opta inadecuadamente por conservar el orden sintáctico, que tan poco natural lingüísticamente resulta al lector español. Pagasartundua, sin embargo, determina trasladar dichas estructuras por medio de la adaptación de la oración francesa al uso cotidiano del idioma de llegada, como ilustra el ejemplo que trascribimos a continuación, en el que se observa cómo se invierte el orden de la frase española para eludir tal obstáculo sintáctico. Así pues, frases de este tipo que la mayor parte de los traductores suelen verter mal a nuestra lengua, son traducidas por Pagasartundua con total corrección, como vemos por los siguientes ejemplos:

C'est par l'exposé préliminaire des raisons qui l'obligent dès le début à employer tant de mots intelligibles que l'auteur commencera la tâche qu'il s'est imposée.

El autor empezará la tarea que se ha impuesto por la exposición preliminar de las razones que le obligan a emplear desde el principio tantas palabras ininteligibles.

C'était la que d'un consentement unanime était établie...

Allí era donde por unánime consentimiento estaba establecida...

C'est de ce dernier mot que les Anglais ont fait...

De esta última palabra han formado después los ingleses...

C'est de ce moment que date la chute de la plus grande...

Desde este momento trae su fecha la caída de la mayor...

Asimismo en otro galicismo de construcción que actualmente utiliza, tanto en la escritura como en la conversación cualquier hablante de habla española, el traductor evita el repetido e incorrecto uso de la estructura de un lado, en lugar de por una parte:

Une tradition authentique a conservé le détail des moyens peu honorables que les Hollandais d'un côté, et les Mengwes de l'autre...

Una tradición auténtica ha conservado el detalle poco honroso de los medios que los holandeses por una parte, y los mengwes por otra...

Ante tal destreza traslativa, es desconcertante el hecho de que Pagasartundua incurra con cierta frecuencia en errores sintácticos que atañen a elementos tan baladíes como el uso preposicional en la lengua meta; los siguientes ejemplos ponen de manifiesto un uso erróneo de la preposición en español, motivado probablemente por la preposición regida en la lengua original: 
Par la suite une tribu vagabonde de la même race, qui s'était avancée plus près du soleil, vint se joindre à eux, et fut admise à participer à tous leurs privilèges politiques.

Posteriormente, una tribu vagabunda de la misma raza que se había adelantado hacia el Oriente, vino a reunirse a ellos, y fue admitida a participar de todos sus privilegios políticos. [En lugar de "reunirse con ellos"].

Les Européens trouvèrent cette région immense qui s'étend entre le Penobscot et le Potomac, l'Océan Atlantique et le Mississipi, en la possession d'un peuple qui n'avait qu'une seule et même origine.

Los europeos hallaron al inmensa región que se contiene entre el Penobscot y el Potomac, el Océano Atlántico y el Misisipi, en la posesión de un pueblo que no tenía más que un solo y mismo origen. [En lugar de "bajo la posesión"].

De manera general, y como característica más significativa, destacamos una literalidad excesiva que redunda, en algunas ocasiones, en la carente naturalidad lingüística del texto español. El traductor concede primacía al léxico y a la sintaxis de la obra francesa, por lo que no es de extrañar que detectemos vocablos y estructuras que oscurecen y dificultan en parte la comprensión de la versión española, tales como los que trascribimos a continuación:

Tout ce qui peut être expliqué doit donc l'être avec soin, au risque de mécontenter cette classe de lecteurs qui...

Todo lo que puede ser explicado, debe serlo con esmero, bajo la pena de descontentar a aquella clase de lectores que...

Une tradition authentique a conservé le détail des moyens peu honorables que les Hollandais d'un côté, et les Mengwes de l'autre, employèrent pour déterminer les Lenapes à déposer les armes...

Una tradición auténtica ha conservado el detalle poco honroso de los medios que los holandeses, por una parte, y los mengwes, por otra, emplearon para determinar a los lenapes a que depusiesen las armas...

Las incorrecciones ocasionadas por el apego excesivo a la lengua del texto original por parte del traductor, son aún más perceptibles en el nivel morfosintáctico. Los siguientes ejemplos evidencian que Pagasartundua, en un intento de reproducir fielmente las estructuras sintácticas de la obra francesa, infringe la normativa española. 
Afin de remédier cette désavantage, cinq de leurs tribus les plus nombreuses et les plus guerrières qui se trouvaient...

A fin de remediar esta desventaja, cinco de sus tribus, las más numerosas y las más guerreras que se hallaban...

En este caso, el traductor se sirve de una aposición explicativa ("las más numerosas y las más guerreras") para trasvasar al español la estructura francesa de superlativo de superioridad "les plus + adj".

On verra dans le cours de cette relation que le mot nation s'applique tantôt à une tribu et tantôt au peuple entier...

En el progreso de esta relación se verá que la palabra nación se aplica tan pronto a una tribu como a un pueblo entero...

Pagasartundua traduce de forma aislada el adverbio 'tantôt' (tan pronto) sin tener en consideración que forma parte, junto al segundo adverbio 'tantôt' que figura más adelante, de una correlación de adverbios que funcionan como conjunciones coordinadas. Habría sido más apropiado traducir dicha correlación como "tanto a... como a...".

Motivada, igualmente, por dicha literalidad, se observa cierta tendencia por parte del traductor a la utilización incorrecta de los complementos de régimen verbal; en el ejemplo que transcribimos a continuación, se aprecia cómo Pagasartundua traslada, sin modificación alguna, la preposición regida por el verbo francés, incluso cuando ésta no es la regida por el verbo español por el que ha optado.

Par la suite une tribu vagabonde de la même race, qui s'était avancée plus près du soleil, vint se joindre à eux...

Posteriormente, una tribu vagabunda de la misma raza que se había adelantado hacia el Oriente, vino a reunirse a ellos...

Por otro lado, es preciso resaltar que Pagasartundua no respeta generalmente la grafía cursiva, la mayúscula y las comillas del texto francés, lo que no impide la comprensión de la versión española, mas sí atenta con la intención original de Defauconprêt.

L'ouvrage n'est autre chose que ce qu'annonce son titre, une relation.

La obra no es otra cosa que lo que anuncia su título, un relato, una relación.

C'était là que d'un consentement unanime était établie «la Maison Longue» ou «le Feu du Grand Conseil» de la nation. 
Allí era donde por unánime consentimiento estaba establecida $\underline{\text { la }}$ casa larga o el fuego del gran consejo de la nación.

En el primer ejemplo se aprecia que el traductor opta por omitir la cursiva, mientras que, en el segundo, la utiliza deliberadamente para trasvasar el texto entre comillas; el segundo ejemplo muestra, igualmente, su preferencia por suprimir la mayúscula.

En confrontación con lo anteriormente dicho, es de especial enjundia añadir en último término que, pese a la abusiva literalidad que ahoga la naturalidad lingüística de la traducción, no son frecuentes las omisiones ni las adiciones semánticas en el texto español; detectamos ocasionalmente alteraciones de significado, aunque el contexto suele paliar tales desaciertos, como se observa en los siguientes ejemplos:

Les Européens trouvèrent cette région immense qui s'étend entre le Penobscot et le Potomac...

Los europeos hallaron la inmensa región que se contiene entre el Penobscot y el Potomac...

[...] cette classe de lecteurs qui trouvent d'autant plus de plaisir à parcourir un ouvrage qu'il leur offre plus d'énigmes à deviner ou plus de mystères à éclaircir.

[...] aquella clase de lectores que encuentran más placer en recorrer una obra, cuando esta les ofrece más enigmas que adivinar, o más misterios que penetrar.

Añadamos, para finalizar este breve análisis, el uso correcto de los tiempos verbales y el vocabulario, salvo el leísmo del penúltimo párrafo: "le defendió".

\section{Conclusiones}

Si casi todas las novelas inglesas que se tradujeron en España a lo largo del siglo XVIII se realizaron a través de la versión francesa (en la mayor parte de los casos con escasa fidelidad al texto original), nada de extraño tiene que durante las primeras décadas del XIX se siguiera la misma pauta, ampliándose en el caso concreto de la traducción de los primeros autores estadounidenses.

En el caso que nos ocupa, la traducción de un texto inglés a través de un "intermediario" francés, como es del todo lógico la exactitud de la 
versión española depende de la de su modelo francés; a este respecto, se puede afirmar que Pagasartundua se basó en un gran traductor, reputado y con un gran dominio de la lengua inglesa, lo que seguramente lo llevaría a olvidarse por completo del texto original.

El análisis contrastivo del prólogo, aun sin ser un análisis contrastivo completo del prólogo, nos puede servir para extraer conclusiones del modo de traducir de Pagasartundua. Si aceptamos como modelo de tipología en la traducción el que propone Inmaculada Urzainqui en un trabajo dedicado a la traducción en el siglo XVIII, la versión de nuestro traductor podría incluirse en el tipo de traducción que Urzainqui denominaría “Traducción-traducción”, y que responde a una voluntad de fidelidad y respeto al original, por más que pueda dar cabida a diversas libertades introducidas por imperativos estéticos o de congruencia lingüística, y que ella define como: "traducción fiel, exacta y puntual, respetuosa al máximo con el original” (1991 637).

\section{OBRAS CITADAS}

Cointre, Annie, et Rivara, Annie. Recueil de Préfaces de traducteurs de romans anglais. Saint-Étienne: Publications de l'Université de Saint-Étienne, 2006.

Defauconprêt, Auguste-Jean-Baptiste. Le Dernier des Mohicans, histoire de 1757. Paris: Charles Gosselin, 1826.

García Yebra, Valentín. En torno a la Traducción. Teoría. Crítica. Historia. Madrid: Gredos, 1989.

Klibbe, Lawrence H. "La visión de España en la novela de Cooper, Mercedes of Castile". Actas del X Congreso de la Asociación internacional de Hispanistas, Barcelona 21-28 de agosto de 1989, coord. por Antonio Vilanova, vol. 2, 1992.

Kittel, Harald. "Indirect Translation in Eighteenth-Century Germany". Interculturality and the Historical Study of Literary Translations. Berlin: Eric Schmidt, 1991, 3-25.

Lefebure, Maurice-Jean. Structure du discours de la poésie et du récit. Neuchâtel: La Baconnière, 1971. 
Marín Lacarta, Maialen. "La traducción indirecta de la narrativa china contemporánea al castellano: ¿síndrome o enfermedad?”. Revista de Historia de la Traducción, 2, 2008.

Fernández Montesinos, José. Introducción a una historia de la novela en España en el siglo XIX. Seguida del esbozo de una bibliografía española de traducciones de novelas 1800-1850. Madrid: Editorial Castalia, 1982.

Pagasartundua, Vicente. El último de los Mohicanos. Historia de mil setecientos cincuenta y siete, por Fenimore Cooper, traducida del francés. Madrid: Tomás Jordán, 1832.

Taylor, Edward G. The Works of the Rev. Sydney Smith. New York: D. Appleton and company, 1846.

Tesnière, Lucien. Éléments de syntaxe structurale. Paris: Klincksieck, 1959.

Urzainqui, Inmaculada. "Hacia una tipología de la traducción en el siglo XVIII: los horizontes del traductor". Traducción y adaptación cultural: EspañaFrancia. Oviedo: Universidad de Oviedo, 1991, 623-638.

Viñuela Angulo, Urbano. "James Fenimore Cooper: Entre la popularidad y la transformación textual”. Livius, 4, 1993.

Received: 29 July 2014 Accepted: 03 November 2014 


\title{
HOUSES WITH PICKET FENCES: TRYING TO RECOVER A PAST THAT NEVER WAS IN PLEASANTVILLE AND CELEBRATION, FLORIDA
}

\author{
M. Carmen Gómez Galisteo \\ $U N E D$
}

\begin{abstract}
When one thinks about traditional values and perfect families in the United States of America, the image that immediately comes to mind is that of the Fifties, a decade repeatedly portrayed in an endless number of Hollywood movies as the epitome of an idyllic past. Because the filmic representation of this period is more often than not sugarcoated and far too perfect, the movie Pleasantville (1998) denounces the pervasiveness of this fabricated image of the Fifties. Yet, the so-called utopian neighborhoods of the Fifties continue being an architectural goal, as the design and founding of the planned community of Celebration, Florida, testify to. This essay uses Pleasantville and the example of Celebration to explore how the Fifties are still very much present in contemporary American culture and life.
\end{abstract}

Key words: Pleasantville, the fifties, Hollywood, new urbanism, town planning, Celebration.

\section{Resumen}

$\mathrm{Al}$ pensar en valores tradicionales y familias perfectas en Estados Unidos, la imagen que de inmediato viene a la mente es la de los años cincuenta, una década repetidamente reflejada en un gran número de películas de Hollywood como la encarnación de un pasado idílico. Sin embargo, dado que la representación fílmica de este periodo es a menudo edulcorada y demasiado perfecta, la película Pleasantville (1998) denuncia esta imagen artificial de los cincuenta. A pesar de 
ello, las presuntamente utópicas comunidades de los cincuenta siguen siendo un objetivo en el campo de la arquitectura, tal y como el ejemplo de la ciudad de Celebration en Florida sugiere. Este artículo analiza Pleasantville y Celebration para explorar cómo la década de los cincuenta aún está muy presente en la cultura y la vida americana en la actualidad.

Palabras clave: Pleasantville, años cincuenta, Hollywood, urbanismo y planificación urbana, Celebration.

Once upon a time in the Fifties there were beautiful suburban houses where nuclear families lived happily ever after... Or so has Hollywood told us over and over again. As a result of having watched so many movies set in the Fifties, it has turned out that nowadays probably the most salient and easily recognizable aspect of life then is friendly neighborhoods of row after row of identical houses only told apart by the color of the façade, inhabited by ideal families at the core of which are perfect housewives. But up to which extent is this a fabrication we have more or less willingly partaken of? Have we become so infatuated with these recreations of the Fifties that we take them for reality? Pleasantville (Ross, 1998) tackles the depiction of this decade to denounce that small-town life in the Fifties was not as idyllic as we have been led to believe.

Pleasantville addresses the problem of how we remember the Fifties through the character of David, a 1990s teenager who is a big fan of a (fictional) TV show set in the Fifties, "Pleasantville", about daily life in the homonymous town. The problem is that, after having watched so many recreations of the Fifties, many viewers find it hard to bear in mind that the Fifties were not exactly as TV or movies present them and this is what happens to David. While aware that "Pleasantville" is a show, David still gets comfort from watching it. ${ }^{1}$ Nonetheless, it is not clear if he knows that the show does not accurately capture life in the Fifties, but is an idyllic recreation. Because "Pleasantville" is a TV show

${ }^{1}$ By "Pleasantville", we refer to the fictional show David watched; by Pleasantville, to the movie; and by Pleasantville, without italics and without inverted commas, to the fictional town. 
and not real life, to bring attention to the artificiality of David's image of the Fifties as derived from "Pleasantville", "Pleasantville" is a blackand-white TV show. Therefore, once David and his twin sister, Jennifer, thanks to a magic remote control, get into the show and become the perfect Bud and Mary Sue, the children of the not less perfect Mr. and Mrs. Parker, they discover that Pleasantville is in black and white. In sharp contrast, in the movie "colour is used as a form of spectacular excess in a black and white past that is itself fantastical" (Grainge 2003: 205).

David is unable to remove himself from the show's appeal because he badly wants to believe that the Fifties offered everything he lacks, starting with a loving family instead of his divorced parents. So, once he becomes a character in the show, he struggles to keep Pleasantville as close to "Pleasantville" as possible, fighting the changes and further contributing to perpetuate this misleading image (although he inadvertently brings about the first change when he urges Skip not to date his sister). Pleasantville ultimately changes because newcomers bring with them novel ideas and set in motion a chain of changes. The utopia of Pleasantville soon becomes a dystopia, as "certain things have become... 'Un-Pleasant," in the words of the Pleasantville mayor, Big Bob.

Suburbs were a dominant feature of life in America in the Fifties, replacing other housing types up to the point that "by 1950, 40 to 50 million Americans lived in the suburbs" (Quart and Auster 1991: 45). ${ }^{2}$ Although living in the suburbs might at first seem trivial, with no deeper implications and indistinctive from other housing types, it plays a vital role, for "building and living in suburbia depend on the creation and enactment of a suburban imagination, of what Amy Kenyon calls suburban dreams" (Dickinson 2006). Because suburbs are a central feature of American life, a number of movies have been set there, examining their characteristics and specific idiosyncrasies, up to the extent that "at the end of the twentieth century, Douglas Muzzio and Thomas Halper argue, suburbs became a 'cinematic fixation"'(Dickinson 2006). ${ }^{3}$ Consequently, it is not surprising that they occupy much filmic

\footnotetext{
${ }^{2}$ Current figures indicate that "today, more Americans live in suburbs than in urban centers or rural countryside combined" (Dickinson 2006).

${ }^{3}$ Television has also found in the underlying conflicts in the suburbs a goldmine, as epitomized by the success of TV shows such as Desperate Housewives (2004-2012) or Devious Maids (2013 - ).
} 
space in movies such as Far From Heaven(Haynes, 2002), The Truman Show(Weir, 1998), Edward Scissorhands(Burton, 1990), American Beauty (Mendes, 1999) or Revolutionary Road(Mendes, 2008), with Pleasantville being "prototypical of the films that take suburbia as their theme" (Dickinson 2006).

Nostalgic attempts to return to the Fifties are not as far-fetched as we might think. The Disney-founded city of Celebration, Florida, bears testimony to this nostalgic drive that urban planners also aspire to. If Pleasantville itself was defined as a 'suburban Disneyland' (Taylor n.d.), Celebration is actually the realization of Disney suburbia. The city soon attracted an intense media interest as well as a growing bibliography, including some books authored by local residents, such as professor Andrew Ross' The Celebration Chronicles: Life, Liberty, and the Pursuit of Property Value in Disney's New Town and journalist Douglas Frantz's Celebration, U.S.A.: Living in Disney's Brave New Town (co-written with his wife, Catherine Collins).

Creating a perfect community had been Walt Disney's ambition for years, almost his hobbyhorse. Instead of trying to transform an existing place into an ideal community, he intended to create a perfect, brand-new town from scratch. His first project received the name of EPCOT, short for Experimental Prototype Community of Tomorrow (alternatively called Experimental Prototype City of Tomorrow). Originally, Walt envisaged it as a place that

will be a community of tomorrow that will never be completed, but will always be introducing and testing and demonstrating new materials and systems. ... It will be a planned, controlled community, a showcase for American industry and research, schools, cultural and educational opportunities. In EPCOT, there will be no slum areas because we won't let them develop. (quoted in "Epcot")

His plans, however, were put to a halt because of a double-fold problem - lack of funding and the Florida government's demand that Magic Kingdom be built prior to the development of EPCOT. Further undermining the project, Walt did not get to see Magic Kingdom open its doors and, after his death, the company was reluctant to get involved in such an ambitious project as creating an ideal city. When it was finally inaugurated in 1982, EPCOT, had, however, gone a far way from Walt's original idea. His plans for a high-tech city had become reduced to the 
third most visited theme park in the world, called the permanent world fair (in contrast to the official world exhibition, held every four years).

It would not be until the 1990s when Disney started to develop their next attempt at an ideal community, Celebration. Located in Osceola County, Florida, Celebration is a planned community serving as "an example of New Urbanism, or neotraditional town design" (Craven n.d.). ${ }^{4}$ Famous architects were commissioned to develop the first phase, begun in 1996, and, thus, "Michael Graves created the Post Office, Philip Johnson the Town Hall and Robert A.M. Stern Architects designed Celebration Health, a full-service medical center. The Stern firm and Cooper Robertson, both of Manhattan, designed the master plan" (Riddle 1999).Nevertheless, Celebration is not an exact match to Walt's ideas about a new, ideal community. While the spirit and the rationale behind it are the same, Walt's ideal city had a futuristic, state-of-the-art outlook that Celebration, with its traditional flavor and New Urbanist look, is very far from evoking. The urban planners of Celebration were so thorough in creating a picture-perfect city that they seriously considered creating a made-up past for the city. Thus, "one back story would have pretended that survivors of a Spanish shipwreck founded Celebration, and another had it that the town was built on the rubble of Sherman's March" (Andersen 1999). However, they eventually abandoned the idea. There is a certain Disney feeling to the city, too, and Market Street closely resembles Disney World's own Main Street. ${ }^{5}$ Celebration has been the recipient of numerous architectural awards, including the NAIOP Excellence Award for 1996 Development of the Year, the Grand Project Excellence Award in 1997 Large/Design/Civil Surveying for the first phase and the 1998 Award of Excellence granted by the American Society of Landscape Architects, among others ('Tour of Celebration'). Moreover, the Urban Land Institute chose it as the New Community of the Year 2001 ('Celebration, Florida').

Celebration was "designed in a way to encourage a sense of community" (Riddle 1999), a point Disney executives are careful to repeatedly mention. If there were no homeless and no 'coloreds' in

4 "The Truman Show uses New Urbanist architecture and city planning as its visual framework. New Urbanism is a memory-tinged mode of design" (Dickinson 2006).

${ }^{5}$ There is no Main Street in Celebration, though, because Florida regulations do not allow for two streets in the same county bearing the same name and there was already a Main Street in Osceola County by the time Celebration was founded. 
Pleasantville, ${ }^{6}$ the same is true of Celebration:"there are no poor in Celebration, and the town is extremely white-[which] is nevertheless rare today in the suburbs" (Pollan 1997). Announcements of its creation received an immediate, enthusiastic response from 5,000 prospective buyers who were willing to move into one of the 350 homes (apartments and houses) of the initial building phase of Celebration in 1996. A lottery system had to be established to determine who would be given the opportunity to buy them even though a house in Celebration sold for a price considerably higher than a house of similar characteristics in the same county. Other problems eager buyers overlooked include that it "offers few real places to shop - there's no hardware store or pharmacy—and few serious employment opportunities" (Pollan1997).

Even if education at Pleasantville high school is portrayed in an unflattering light (the pages of the books in the library are blank and nothing is known about the world beyond Pleasantville, not even about the United States), education in present-day America is not any better, the movie contends, as in David's twentieth-century classes "students in the high schools are disengaged from their education, a disengagement fostered by teachers who drone apocalyptically about the future" (Dickinson 2006). The trap of romanticizing or idealizing the past is further helped by the 'imperfect' present, and, furthermore, by the utterly negative (when not despairing) predictions the teachers cast.

It is telling of our present-day real concerns and of the imagined concerns of the Fifties that the main point of contention in Pleasantville and the issue which causes the most visible (in a literal sense) changes is sex whereas in Celebration the first dissension had to do with educative policies and the school curricula. If we laughed at the naïve vision of geography put forward in Pleasantville, where Mary Sue gets mocked and laughed at by her classmates for failing to realize that, obviously, the end of Main Street is the beginning of Main Street, this situation is far from being so risible, or so did the Celebration school board seem to believe. Students of different ages studied together in classrooms where textbooks and grades were nonexistent and learning aims and goals

${ }^{6}$ As David tells his friend, "he's not homeless, Howard, they just don't say where he lives. Well, it's a silly question. Because nobody's homeless in Pleasantville." 
were set by each student on an individual basis (Andersen 1999; Pollan 1997; Riddle 1999).

While Pleasantville residents were perfectly happy to remain in their town, happy with their ignorance of anything that happens beyond it, Celebration residents are well aware that one day they will send their children to universities outside their community where other kinds of knowledge will be required. ${ }^{7}$ Complaints against the school and, by extension, against Disney, soon erupted. Although the Celebration K12 school was public, it was a central feature in Disney's marketing strategy, naming it as "a school for tomorrow" and "as a model for education into the next century," both qualities especially remarkable in a state whose schools have often been found lacking (Pollan 1997).Soon the dispute got the media's attention and was counteracted by the population's socalled spontaneous backing (Pollan 1997).While the school curriculum controversy apparently ended with the dissidents' children dropping out of the Celebration school, amounting to 30 students or $16 \%$ of the student body at first (and some families altogether leaving the city) (Pollan 1997), it remains to be seen if there will be further discrepancies that will rock the boat and spoil this utopian community. ${ }^{8}$

Education is certainly an important issue in Pleasantville, although the movie's more explicit portrayal of the sexual awakening of the community (and their subsequent going colored) and its advocacy of tolerance, have somehow overshadowed the equally central role of education. Jennifer, not too interested in books or in anything other than dating in real life, discovers the power of the written word and her passion for literature up to the point that she decides to stay behind in Pleasantville to pursue a university degree. ${ }^{9}$ Pleasantville residents' personal epiphanies happen when they learn about sex, but for Mary Sue, formerly sexually active Jennifer, her epiphany is caused by a discovery of the power of books, which testifies to director and

\footnotetext{
${ }^{7}$ While Truman in The Truman Show is prevented from leaving whenever he tries to, no such obstacles seem to exist in Pleasantville. Pleasantville residents simply do not want to ever leave their town.

${ }^{8}$ The principal and six of the nineteen teachers also resigned (Pollan 1997).

${ }^{9}$ Actress Reese Witherspoon, who played the role of Jennifer, also acknowledged that "when [the kids in the movie] start reading and learning more about themselves and becoming more self-actualized, their insides fill in and start to turn to color and sort of bring their insides out... It's about finding individuality and identity" (quoted in Kahlenberg 1998).
} 
screenwriter Gary Ross' commitment to literacy. Given that he has been regarded as "a crusader for young readers. He's served on the Los Angeles Board of Library Commissioners and donated the proceeds from a special premiere of Pleasantville to help triple the size of the Central Branch's Teen'Scape area for teenagers" (Kahlenberg 1998), it is hardly surprising that his first movie conveyed this message.

While Pleasantville proves to be suffocating enough for David to want to return home, Celebration residents, on the other hand, are, at least in theory, like-minded people who find it attractive to live in a make-believe city promoted by Disney, the quintessential name for harmony, perfection and a childlike universe where nothing bad is supposed to happen. Disney's love for control sat very well with Celebration residents, who, what is more, found it reassuring. In the words of former local resident Roger Burton, "I knew Celebration was going to be a very controlled situation, but controlled in a good way" (quoted in Pollan 1997).It is telling that residents complained that the educative system, as it was a public school, was beyond the control of Disney, looking up to the corporation for a solution to all their problems: 'II moved here because I loved Disney — we had such blind faith,' said Mrs. Shaw, treasurer of the association board" (Goodnough 2004).

Because nobody forces any Celebration resident to live there, to start with, and therefore, to comply with the rules, Celebration has been called "a free-market experiment in enforced niceness, a radical modern reproduction of conservative styles"(Andersen, 1999). Celebration also constitutes "a case study in the ongoing American contradictions between community and freedom" (Andersen 1999), as illustrated by the first problems and the way citizens allowed Disney to have the lion's share when it came to the control over the community. Because Celebration neighbors rather happily relinquished control of the city to Disney, "the measure of democracy is not self-rule but responsiveness - they're prepared to surrender power over their lives to a corporation as long as that corporation remains sensitive to their needs" (Pollan 1997).In its early days, Celebration owners' voting opportunities were quite limited, as Disney had most of the votes until September 2004, when "residents were given a 3-to-2 majority on the homeowners' association board" (Goodnough 2004). For as long as the corporation found it convenient to hold power until they decided to willingly give it back to the homeowners, Disney's rule adopted the form of the benevolent tyrant. 
Yet, Disney is not alone in aspiring to the Fifties' family life, as this return has been advocated by numerous conservative and religious groups. Pleasantville is different from other movies dealing with the Fifties in that it does not really deal with the Fifties, but with the Fifties as they have been imagined and recreated over and over again. Pleasantville is not solely concerned with attacking the Fifties or this sugarcoated vision of the decade; instead, it is about how cultural memories of the Fifties were used in the 90s by conservative groups (Grainge 2003: 210). Accordingly, "the film invokes a variety of issues, images and right-wing bugbears in a fable that responds openly to culture war discourse of the early 1990s" (Grainge 2003: 210). Pleasantville ultimately becomes not just a critique of misconceptions about the Fifties, but also a warning against contemporary political ideologies which "satirises the fallacious nostalgia of the New Right, attached as it was (and remains) to a prelapsarian order of patriarchal norms and family idealism" (Grainge 2003: 212).

While it may be tempting to run after this apparently perfect version of the Fifties, tensions were not far behind, as denoted by the hasty way in which Pleasantville becomes utterly transformed within a few days, anticipating and hinting at the changes that would come to shatter the Fifties' way of life in the two following decades. Because these erroneous reinterpretations of the past might be misleading, Pleasantville does not really question the Fifties but rather the pervasiveness of this idealized portrayal of the Fifties: "Pleasantville creates a hyperreal past, entirely defined by, and within, the fictional conventions of television (sitcom) genre" (Grainge 2003: 208). Pleasantville points the finger at fabricated cultural memories, in common with movies such as "Back to the Future, Thelma and Louise, Who Framed Roger Rabbit?, Forrest Gump ... [which] are all defined by their use and manipulation of the multifarious images and texts that circulate in the contemporary cultural terrain" (Grainge 2003: 207-208). That Ross dealt with Fifties TV shows and not the actual Fifties has much to do with his own, personal background (and his screenwriter father): "for a Hollywood insider like Ross, show business is the only frame of reference. From that perspective, it is nothing less than a patriotic chore to correct the outmoded entertainment of the 50s which has lodged itself in the mind of the fundamentalist right as the simulation of the American past" (Hoberman 1999). 
While Celebration residents did not mind surrendering their rights and their decision power to a tyrant as long as this was a benevolent one, Pleasantville denounces those political views that, in advocating for tradition, actually conceal repression, inequality and other dangers, all in the name of 'tradition'(Porter 2007: 412). This view is best conveyed through the character of the mayor, Big Bob, whose "appeal to a traditionalist form of ethical life is so problematic; he simply takes for granted the inherent goodness and authority of tradition, never reflecting on the fact that his cherished traditional values are pervaded by patriarchal assumptions" (Porter 2007: 412). Because the mantle of tradition may conceal many sins and a less than perfect reality, Pleasantville questions what tradition is and what it entails -and whether it was all $100 \%$ good, to start with.

In analyzing Pleasantville's political agenda or at least its warning against political ideologies, it should also be taken into account that this was Ross' "first directorial outing, after writing speeches for Democratic candidate Michael Dukakis and crafting sound bites for President Bill Clinton" (Hoberman 1999).The movie was seen as resonant of Clintonian America and Clintonian film (a genre inaugurated by Dave [Reitman, 1993], whose screenplay Ross wrote). ${ }^{10}$ Therefore,

as a political statement Pleasantville is seemingly haunted by the spectre of the Reverend Pat Robertson's cable network (a mix of Christian fundamentalism and family-oriented television shows from the black-and-white 50s) and responding to the early stages of the 1996 presidential campaign in which Republican candidate Bob Dole focused his geriatric fire on the "mindless violence and loveless sex" being foisted on the unwilling American people by liberal Hollywood. (Hoberman 1999)

Although Disney no longer holds the tight control over Celebration that its conception auspiced, the town is still considered a Disney-like place: "with its picture-perfect streets, lined with homes fronted by porches, picket fences, and manicured lawns, Celebration is, by design, bathed in nostalgia, almost an extension of Disney World's idealized Main Street U.S.A." (Spindel and Fernandez 2012).However, this was

10 "The movie opened, at number one, in the midst of an election framed by rightwing Republicans and the powerful Christian Coalition as a referendum on Clinton's morality" (Hoberman 1999). 
disrupted by the first murder, whose victim was a former high school teacher. Matteo Patrick Giovanditto was killed by a young man he had just met hours before and taken to his house and who alleged having killed Giovanditto when he felt sexually threatened (Spindel and Fernandez 2012). Mr. G., as he had been known by his students, was described as being enthused by Disney up to the point that he took several trips a year to Disney theme parks - more often than not, taking several of his male students with him. It transpired in the course of the subsequent police investigation that Giovanditto was not a therapist, as he had made his neighbors believe, and that the young men frequently observed dropping by his house were not his patients. Stories about his too-close-for-comfort relationship with former male students further complicated the picture, making many residents wonder if evil may be lurking beneath the seemingly perfect beauty of Celebration (Spindel and Fernandez 2012).

If Pleasantville ultimately becomes a different place (an unpleasant place, for those who would do anything in their power to maintain the status quo), measures were taken from the very start to ensure that Celebration did not become somewhere where there was little to celebrate. These measures kept a tight control on the appearance of the city, and, thus, rules included that

all visible window coverings must be either white or off-white. A resident may hold only one garage sale in any 12 -month period. A single political sign (measuring 18 by 24 inches) may be posted for 45 days prior to an election. Any activity that "detracts from the overall appearance of the properties" is prohibited including the parking of residents' pickup trucks on the street. (Pollan 1997)

This is not too different from the fictional neighborhood seen in the episode 'Arcadia' (6x13; March 7, 1999) of The X-Files (1993-2002), where Special FBI Agents and protagonists Fox Mulder and Dana Scully move into the seemingly idyllic planned community of The Falls of Arcadia. There, however, things are far from being as idyllic as publicity puts it, since those who dare to defy the all-powerful homeowners' association's rules (including rules such as no basketball hoops, mailboxes painted in the same exact color and no moves allowed after 6 p.m.), mysteriously disappear off the face of the earth, leaving no trace behind. While the show's usual settings include dense forests, rural areas or populous 
cities, by moving the action of this episode to the suburbs, the show was showing that horror can definitely also exist there. Understandably given the show's nature, there is a paranormal element involved - the 'unruly' neighbors are killed by a mudman summoned to life by the president of the homeowners' association, a mythological figure about which he learned in his business trips to Asia. ${ }^{11} Y e t$, the difference here comes from within. In other The X-Files episodes located in the suburbs, the paranormal, disruptive element of daily life came from outside; for instance, in the two-part episode Dreamland (6x03 and 6x04, November 29, 1998 and December 6, 1998), suburban life was altered because of the father's job in the top-secret Area 51. In "Arcadia", however, the supernatural creature is created because of the own neighbors' love for regulations and discipline to control their community, at the expense of those who dare to disagree openly and go against the rules.

A criticism against Pleasantville was that it was too exaggerated (Hoberman 1999), ${ }^{12}$ but is it really so exaggerated in the face of Celebration? The example of Celebration has been copied by a number of later planned communities over the years. The architectural success and financial viability of the Celebration experiment denote that there exists a niche market consisting of thousands of potential customers/ neighbors who aspire to recover the neighborly lifestyle of the Fifties - people who are willing to part with significant amounts of money to do so, too, as well as to comply with rules and regulations that are so detailed and specific that they would put totalitarian regimes to shame.

At the end of Pleasantville, the quintessential happy ending of the Fifties is achieved not once but twice - in Pleasantville, Mary Sue decides to pursue her education, and everybody has changed for the better and become more tolerant in the process. In 1998 David has realized that perfect is merely a word, but not a realistic aspiration

\footnotetext{
${ }^{11}$ This mudman is reminiscent of the Golem in Jewish tradition, already the topic of another The X-Files episode, "Kaddish" (4x15, February 16, 1997).

${ }^{12}$ In spite of its message of tolerance, Pleasantville was also criticized for being a white, heterosexual fantasy, therefore having a limited scope. Additionally, it was accused of being too mild and staying too close to home to be an effective critique of the representations of the Fifties, given that "the film remembers the 1950s struggle over racial equality, and yet black citizens are completely absent from this suburb, and those liberated from Pleasantville's strictures are all white" (Dickinson 2006). Instead, racial matters were hinted at by the soundtrack, since "the music of the 'coloreds' is that of nascent rock and roll and jazz. As characters become 'colored,' the film consistently shifts from 'white' to 'black' music" (Dickinson 2006).
} 
and has come to appreciate his mother all the more, with her flaws and all. He now understands that "nothing went wrong. People change," which applies to Pleasantville as well as the real world. However, as Celebration shows, there are people who still aspire to recreate the Fifties' lifestyle in real life.

\section{WORKS CITED}

Andersen, Kurt. "Pleasantville: Can Disney reinvent the burbs?"The New Yorker Magazine. 6 September 1999.http://www.kurtandersen.com/journalism/ the-new-yorker/pleasantville/ Accessed 2 January 2013.

"Celebration, Florida."Wikipedia, The Free Encyclopedia. 25 November 2012. http://en.wikipedia.org/wiki/Celebration,_FL Accessed 9 January 2013.

Craven, Jackie.“Celebration, Florida.”About.com. n.d.http://architecture.about. com/od/plannedcities/ss/celebration.htm?p=1 Accessed 8 January 2013.

Dickinson, Greg. "The Pleasantville Effect: Nostalgia and the Visual Framing of (White) Suburbia."Western Journal of Communication. 70. 3 (2006). Retrieved from Academic OneFile. Web. Accessed 2 January 2013.

“Epcot."Wikipedia, The Free Encyclopedia. 2 January 2013. http://en.wikipedia. org/wiki/Epcot Accessed 9 January 2013.

Goodnough, Abby. "Disney Is Selling a Town It Built to Reflect the Past." The New York Times. 16 January 2004. http://www.nytimes.com/2004/01/16/ national/16TOWN.html?pagewanted=print Accessed 15 January 2013.

Grainge, Paul. "Colouring the past: Pleasantville and the textuality of media memory."Memory and Popular Film. Ed. Paul Grainge Manchester and New York: Manchester University P, 2003. 202-219.

Hoberman, J. "Review of Pleasantville."Sight and Sound(1999). 49. Web. http://www.tdnet.com/Madrono/resolver/?genre=article\&issn=00374806 $\&$ volume $=9 \&$ issue $=1 \&$ title $=$ Sight + and + Sound $\&$ spage $=14 \&$ date $=19990$ $101 \&$ atitle $=$ Under + the + rainbow\&aulast $=$ Hoberman\%2C $+\mathrm{J}$ Accessed 14 January 2013.

Kahlenberg, Richard. "A Colorful Salute to Reading."Los Angeles Times.11 November 1998. http://articles.latimes.com/1998/nov/11/news/cl-41404 Accessed 4 May 2009.

Pleasantville.Dir. by Gary Ross.Perfs. Tobey Maguire, Reese Witherspoon, William H. Macy, Joan Allen, J. T. Walsh, Jane Kaczmarek. Screenplay by Gary Ross.New Line Cinema, 1998. 
Pollan, Michael. "Town-Building Is No Mickey Mouse Operation."The New York Times.14 December 1997. http://www.nytimes.com/1997/12/14/ magazine/town-building-is-no-mickey-mouse-operation. html?pagewanted=all\&src=pm Accessed 14 January 2013.

Porter, Robert. "Habermas in Pleasantville: Cinema as Political Critique." Contemporary Political Theory(2007). 6: 405-418.

Quart, Leonard and Albert Auster.American Film and Society Since 1945. $2^{\text {nd }}$ edition. Westport, CT and London: Praeger, 1991.

Riddle, Lyn."At Celebration, Some Reasons to Celebrate."The New York Times. 7 March 1999. http://www.nytimes.com/1999/03/07/realestate/atcelebration-some-reasons-to-celebrate.html?pagewanted=print\&src=pm Accessed 15 January 2013.

Spindel, Barbara and Maria Elena Fernandez. "Murder in Celebration, Florida: The Victim's Secret Life."The Daily Beast. 10 September 2012. http://www. thedailybeast.com/articles/2012/09/10/murder-in-celebration-florida-thevictim-s-secret-life.html Accessed 8 January 2013.

Taylor, Charles. "Exile in Pleasantville."Salon. 23 October 1998. http://archive. salon.com/ent/movies/reviews/1998/10/23reviewb.htmlAccessed 30 April 2009.

The X-Files. The Complete Collector's Edition. 20th Century Fox Home Entertainment. 19 November 2007.

"Tour of Celebration."Celebration Town Center, Florida. n.d.http:// celebrationtowncenter.com/tour-of-celebration-town-center Accessed 8 January 2013.

Received: 02 April 2014 Accepted: 03 November 2014 


\title{
CAN LITERARY WORKS HELP TO MEMORIALIZE NATURAL DISASTERS? ZORA NEAL HURSTON'S THEIR EYES WERE WATCHING GOD ${ }^{1}$
}

\author{
Aitor Ibarrola-Armendáriz \\ Universidad de Deusto
}

\begin{abstract}
The wind came back with triple fury, and put out the light for the last time. They sat in company with the others in other shanties, their eyes straining against crude walls and their souls asking if He meant to measure their puny might against His. They seemed to be staring at the dark, but their eyes were watching God. ---Zora Neale Hurston, Their Eyes Were Watching God (236)
\end{abstract}

\begin{abstract}
Although relatively little critical attention has been paid to the closing chapters of Zora Neale Hurston's Their Eyes Were Watching God, the representation of the 1928 cyclone that struck the Everglades in Florida can be seen to have left a profound — and traumatic_impact on both the relations among different human groups and the author's narrative technique. Not only is the title of the book extracted from this section of the book, but faced with the "monstropolous beast" (239) of a Caribbean hurricane, the main characters of the novel realize that interracial attitudes and social structures begin to change shape and lose stability. This article shows that questions of class and race rise in both structural and figurative importance in the closing chapters of the book, and that Hurston was genuinely committed to memorializing the

${ }^{1}$ The research carried out for the writing of this article has been partly funded by the Spanish Ministry of Economy and Competitiveness (code: CSO2011-24804). A shorter version of the essay was delivered at the Conference "Dashed all to pieces': Tempests and Other Natural Disasters in the Literary Imagination,” held at the University of Porto in 2011.
\end{abstract}


losses and mental wounds of those who have generally been forgotten in official records.

Keywords: Natural disasters, collective trauma, race relations, narrative technique, Zora Neal Hurston, Their Eyes Were Watching God.

\section{Resumen}

Aunque, en general, no se ha prestado excesiva atención a los capítulos finales de la novela Sus ojos miraban a Dios de Zora Neale Hurston, su retrato del ciclón que asoló los Everglades de Florida en 1928 muestra el tremendo impacto que el desastre dejó tanto en las relaciones sociales en la región como en la técnica narrativa de la autora. Además de proceder el título de esta última parte del libro, al enfrentarse a la "bestia montruopulosa" (239) de un huracán, los protagonistas se dan cuenta de que las relaciones inter-raciales y las estructuras sociales cambian de forma y pierden su estabilidad. Este artículo analiza cómo cuestiones de clase y raza adquieren mayor relevancia estructural y simbólica en la parte final de la novela, y cómo Hurston sintió la urgente necesidad de representar las pérdidas y las heridas sicológicas de los personajes olvidados en las historias oficiales.

Palabras clave: desastres naturales, traumas colectivos, relaciones inter-raciales, técnica narrativa, Zora Neal Hurston, Sus ojos miraban a Dios.

\section{Introduction: The Psycho-Wounds Resulting from Natural Disasters}

In his groundbreaking and meticulous study Everything in its Path (1976), Kai Erikson defined collective trauma as

... a blow to the basic tissues of social life that damages the bonds attaching people together and impairs their prevailing sense of commonality. The collective trauma works its way slowly and insidiously into the awareness of those who suffer from it, so it does not have the quality of suddenness normally associated with "trauma." But it is a form of shock all the same, a gradual realization that the community no longer exists as 
an effective source of support and that an important part of the self has disappeared. (Everything 153-54)

Erikson's contribution has been essential in establishing the importance of collective traumas in the contemporary world, as opposed to earlier approaches that had generally focused on the examination of individual psychological wounds. His chilling account of the aftereffects that a devastating flood had on the sixteen villages of a small Appalachian community in West Virginia offers significant insights into the community-wrenching consequences of this type of disasters and the profound cultural crises they are likely to produce. Subsequently, Erikson also wrote about a variety of cases in which closely-knit communities had been destroyed by human-induced and technological catastrophes. These disasters proved even more traumatic because, in most instances, they could have been prevented and they seriously disrupted the sense of order necessary for the proper functioning of nature and society. In both types of disasters, it is clear that a great deal of epistemological uncertainty and ontological confusion is generated (cf. Stock and Stott) as the loss of communality causes unpredictable reactions under the altered conditions:

It is the community that offers a cushion to pain, the community that offers a context for intimacy, the community that serves as the repository for binding traditions. And when the community is profoundly affected, one can speak of a damaged social organism in almost the same way that one would speak of a damaged body. (New Species 234; italics in original)

According to Erikson, once that sense of communality is lost, all kinds of troubling questions come up about the status of the self and the laws governing the universe around: Who am I? Why has the world become chaotic? Given these premises, one does not need to stretch one's imagination very much in order to see the remarkable similarities between the Buffalo Creek community in West Virginia and the black agricultural workers in the Everglades, Florida, in Zora N. Hurston's well-known novel. As becomes evident in the second half of Hurston's narrative, the dawning of new economic interests in the region also increased the distance between the socio-cultural poles in what Erikson calls "axes of variation" (Everything 84-88), thus making some of the groups more vulnerable than others. My discussion below will 
demonstrate that farmers and other social categories in the Everglades gradually grew either self-centered or group-centered in rather extreme ways when they were exposed, first, to the immense fertility of the land and, afterwards, to the destructive power of the hurricane. Here is the protagonist's early perception of the land:

To Janie's strange eyes, everything in the Everglades was big and new. Big Lake Okechobee, big beans, big cane, big weeds, big everything. Weeds that did well to grow waist high up the state were eight and often ten feet tall down there. Ground so rich that everything went wild. Volunteer cane just taking the place. Dirt roads so rich and black that a half mile of it would have fertilized a Kansas wheat field. Wild cane on either side of the road hiding the rest of the world. People wild too. (Hurston 193)

As was the case with the mining sites in West Virginia in the late 1960s and early 70s, the agricultural exploitation of this area in Florida was in the hands of people from outside the region who rarely paid much attention to the living and working conditions of the laborers. This fact had very significant implications for the ways in which survivors were able to react to the tropical storm that struck the area in September 1928. It will be observed that the Dantesque scenario left in the wake of the cyclone was characterized by much psychological disorientation, long-lasting signs of social disorganization, and a lack of effective community assistance, either from within or without. ${ }^{2}$ In Arthur Neal's opinion, cataclysmic events such as natural disasters or armed conflicts have "an explosive quality" that is likely to disrupt the extant foundations of the social world so that radical changes may occur in very short periods of time (9-10). The last three chapters of Zora N. Hurston's Their Eyes Were Watching God (1937) can be seen to portray both the heartbreaking images of the hurricane wreaking havoc on a place that up to then had seemed quite paradisiacal and the devastating effects that the flood had on the survivors as they tried to recover, at least partly, from their social and psychological disarray.

Interestingly, to date very little attention has been paid to the hurricane section of the book. Not only that, but some reviewers and

${ }^{2}$ Erikson also concludes his analysis by noting that the disruptive state of community affairs continued in Buffalo Creek at the time he was completing his writing in 1975, three years after the flood (Everything 194). 
literary critics have complained for years that the title of Hurston's novel - which is taken from this section- does not do justice to it or may even be misleading in view of the achievements of the work. ${ }^{3}$ Among those achievements, the following are often mentioned: on one hand, there are the protagonist's recurrent attempts to transcend the roles that a heavily patriarchal and race-conscious society imposes on her; on the other, the use of vernacular speech and African-American storytelling conventions serves the purpose of giving shape and voicing the concerns and feelings of the underprivileged. While admitting that Hurston's strong heroine and her masterful use of the figurative and the dialectal are among her main contributions to the African-American novel, we would commit a serious analytical blunder if we viewed the closing chapters of the novel as just a convenient way of wrapping up these other dimensions of the narrative. The onset of the tropical storm does definitely allow the author to stretch her literary skills in original ways:

Ten feet higher and as far as they could see the muttering wall advanced before the braced-up waters like a road crusher on a cosmic scale. The monstropolous beast had left its bed. The two hundred miles an hour wind had loosed his chains. He seized hold of his dikes and ran forward until he met the quarters; uprooted them like grass and rushed on after his supposed-tobe-conquerors, rolling the dikes, rolling the houses, rolling the people in the houses along with other timbers. The sea was walking the earth with a heavy heel. (239)

Yet, besides the astounding vividness and virtuosity with which Hurston depicts the deadly hurricane - which is presented as a destructive, "senseless monster" (234) — and its aftermath, it is also important to note that the author takes this opportunity to show how social structures and human attitudes change quite dramatically when events of this nature occur. The same as Erikson claimed that observers of the accident in the Appalachian mountains failed to notice the real magnitude of the "great change" that the community underwent (see Everything 71), Hurston also seems to be sending a subtle message to all those critics who have not heeded her intention to represent how human

\footnotetext{
${ }^{3}$ Sheila Hibben, for one, described the title of the book in an early review as "inept enough". Curiously, however, her review is among the most positive assessments of the novel, stressing the author's "warm and vibrant touch," which saves the work from its possible limitations (see Hibben 22).
} 
relations and norms of behavior are deeply transformed by calamitous accidents of this kind. There is no denying that most of the key characters and collectivities in Their Eyes need to rethink their positions regarding others, for new responses and dispositions emerge that would have been unthinkable under other circumstances. As will be seen below, it was the position of the Bahaman and African-American agricultural workers in the Everglades that became particularly vulnerable. Rachel DuPlessis argues that one of Hurston's main achievements is that she compels the reader to carry out a thorough "critical analysis of multiple social determinants in their narrative meanings" (97), and she goes on to remark that this type of analysis becomes especially important in the latter part of the book in which those social determinants - race, class, and gender- are substantially altered by the effects of the hurricane. It will become evident that this close reading and critical dissection of this part of the novel seems especially necessary when there has been a tendency throughout history to neglect the consequences that the catastrophe had for significant segments of the population.

Jeff Klinkenberg published an article in the St. Petersburg Times in September 2008, reporting on a memorial event organized by the township of West Palm Beach to remember the over 2,000 victims of the 1928 hurricane. As the author reminds us, "September 16, 1928, fell on a Sunday. Folks noticed the gray skies on the way home from church. That evening, the storm blasted ashore in southern Palm Beach County, sweeping aside trees and buildings. Then it roared inland toward the state's largest lake" (Klinkenberg). ${ }^{4}$ Despite the dizzying number of casualties and the overwhelming destruction caused by the floods that followed the hurricane, it took over seventy years for the city to purchase the land where the mass graves had been dug and to build a modest memorial park where visitors and relatives could mourn their dead. Incidentally, we also learn in the article that a great majority of the victims of the natural disaster were poor, Bahaman and African-American migrant farmers whose remains were quickly —and rather shabbily - buried to avoid epidemics, thus, Klinkenberg says, adding "insult to injury." The fact that eighty years after the

${ }^{4}$ Eliot Kleinberg also offers all kinds of enlightening details about the arrival and the effects of the hurricane, both on the coastal area and inland (see especially 69-95). He decided to introduce a quotation from Hurston's novel as the epigraph to his scholarly book. 
cataclysm some local authorities and residents were going to take time off to commemorate it is a sign that the psychological wounds inflicted by the disaster are not fully healed as yet. As we know from the literature on the topic, this sign —or claim (Thomson 1998) — refers to a significant injury, a protest against the oblivion and profanation of basic rights, and, ultimately, a demand for some sort of institutional and emotional reparation. Most trauma theorists would agree now that the psychological wounds left by collective disasters are not so much the result of a human group experiencing pain at a particular historical moment but "of this acute discomfort entering into the core of the collectivity's sense of its own identity" (Alexander 10). Indeed, had it not been for the efforts of writers like Hurston who have given new significance to the death of those black laborers, it is difficult to believe that the "trauma process" (Alexander 11) would have been initiated. Moreover, Alexander explains that trauma is "a socially mediated attribution" of meaning to events that would otherwise not have been read as signs of injustice, unequal treatment, and historical erasure (8).

\section{Can Figurative Language and Historical Accuracy Coexist in a Literary Work?}

It has often been noted that Zora Neale Hurston failed to gain the favor of most of her contemporary black male writers because she never believed it was her task to create the naturalist, protest fiction that would explore the nation's historical mistreatment of AfricanAmericans. For authors such as Langston Hughes, Alain Locke, and Richard Wright, Hurston represented "the perfect darkie": a naïve, uncritical, and complacent Negro who mostly wrote to please white audiences. Wright's review of Their Eyes Were Watching God is among the most direct — and, somehow, violent — indictments of her art:

Miss Hurston voluntarily continues in her novel the tradition which was forced upon the Negro in the theatre, that is, the minstrel technique that makes the "white folks" laugh. Her characters eat and laugh and cry and work and kill; they swing like a pendulum eternally in that safe and narrow orbit in which America likes to see the Negro live: between laughter and tears. (17; emphasis in original)

While it is true that it is difficult to picture Hurston in the stark social realist tradition that Wright led or the motive fiction that Locke 
advocated, it is also unfair, as Barbara Johnson contends, to think that her occasional adoption of the "happy darkie" stereotype "exhaust[s] the representational strategies of her writing" (131). In fact, if recent feminist and cultural approaches have proved anything, it is precisely that her work cannot be easily subsumed under convenient labels such as "the folklorist", "the cultural nationalist" or "the sensualist" (McKay 56). ${ }^{5}$ Contemporary black women writers such as Gloria Naylor and Alice Walker have insisted that the feature that best defines Hurston's fiction is its complex nature and its constant aspiration to represent human beings in all their diversity and multi-dimensionality. Most critics would agree that Their Eyes marks the zenith of her writing career because in this late work she managed to keep a balance between her various interests as an anthropologist, a storyteller, an autobiographer, and a social commentator.

Nowhere is it more evident that Hurston succeeded in blending all those different roles in a highly syncretic work of fiction than in her strategic use of different narrative voices. Indeed, although the novel combines the discourses of a formal, omniscient narrator, who uses standard English and is mostly a mere spectator, and the more intimate voice of the protagonist in black dialect, reflecting on her life experiences, it is only fair to admit that the ultimate object of the work transcends these two narrative instances to tell us about a whole culture. In order to do so, the author uses all her resources as an intellectual, a storyteller, and as a female representative of her people to turn Janie Crawford's story into something much larger: a search for a collective black self. Although this task seems very ambitious, Hurston manages to pull us into her protagonist's story by showing a series of highly revealing episodes and images that - despite their discontinuityinvite us to reconstruct Janie's trajectory from being a young, innocent girl to becoming an independent woman, and the increasing influence that her community had on the process. Wolff has rightly explained that "The narrator of Their Eyes Were Watching God is, one might say, a 'big picture talker' as well, who employs lyrical language in order to allow the reader to make a visual and sensual expression of the text" (226).

${ }^{5}$ McKay shows in her provocative article how sophisticated Hurston was at harmonizing and accommodating diverse strands in her fiction so that, eventually, she offers a more complex and holistic vision of black identity. 
The passages of Hurston's novel that most unnerved Wright and company were those in which the author tries to portray her protagonist's awakening to different dimensions of herself, as well as those others in which she attempts to represent in imaginative ways her culture's worldview, values, and mode of expression. Regarding the former, they accused Hurston of showing a romantic streak and an excessive sentimentality that, in their view, was reminiscent of the most damaging examples of Negro "escapism" and double consciousness in literary works. The much-quoted passage in which Janie becomes aware for the first time of her sexuality is a good instance of the high levels of lyricism that the novel reaches when we gain glimpses of the protagonist's inner development:

She was stretched on her back beneath a pear tree soaking in the alto chant of the visiting bees, the gold of the sun and the panting breath of the breeze when the inaudible voice of it all came to her. She saw a dust-bearing bee sink into the sanctum of a bloom; the thousand sister-calyxes arch to meet the love embrace and the ecstatic shiver of the tree from root to tiniest branch creaming in every blossom and frothing with delight. So this was marriage! She had been summoned to behold a revelation. Then Janie felt a pain remorseless sweet that left her limp and languid. (24)

Curiously, the pictures-in-words and fresh metaphors that many of her - mostly male - contemporaries found unpalatable were precisely those that feminist scholars in the 1970s and 80s often retrieved in order to prove Hurston's uncommon imaginative powers. What to earlier analysts might have seemed irreverent details — such as the heroine's hairstyle or the shape of her horizons at different stagesnow became part and parcel of some of the most compelling meanings of the novel. And the same could be said about the author's efforts to represent authentically the popular forms of folk culture which, again, were sometimes read as examples of the simple-mindedness and comic relief that white readers would expect from African-American writers. Thus, neither the protagonist's monologues about the nature of her inner changes, nor the instances of folktales and traditional jokes, should be interpreted as just figurative asides only tangentially related to the story. In Gayl Jones' opinion, "[the] oral tradition enters, complements and complicates character in the use of 'storytelling' or reported scenes to reinforce dramatic ones" (151). As will be seen 
below, the reservoir of images and tropes that Hurston employs in describing the hurricane is not only an affirmation of the uniqueness of her community's perceptions but also a way of granting importance to elements of reality that would otherwise remain unnoticed.

The historical accuracy with which Hurston depicts the arrival and the early effects of the tropical storm can be easily attested by comparing chapters 18 and 19 of the novel to the newspaper reports and some of the historical reconstructions of the disaster that began to appear almost forty years after the event. ${ }^{6}$ One aspect that surprises readers of Hurston's narrative is that the main characters do not seem to take very seriously the first warnings they get about the onset of the hurricane. However, we have learnt years later that while hurricane warnings were properly issued on the coastal line, inland the information had been much more confusing. Although a call to evacuate the low ground near Lake Okeechobee had been made earlier in the day, the storm did not arrive on schedule and many people returned to their homes. Although Janie and her husband, Tea Cake, are advised by some Bahaman friends to abandon the area, they decide to stay in their place exchanging tall tales and throwing the dice with some friends. However, at some point in the night, the world began to rattle and heavy mists started gathering in the west:

It woke up old Okechobee and the monster began to roll in his bed. Began to roll and complain like a peevish world on a grumble. The folks in the quarters and the people in the big houses further around the shore heard the big lake and wondered. The people felt uncomfortable but safe because there were the seawalls to chain the senseless monster in his bed. If the castles thought themselves secure, the cabins needn't worry. Their decision was already made as always. Chink up your cracks, shiver in your wet beds and wait on the mercy of the Lord. The bossman might have the thing stopped before the morning anyway. (234)

It is interesting to note that, although high winds —reaching 140 mph on the ground-had been crossing the region for hours, Hurston

\footnotetext{
${ }^{6}$ Lawrence Will's account of the storm and some of its demolishing consequences in Okeechobee Hurricane and the Hoover Dike (1961) remained for decades the only book-length study of the 1928 disaster. See also Robert Mykle's Killer 'Cane, the Deadly Hurricane of 1928 (2006), which includes numerous interviews with the survivors and their descendants.
} 
should focus her attention on the lake, transforming it into a perverse monster. But, in fact, despite the heavy rain and violent winds, this gigantic mass of water was to prove the most fatal enemy as the southblowing gale was going to cause a storm surge that overflowed and destroyed the dike surrounding the southern shore of the lake. By the time Janie and Tea Cake manage to gather their insurance papers and a few of their belongings, the water is almost up to their waists outside and they have to keep "dodging flying missiles [and other] floating dangers": “... And the lake. Under its multiplied roar could be heard a mighty sound of grinding rock and timber and a wail. They looked back. Saw people trying to run in raging waters and screaming when they found they couldn't. A huge barrier of the makings of the dike to which the cabins had been added was rolling and tumbling forward" (238-39). Hurston describes the lake as "a tired mammoth" that came muttering and grumbling onward, destroying everything in its path. We know now that the resulting flood covered with water an area of hundreds of square kilometers and that in some places the water was over seven meters deep. Telephone poles and power lines were downed by the winds and the water, and as seen in the novel, houses floated off their foundations and, in most instances, were dashed to pieces. As the passage above shows, although Hurston is obviously interested in capturing many of the objective details of the disaster, she also wants to keep a record of the human responses to it, which ranged from sheer astonishment to desperate struggles for survival:

Another man clung to a cypress tree on a tiny island. A tin roof of a building hung from the branches by electric wires and the wind swung it back and forth like a mighty ax. The man dared not move a step to his right lest this crushing blade split him open. He dared not step left for a large rattlesnake was stretched full length with his head in the wind. There was a strip of water between the island and the fill, and the man clung to the tree and cried for help. (244)

It is not surprising that many of the scholarly books on the 1928 hurricane that have recently been published should generally include Hurston as one of the key sources academics consult when trying to get the right picture of what happened in the belt south of lake Okeechobee during those few trying days. Her specific references to the recentlycreated Red Cross, the small armies of mostly black men, and the limited relief assistance that came from other parts of the State are 
evidence of how well-informed and rigorous the author wanted to be in her rendition of the disaster.

\section{Changing Human Relations in the Hurricane Section of Hurston's Novel}

In an article entitled "Differential Disasters", Schwartz has recently studied the 1928 hurricane as a revealing example of "the commonly shared threat of natural disasters [in the Caribbean area] and the differential effects caused by the social, cultural, economic and political contexts of the storms" (4). As this specialist sees it, by looking into the different impact that storms of this kind have in particular places -in the case of his analysis, Florida and Puerto Rico-, we become aware of the ways in which local conditions invariably affect and serve to contextualize the same natural disasters. Although he maintains that both societies showed "a desire to rebuild for the future" (6), it is clear that differences of class and color made that aspiration much more difficult to accomplish in the case of Florida. In fact, the Red Cross felt compelled to create a "Colored Advisory Committee" that had, among other tasks, the object of verifying the "rumors" that the aid was not being equally apportioned among blacks and whites, and that the former were bearing the brunt of the early sanitation and reconstruction work (Cf. Kleinberg 213-46). As Schwartz and other scholars have noted, the socio-cultural and economic contexts in which disasters take place are likely to determine the kind of effects they have on the local populations but, likewise, social dynamics among different human groups are bound to be inevitably altered by traumatic events of this kind. In this sense, it is not surprising to discover that ethnic conflicts, revolts, revolutions or political elections in the Caribbean region have frequently been preceded by one or several hurricanes.

Hurston's closing chapters in Their Eyes Were Watching God provide ample evidence that class, ethnicity, color, and gender are all markers that will define the characters' response to the disorienting events and their perceptions of each other. In DuPlessis' opinion, "Race, class, and color within race rise in structural and figural importance in the latter part of the book, building toward, and away from, the hurricane" (116). Not only that but, as will be seen below, the protagonist's destiny and that of some of the secondary characters is determined by the position 
they occupy in a social hierarchy that becomes more and more apparent as the hurricane sweeps the land. Janie and her husband are exposed to a blatant instance of this kind of prejudicial difference when, after dragging themselves for quite a while in the fill, trying to get away from the chasing lake, they finally reach a more elevated area where they hope to rest: "But it was crowded. White people preempted that point of elevation and there was no more room. They could climb up one of its high sides and down the other, that was all. Miles further on, still no rest" (243). This scene graphically illustrates how much more slippery the social pyramid becomes in times of difficulty and how class and race determinants weigh heavily on the fate of characters. ${ }^{7}$ It is generally assumed that it is the dominant group that demarcates its rights and prerogatives regarding the other collectives, but Hurston seems quite convinced that prejudiced attitudes are not observable only among the privileged. Repeatedly told by the Seminole Indians that they should abandon the low lands because signs of the hurricane's arrival are becoming increasingly evident, this is how part of the black community responded to their advice:

Everybody was talking about it that night. But nobody was worried. The fire dance kept until nearly dawn. The next day, more Indians moved east, unhurried but steady. Still a blue sky and fair weather. Beans running fine and prices good, so the Indians must be wrong. You couldn't have a hurricane when you're making seven and eight dollars a day picking beans. Indians are dumb anyhow, always were. (229)

The second half of this paragraph quite clearly records the train of Tea Cake's thoughts since, as the reader has come to realize in previous chapters, he has grown ostensibly greedy and money-driven when things begin to roll their way "on the muck." The onset of the hurricane will make Janie's husband change in significant ways and major problems will result when his class-conscious and male-chauvinist attitudes aggravated by disease (rabies) — turn him into a rather unrecognizable person: "Janie saw a changing look come in his face. Tea Cake was gone. Something else was looking out of his face" (269). While discussing this change, DuPlessis praises again the great cunning with which Hurston

\footnotetext{
${ }^{7} \mathrm{~A}$ few critics would disagree with this reading of the incident, since they have contended that white people on the Six-Mile Bend are not necessarily demonstrating selfishness by occupying that space. The fact that they do not even allow the blacks to rest for a few minutes on the bridge is revealing, though.
} 
places traps in front of some of the characters that are "built of the cross-currents of class and race, with a tricky admixture of gender and sexuality" (116).

However, it would be rather short-sighted not to notice that Hurston's criticism of how human collectives are transformed by difficult circumstances falls primarily on those who used the disaster to enhance their feelings of superiority and supremacy. In this regard, the most telling episode comes up when the sullen armies of mostly black men recovering the corpses and digging mass graves are told by the guards to examine the victims and to treat them differently depending on their skin color: "Got orders from headquarters. They makin' coffins fuh de white folks. 'Tain't nothin' but cheap pine, but dat's better'n nothin'. Don't dump no white folks in the hole jus' so" (235). Of course, the workers react rather abruptly to this differentiation, not so much because of what it signifies in terms of postmortem unequal treatment, but because of the precious extra time that classifying the corpses would take and the impossibility of determining the color of many of them anyway, due to their degree of deterioration:

The guards had a long conference over that. After a while they came back and told the men, "Look at they hair, when you cain't tell no other way. And don't lemme ketch none uh y'all dumpin' white folks, and don't be wastin' the boxes on colored. They's too hard tuh git holt of right now."

"They's mighty particular how dese dead folks goes to judgment", Tea Cake observed to the man working next to him. "Look lak dey think God don't know nothin' 'bout de Jim Crow law." (253-54)

The irony of Tea Cake's comment remains highly resonant not only because it makes explicit the nonsense of the dominant group's discriminatory practices but, also, because it points at fissures in the social system that, to date, have by no means been completely repaired. In an editorial in USA Today in 2003, Deborah Sharp argued that the effects of the Okeechobee hurricane could still be seen not just in the South Florida landscape but, mainly, in the divisions between blacks and whites. As this journalist points out, even after all these decades, "resentment lingers over a storm recovery effort that was tainted by racism" (Sharp). However, it is important to underline the fact that while the above-mentioned report by the Red Cross and that produced 
by the Florida Chamber of Commerce remained silent about those racist incidents, Hurston's vivid presentation of these events shows some of the most unpalatable consequences of disasters, at least with respect to interracial relations.

\section{Conclusions}

Jeffrey Alexander has argued that "Representation of trauma depends on constructing a compelling framework of cultural classification. In one sense, this is simply telling a new story" (12). Yet, we have also learnt from experience that the symbolic process of putting together that narrative is by no means easy and, in many cases, it may take several generations to take root. I have tried to demonstrate in this article how the contributions of creative writers and journalists are decisive in initiating and bringing to a successful end the construction of that framework of symbolic representation. Both Zora Neale Hurston and Jeff Klinkenberg have done their best to engage in the delicate work of providing meanings for a traumatic event that, like many others, could well have been forgotten or have remained unknowable without their efforts (see Klinkenberg "Storm"). In their work as fiction writer and journalist, respectively, one notes the urgent desire to see the psychological wounds left by a collective disaster finally cured by both restoring memory and trying to lift the societal repression that still lives on in the culture.

I have used the last few chapters from Zora Neale Hurston's Their Eyes to show that this novel is often read through reductive prisms feminists, folklorists, or otherwise - that pay little attention to structural elements that would make us understand her work in a rather different light. While never denying the validity of interpretations that highlight the heroine's courageous attempts to form a self of her own or the magnificent use that the author makes of storytelling conventions and dialect to articulate the concerns and aspirations of her people, I think she reveals other socio-cultural interests in the hurricane section that also deserve our attention. For one thing, we have seen that Hurston seems much more exact and detailed in her descriptions in these chapters, as if she were trying to reproduce as precisely as possible both the onset and the immediate results of the disaster. On the other hand, she seems especially engrossed in showing how unexpected shifts begin 
to take place in human relations and social structures as they had been articulated earlier in the novel. It has become evident in the concluding part of the essay that issues of race and class need to be re-evaluated after we read the closing chapters of the novel, since the disaster will produce significant transformations in how groups and individuals perceive and deal with each other. This re-evaluation is particularly urgent because only by looking into it will we be able to explain with a certain degree of accuracy the kind of neglect that the shocking event has historically received.

\section{WORKS CITED}

Alexander, Jeffrey C. "Introduction: Toward a Theory of Cultural Trauma." Cultural Trauma and Collective Identity. Eds. J. Alexander et al. Berkeley, L.A., and London: University of California Press, 2004. 1-30.

DuPlessis, Rachel. "Power, Judgment, and Narrative in a Work of Zora Neale Hurston: Feminist Cultural Studies." New Essays on Their Eyes Were Watching God. Ed. Michael Awkward. Cambridge and New York: Cambridge UP, 1990. 95-123.

Erikson, Kai T. Everything in its Path: Destruction of Buffalo Creek. New York: Simon \& Schuster, 1976.

----. A New Species of Trouble: The Human Experience of Modern Disasters. New York: W.W. Norton \& Co., 1995.

Hibben, Sheila. "Review of Their Eyes Were Watching God." Zora Neale Hurston: Critical Perspectives Past and Present. Eds. Henry L. Gates and K.A. Appiah. New York: Amistad Press, 1993. 21-22.

Hurston, Zora Neale. Their Eyes Were Watching God. 50th Anniversary Edition with a Foreword by Sherley A. Williams. Urbana and Chicago: University of Illinois Press, 1978 (originally published 1937).

Johnson, Barbara. "Thresholds of Difference: Structures of Address in Zora Neale Hurston." Zora Neale Hurston: Critical Perspectives Past and Present. Eds. Henry L. Gates and K.A. Appiah. New York: Amistad Press, 1993. 130-40.

Jones, Gayl. "Breaking Out of the Conventions of Dialect." Zora Neale Hurston: Critical Perspectives Past and Present. Eds. Henry L. Gates and K.A. Appiah. New York: Amistad Press, 1993. 141-53. 
Kleinberg, Eliot. Black Cloud: The Great Florida Storm of 1928. New York: Carroll \& Graf Publishers, 2003.

Klinkenberg, Jeff. “Unmarked but not Unmourned: 1928 Hurricane's victims get memorial 80 years later.” St. Petersburg Times (14 September 2008). Web. 22 November 2011. <http://www.tampabay.com/features/humaninterest/ article808161.ece>.

---. "A Storm of Memories." St. Petersburg Times (12 September 1992). Web. 22 November 2011. <http://www2-sptimes.com/weather/HG.2.html>.

McKay, Nellie. “'Crayon Enlargements of Life': Zora Neale Hurston's Their Eyes Were Watching God as Autobiography." New Essays on Their Eyes Were Watching God. Ed. Michael Awkward. Cambridge and New York: Cambridge University Press, 1990. 51-70.

Mykle, Robert. Killer 'Cane, the Deadly Hurricane of 1928. New York: Taylor Trade Publishing, 2006.

Neal, Arthur. National Trauma and Collective Memory: Extraordinary Events in the American Experience. Armonk, New York: M.E. Sharp, 1998.

Schwartz, Stuart B. "Differential Disasters: The 1928 Hurricane and the Shaping of the Circum-Caribbean Region." ReVista: Harvard Review of Latin America 6.2 (Winter 2007): 3-7.

Sharp, Deborah. "Storm's Path Remains Scarred after 75 Years." USA Today (4 September 2003). Web. 22 Nov. 2011 <http://www.usatoday.com/weather/ hurricane/2003-09-04-hurricane-usat_x.htm>.

Stock, Angela and Cornelia Stott, eds. Representing the Unimaginable: Narratives of Disaster. Frankfurt am Main, Berlin, Bern, Bruxelles, New York, Oxford, Wien: Peter Lang, 2007.

Thomson, Kenneth. Moral Panic. London: Routledge, 1998.

Will, Lawrence. Okeechobee Hurricane and the Hoover Dike. St. Petersburg, FL: Great Outdoors Publishing, 1961.

Wolff, Maria T. "Listening and Living: Reading and Experience in Their Eyes Were Watching God." Zora Neale Hurston: Critical Perspectives Past and Present. Eds. Henry L. Gates and K.A. Appiah. New York: Amistad Press, 1993. 218-29.

Wright, Richard. "Between Laughter and Tears." Zora Neale Hurston: Critical Perspectives Past and Present. Eds. Henry L. Gates and K.A. Appiah. New York: Amistad Press, 1993. 16-17.

Received: 25 June 2014 Accepted: 28 October 2014 



\title{
“THEIR TRAVELS WERE REAL TRAVELS": HISTORY AND FICTION IN J.M. COETZEE'S “THE NARRATIVE OF JACOBUS COETZE" AND IN EUROPEAN EXPLORATION NARRATIVES IN SOUTHERN AFRICA
}

\author{
María J. López \\ Universidad de Córdoba
}

\begin{abstract}
My aim in this paper is to analyze J.M. Coetzee's early novella "The Narrative of Jacobus Coetzee", included in his first book Dusklands (1974), focusing on the way it points to the inextricability between history and fiction in exploration narratives, and to the impossibility of giving an objective account of truth, free from the constraints of discourse and ideology. In order to do so, I will be paying attention to Hayden White's and Michel de Certeau's respective theoretical contributions on the writing of history, which indeed have many points in common with Coetzee's fictional elaboration of this issue, and to primary exploration narratives Coetzee draws upon, such as Jacobus Coetzee's original 1760 deposition and William Burchell's Travels in the Interior of Southern Africa (1822, 1824).
\end{abstract}

Key words: J.M. Coetzee, “The Narrative of Jacobus Coetzee”, Hayden White, Michel de Certeau, exploration narratives, history, fiction.

\section{Resumen}

Mi objetivo en este artículo es analizar la novella de J.M. Coetzee, "The Narrative of Jacobus Coetzee", incluida en su primer libro, Dusklands (1974), situando en el centro de mi análisis el modo en que dicho relato apunta a la inseparabilidad entre historia y ficción en narrativas de exploración, y a la imposibilidad de contar la verdad de un modo objetivo y libre de las restricciones del discurso y la ideología. 
Para ello, prestaré atención a las respectivas contribuciones teóricas de Hayden White y Michel de Certeau sobre la escritura de la historia, que ciertamente guardan muchos puntos en común con la reconstrucción ficcional que Coetzee hace de esta cuestión, y a narrativas de exploración primarias en las que Coetzee se inspira, tales como la deposición original de Jacobus Coetzee de 1760 y Travels in the Interior of Southern Africa (1822, 1824), de William Burchell.

Palabras clave: J.M. Coetzee, "The Narrative of Jacobus Coetzee," Hayden White, Michel de Certeau, narrativas de exploración, historia, ficción.

In the field of critical and theoretical work on travel writing, a large number of critics have pointed to the inextricability between history and fiction, reality and imagination. David Spurr, in The Rhetoric of Empire, explains that he wishes to show how colonial nonfiction writings "depend on the use of myth, symbol, metaphor, and other rhetorical procedures more often associated with fiction and poetry" (2-3). For Peter Knox-Shaw, travelogues "show how themes which are basic to the fiction (and subject to the usual trafficking of literary influence) belong in the first place to the actual business of coming to grips with the unknown" (3). Jan Borm has argued that the literary is always at work in travel writing, emphasizing "the hybrid nature of the travel book and travel writing, the role of the fictive and the referential" (13). According to Zweder von Martels, fiction, literary tradition, observation and scholarly discovery are inextricable categories in travel writing (xviii), and as Barbara Korte has put it, the element of storytelling in travel writing is closely related to the element of fictionality (10).

The novella "The Narrative of Jacobus Coetzee" is included in J.M. Coetzee's first book Dusklands (1974). The first novella in the book, "The Vietnam Project", deals with a mythographer, Eugene Dawn, writing a report on psychological warfare in the Vietnam War. There are certainly significant parallels between the two sections. As Head argues, "the analysis of US involvement in Vietnam is a prelude to the consideration of Dutch expansionism in eighteenth-century South Africa" (30), which constitutes the focus of "The Narrative of Jacobus Coetzee". However, 
in "The Vietnam Project", there is no engagement with South African exploration narratives — which is why I have left it out of my analysis, whereas it is in the second novella that Coetzee makes his particular contribution to the field of travel writing, emphasizing, like the critics above, the impossibility of making an unequivocal distinction between the writing of history and the writing of fiction. Hence the relevance of the insights and concepts provided by the theorists Hayden White and Michel de Certeau, both of whom, in their concern with history and historiography, have pointed to the operations and tropes shared by the writer of history and of fiction. For de Certeau, influenced by psychoanalysis, "historians can write only by combining within their practice the 'other' that moves and misleads them and the real that they can represent only through fiction" (14), whereas White defines "historical narratives" as "verbal fictions, the contents of which are as much invented as found and the forms of which have more in common with their counterparts in literature than they have with those in the sciences" (82). I would like to argue that in "The Narrative of Jacobus Coetzee" - the South African literary text that probably best illustrates and problematizes the complex relations between history and fiction in exploration narratives dealing with the southern African contextCoetzee's concern with historiography and fictional rewriting of it stands very close to White's and de Certeau's theoretical and philosophical premises.

"The Narrative of Jacobus Coetzee" is made up of different parts through which J.M. Coetzee playfully constructs a rather confusing fictional game in which he presents himself as the mere translator of an edition in Afrikaans made by his father, S.J. Coetzee. Thus, we first find a "Translator's Preface", by J.M. Coetzee; then, the (fictional) narrative itself - the main part of the novella - told in the first person by Jacobus Coetzee, which includes an introduction with no title, the "Journey beyond the Great River", the "Sojourn in the land of the Great Namaqua", and the "Second journey to the land of the Great Namaqua [Expedition of Captain Hendrik Hop, 16 August 1761-27 April 1762]"; the "Afterword", by S.J. Coetzee; and the "Appendix: Deposition of Jacobus Coetzee (1760)", presented as a reproduction of the original deposition that Jacobus Coetzee, an actual ancestor of J.M. Coetzee, related to the Political Secretariat at the Castle of Good Hope in 1760. In terms of thematic content, "The Narrative of Jacobus Coetzee" gives the 
account of the 18th century expedition that this elephant hunter and adventurer made beyond the Great River, focusing on his encounters with the indigenous Khoisan and Namas of the Western Cape.

The motivations and aims that may have lain behind Coetzee's engagement with European explorers in his home country seem to be hinted at in his fictional memoir, Youth (2002), which deals with the period spent by John Coetzee in London in the 60's. As recounted in Youth, John spent much time in the British Library, writing his MA thesis, and ended up reading "memoirs of visitors to the Cape like Dapper and Kolbe and Sparrman and Barrow and Burchell." John pays special attention to Burchell, apparently fascinated by the fact that "Burchell may not be a master like Flaubert or James, but what Burchell writes really happened" (137). Burchell and his men "really lived, their travels were real travels." Hence, if he wants to write a book as convincing as Burchell's, he will have to give to it an "aura of truth" (138).

What interests me about this passage is that in the attention he pays to William Burchell's writing - a 19th century explorer, author of the Travels in the Interior of Southern Africa (1822, 1824), Coetzee emphasizes the element of reality, the fact that Burchell's travels were 'real travels', with the subsequent evocation of truth, whereas his strategy in Dusklands apparently goes in the opposite direction, as he underlines the gap between the historical narrative and reality, undermining the former's pretensions to truth. Borrowing Hayden White's words in his essay "The Fictions of Factual Representation", Coetzee seems to be concerned with "the extent to which the discourse of the historian and that of the imaginative writer overlap, resemble, or correspond with each other" (121). As White has put it, both the writer of a novel and the writer of a history "wish to provide a verbal image of 'reality" and it is this verbal dimension that brings them close to each other:

facts do not speak for themselves, but ... the historian speaks for them, speaks on their behalf, and fashions the fragments of the past into a whole whose integrity is -in its representation - a purely discursive one. Novelists might be dealing only with imaginary events whereas historians are dealing with real ones, but the process of fusing events, whether imaginary or real, into a comprehensible totality 
capable of serving as the object of a representation is a poetic process. Here the historians must utilize precisely the same tropological strategies, the same modalities of representing relationships in words, that the poet or novelist uses. (125)

In his attention to the role of the historian as that of speaking for facts, White puts the emphasis on the tropological manipulation that the historian must carry out, so as to make them fit into a certain discourse. Furthermore this poetic manipulation cannot be seen as separate from an ideological one. As he rejects "the myth of objectivity" (52) in historiography, White highlights the "the importance of the interpretative element in historical narratives" (54). As put by de Certeau, "the signified of historical discourse is made from ideological or imaginary structures" (42). Similarly, in his novella, Coetzee brings the figure of the historian, represented by his father, to the foreground, underlining his interpretative activity together with his ideological motivations. In his "Translator's Preface", J.M. Coetzee significantly points out that S.J. Coetzee's "Afterword" is "drawn from a course of lectures on the early explorers of South Africa given annually by [his] father at the University of Stellenbosch between 1934 and 1948" (55). The reference to these dates is not without significance, since, as Attwell points out, they mark the period of the rise of formal political power of Afrikaner nationalism in South Africa (44). S.J. Coetzee's use of the figure of this 18th century explorer, then, is part of the project of promotion of white, Afrikaner, nationalist, pioneer history, as his "Afterword" makes clear, where he presents Jacobus as one of "the heroes who first ventured into the interior of Southern Africa," "an ancestor and one of the founders of our people" (108).

However, J.M. Coetzee makes the reader immediately alert to the biased nature of S.J. Coetzee's interpretation of Jacobus, by introducing several contradictions between his father's account of the expedition and the fictional narrative he constructs around the figure of this explorer. One of the main differences is that whereas his father underlines the grand and heroic stature of the man - he describes him as an "extraordinary man" (121), riding "like a god" (116), in the narrative he falls ill, having to suffer the humiliation of being taken to the menstruating hut of the Nama village and of having to helplessly depend on his faithful servant Klawer. On the other hand, his cruel and arrogant extermination of the whole Nama village in the second 
expedition of the narrative is absent from S.J. Coetzee's account, where he is referred to as "a humble man who did not play God" (111). S.J. Coetzee's definition of his work as "a work of history" but also "a work of piety" (108) toward his ancestor actually betrays his claims for objectivity, entailing an acknowledgement of the subjective motivations leading him. He finishes his "Afterword" by expressing his hope that he has "succeeded in evoking something of the reality of this extraordinay man" (121, my emphasis), but the reader, as the novella advances, becomes more and more dubious about the possibility of ever getting to know the 'true reality' of Jacobus Coetzee. As the gaps, contradictions, ideological assumptions and manipulations of the different textual versions of the same set of events begin to emerge, the possibility of reaching an objective and unified vision of reality becomes more and more remote. The "myth of objectivity" (White 52) is definitely ruled out.

For White, histories and novels, as verbal artifacts, are indistinguishable from each other, and this is certainly an effect pursued by Coetzee in his composition of "The Narrative of Jacobus Coetzee", which, in its juxtaposition, translation and rewriting of fictional and non-fictional documents, and fictional and non-fictional characters, makes it difficult for the reader, at times, to tell these dimensions apart. In fact, there has been critical confusion around this question, and in particular, around the status of the deposition of Jacobus Coetzee. As Attwell points out, since Knox-Shaw's discussion of Coetzee's sources for his novella, critics had assumed that the deposition was the only authentic historical document in Dusklands (45). However, as Attwell shows, Coetzee also introduces modifications into the original deposition. He is right when he says that Coetzee "omits cordial exchanges from the record and adds desertion" and that "these alterations radically turn the narrative into a game of power" (46). Thus, Coetzee omits passages from the original deposition such as the moment in which Jacobus claims that the nation of the Amacquas "allowed him to journey through their country without any trouble, and to return in like fashion" (Wikar 285), a passage focused a "friendly" exchange of gifts between Jacobus and this people (285) or a reference to one of the Amacquas "who desired to travel hither with him" (289). On the other hand, Coetzee, in the description of Jacobus's return journey, adds that he was "deserted by his servants" (Coetzee, Dusklands 125). 
My point is how these two different combinations of fragments or facts create two very different kinds of accounts or "stories", using Hayden White's term. In the case of the original one, it is one of understanding and cooperation between Jacobus Coetzee and his servants and the native peoples he encounters; in the case of the one included in Dusklands, the different manipulations, and especially the emphasis on Jacobus's being deserted by his servants, hint, on the contrary, at conflict and confrontation. Also, by calling attention to the different facts told in each of the accounts, Coetzee brings to the surface of the text what White calls the "fashioning process" involved in the writing of history, and which involves both positive and negative "distortion": negative, "consisting of the exclusion of facts that might have been included in the representation of the field," and positive, "consisting of the arrangement of events" so as to make them fit into "an integrated pattern of meaning" (111).

J.M. Coetzee probably borrowed the idea of a servant's desertion from Carel Frederik Brink's Journal, which relates the Expedition led by Captain Hendrik Hop following the first journey undertaken by Jacobus Coetzee. In Brink's Journal, generally characterized by an objective tone and a chronological enumeration of facts and events, we come across the following moment: "Among these Little Namaquas who followed us was a Great Namaqua who accompanied the burgher Jacobus Coetse a year ago to Little Namaqualand. Yesterday, when crossing the river with his cattle, he had the misfortune to fall into the stream and was drowned" (30-31). This passage may have been picked up by Coetzee for several reasons. First, for the unusual particular attention it pays to one of the natives. Second, for the potential suspicion it arises: what is presented as an accident may actually have been something different.

In his fictional reworking of this incident, Coetzee turns this servant into a full character in his narrative: he is Klawer, Jacobus Coetzee's faithful servant, who stays by him when all the rest desert him. On their way back from the land of the Great Namaqua, as they arrive at the Great River, we read that "the violence of the current at once snapped the knots that bound us and swept Klawer over the shallows into deep water" (93). However, after the telling of this first version, we find Klawer again by Jacobus's side, and we are given a second version of his disappearance. In this second one, Klawer gets sick and Jacobus ends up abandoning him in the middle of nowhere to go on with his 
way. As he provides two different versions of the same event, thus creating two totally different stories, Coetzee's text exemplifies White's argument that the historian "transform[s] a chronicle of events into a 'history' comprehended by its readers as a 'story' of a particular kind" (63).

This idea of the transformation of events according to one plot structure or another is anticipated by the epigraph chosen by Coetzee for "The Narrative of Jacobus Coetzee", and which comes from Flaubert's Bouvard and Pécuchet: "What is important is the philosophy of history." This sentence comes from a point in Flaubert's novel in which the protagonists pass "from carelessness as to dates ... to contempt for facts" (152). For Attwell, in Dusklands, "the formula emphasizes the fickleness of data and directs attention to the struggle over history" (45). Nadine Gordimer interprets it as signifying the "fatalism" and "detachment borrowed from history" that we see in Jacobus Coetzee (255). I would add that it is revealing to see this statement in the light of the following reflection by Hayden White in his essay "The Fictions of Factual Representation":

Those historians who draw a firm line between history and philosophy of history fail to recognize that every historical discourse contains within it a full-blow, if only implicit, philosophy of history. ... The principal difference between history and philosophy of history is that the latter brings the conceptual apparatus by which the facts are ordered in the discourse to the surface of the text, while history proper (as it is called) buries it in the interior of narrative, where it serves as a hidden or implicit shaping device. (126-127)

Certainly, what Coetzee does in "The Narrative of Jacobus Coetzee" is to pay close attention to "the surface of the text", and to make explicit the "shaping devices" used in colonial history, as we see in the moment of the first encounter between Jacobus Coetzee and the Great Namaquas, a long passage that needs to be quoted in full:

Tranquilly I traced in my heart the forking paths of the endless inner adventure: the order to follow, the inner debate (resist? submit?), underlings rolling their eyeballs, words of moderation, calm, swift march, the hidden defile, the encampment, the gray-beard chieftain, the curious throng, words of greeting, firm tones, Peace! Tobacco!, demonstration of firearms, murmurs of awe, gifts, the vengeful wizard, the 
feast, glut, nightfall, murder foiled, dawn, farewell, trundling wheels, the order to follow, the inner debate, rolling eyeballs, the nervous finger, the shot, panic, assault, gunfire, hasty departure, the pursuing horde, the race for the river, the order to follow, the inner debate, the casual spear in the vitals (Viscount d'Almeida), the fleeing underlings, pole through the fundament, ritual dismemberment in the savage encampment, limbs to the dogs, privates to the first wife, the order to follow, the inner debate, the cowardly blow, amnesia, the dark hut, bound hands, the drowsing guard, escape, night chase, the dogs foiled, the dark hut, bound hands, uneasy sleep, dawn, the sacrificial gathering, the wizard, the contest of magic, the celestial almanac, darkness at noon, victory, an amusing but tedious reign as tribal demi-god, return to civilization with numerous entourage of cattle - these forking paths across that true wilderness without polity called the land of the Great Namaqua where everything I was to find, was possible. (65-66, my emphasis)

Jacobus imagines four different "forking paths" or stories, full of colonial stereotypes and formulae, with the beginning of each one marked with the phrases "the order to follow, the inner debate." The first story is a typically non-confrontational one that emphasizes the negotiation and cooperation between Europeans and natives (the only moment that makes us think of the possibility of a latent and suppressed violence is the reference to "murder foiled"). In the second one, confrontation and violence increase, and culminate in the third story, with a clear tragic plot, as the explorer is dismembered by the natives, whereas the fourth one has a heroic plot according to which the explorer reigns as a demigod among the natives and returns to civilization with plenty of cattle. Through the devising of these different story lines, Coetzee highlights that "history-writing thrives on the discovery of all the possible plot structures that might be invoked to endow sets of events with different meanings" (White 92).

Most critics of Dusklands have called attention to this peculiar passage, which underlines the way in which Jacobus's relation to reality is absolutely mediated by the categories and stories coming from the colonial narrative in which he is inserted. ${ }^{1}$ I would like to add, following

${ }^{1}$ According to Gardiner, at this point, the eighteenth-century Boer has suddenly access to nineteenth-century fictions and twentieth-century jargon, and outlines four paths or scenarios that are amusing reductions of the plots of typical imperial adventure 
White, that the figurative elements and conceptual apparatus that in historical narratives are usually displaced to the interior of the discourse, even if they work as shaping devices, are here brought to the surface. Thus, Coetzee makes it explicit what White has called "encodation", whose effect is "to familiarize the unfamiliar" by making sense of a set of events which appear strange, mysterious or enigmatic "in terms of culturally provided categories, such as metaphysical concepts, religious beliefs, or story forms" (86). In his encounter with the otherness that the world of the Namaqua offers to him, Jacobus attemps to translate that difference into the familiarity of his European intellectual schemas and cultural categories, complicit with the practices and discourses of imperialism.

Furthermore, it is important to note that none of the four outcomes Jacobus devises coincides with the actual development of his encounter with the Namaqua as told in the narrative. In this way, Coetzee underlines the gap between the colonial stereotypes governing Jacobus's discourse and the actual reality he has to face. Thus, the conception of historiography emerging from this novella is close to that espoused by de Certeau, when he asserts that

historiography (that is, 'history' and 'writing') bears within its own name the paradox - almost the oxymoron - of a relation established between two antinomic terms, between the real and discourse. Its task is one of connecting them and, at the point where this link cannot be imagined, of working as if the two were being joined." (xxvii).

Hence, going back to the point of departure of my analysis - the moment in Youth in which Coetzee seems to be hinting at the genesis of Dusklands - it is interesting that he should make an opposition between Flaubert and Burchell — "Burchell may not be a master like Flaubert or James, but what Burchell writes really happened" (137) — two figures present in "The Narrative of Jacobus Coetzee", and that, in principle, embody the opposing poles of fiction (Flaubert) and history (Burchell). However, as we have seen, Coetzee actually

stories of the Haggard/Kipling kind (181). Marais argues that Jacobus Coetzee is internally evoking "the formulaic plot which characterizes frontier writing" (55). Head defines "the characteristic Coetzee style" as "an interrogation of the chosen narrative modes." In the case of Dusklands, it is the "different discourses of imperialism" that are "parodied" (28). 
turns to Flaubert not to make a point about fiction, but to make a point about history. As regards Burchell, the references to this English explorer in "The Narrative of Jacobus Coetzee" — found in S.J. Coetzee's "Afterword" - seem to highlight his faithful approach to reality, as S.J. Coetzee refers to him in order to state objective and scientific facts: "in Burchell we read of a hunter, one Carel Kriege, being pounded into the earth by a maddened bull, at a time when (census of 1798), the adult White male population was 5546" (112); "a bustard (gompou, Otis kori, discovery alleged to Burchell)" (120). Indeed, in his Preface to his Travels, Burchell argues for the objectivity of his account. He claims that his account has been conducted "with a mind free from prejudice, and in the purest spirit of independence," so that it can be "regarded as a faithful picture of occurrences and observations ... the confirmation of which is readily left to every honest and unprejudiced traveller who may hereafter traverse the same ground" (v, V.1). Certainly, Burchell is regarded, among British explorers at the Cape, as "by far the most complete of the scientific botanists" (Benson \& Collony 1597).

However, together with this scientific current, his work is traversed by an important aesthetic dimension, so that it is "a remarkable blend of systematic scientific observation and an inclusive romantic vision" (Cornwell, Klopper and MacKenzie 190). In White Writing: On the Culture of Letters in South Africa, focused on texts by European writers about South Africa, Coetzee analyzes this aesthetic dimension, presenting Burchell as "a man of science" but also as an "amateur painter" (36) that brings the European tradition of the picturesque with him, trying to read the South African landscape according to it (3639). A moment in which Burchell's attempt to read the South African landscape through a projection of his European literary heritage and artistic sensibility is especially obvious when he contemplates the Gariep river for the first time, a view that "realized those ideas of elegant and classic scenery, which are created in the minds of poets, those alluring fancies of a fairy tale, or the fascinating imagery of a romance" (316, V.1). In White Writing, Coetzee analyzes how Burchell continually points to elements such as colour, foliage, light and water, central for pictorial activity, and which turn the landscapes of Africa and England into spaces completely different from each other (42), arguing that "the European eye will be disappointed in Africa only as long as it seeks in African landscapes European tones and shades" (39). 
Coetzee, then, is fully aware of the way Burchell's approach to South African reality - like Jacobus Coetzee's- is mediated by European cultural and discursive categories.

Furthermore, the apparently straightforward identification between Burchell's travels and 'reality' that we find in Youth is problematized, not only because of the aestheticized dimension of Burchell's work -which brings him close to Flaubert- but also because of his activity as naturalist and collector. During his travels in southern Africa, Burchell collected more than 63,000 objects, and discovered many new species, including the zebra named after him as Equus (Quagga) burchelli (Potgieter 591) or as it is commonly known, Burchell's zebra. Burchell is obviously involved in what Pratt has called a "systematizing of nature as a European knowledge-building project that created a new kind of Eurocentered planetary consciousness" (34) during the 18th and 19th centuries. As we read in his Travels the endless lists of objects and species collected and classified by him, we see him engaged in what de Certeau has called "the displacement of the separation between nature and culture": historians deal with physical objects and raw materials, transforming and carrying them from one region of culture (archives, collections) to another (history) (71). In this way, "they 'civilize' nature" (72). Burchell's contribution, then, has to be seen as complicit with the colonial project of European territorial expansion and scientific domination, which explains S.J. Coetzee's appeal to him. The apparently objective and 'unprejudiced' - borrowing Burchell's own term - character of his activity in southern Africa and his account of it cannot be free from cultural and ideological constraints.

To conclude, both the writer of fiction and the writer of nonfictional accounts, such as exploration narratives, "wish to provide a verbal image of 'reality" (White 122), and that seems to be the desire of the young John Coetzee, in Youth, when, in London, he is struggling to become a writer. Near the end of this work, he tells the reader about a revelation that very much determined his conception of the relation between "verbal images" and "reality": the discovery of Samuel Beckett's Watt. Samuel Beckett, on whom Coetzee wrote his 1969 thesis on stylistic analysis, is probably the single most important literary influence on Dusklands, particularly Beckett's obsession "with form, with language as self-enclosed form" (Coetzee, Doubling 393). The mode of composition and the textual strategies of "The Narrative of Jacobus 
Coetzee" are very much influenced by a linguistic determinism that the early Coetzee inherited from Beckett. The different and contradictory versions of the same events and the manipulation and combination of narrative fragments that we find in this work very much recall the mode of composition in works by Beckett such as Watt or Mercier and Camier, and emphasize the mediation of reality that all verbal artifacts -whether historical or literary, fictional or non-fictional- always entail. Hence the blurring of boundaries between history and fiction; hence the appeal to Flaubert, the novelist but also the historian, and to Burchell, the scientist but also the artist.

In the link it makes between European colonial practices and discourses in southern Africa in the 18th and 19th centuries, and US presence in Vietnam in the 20th century, which we see in the first part of the book, Dusklands shows the pervasiveness of imperialism and its ideological assumptions. By putting the emphasis on the manipulation of facts and the similar discursive strategies that explorers, historians and writers like himself share, Coetzee inserts himself in a long history of Western domination in the southern African context, calling attention to issues of complicity and guilt, while at the same time denouncing the ideological implications of those strategies. As opposed to what Burchell seemed to believe, the writer is never "free from prejudice," nor can s/he write "in the purest spirit of independence" (v, V.1)

\section{WORKS CITED}

Attwell, David. J.M. Coetzee: South Africa and the Politics of Writing. Berkeley: University of California Press, 1993.

Benson, Eugene and L. W. Conolly. Encyclopedia of Post-Colonial Literatures in English, V. 2. London: Routledge, 1994.

Borm, Jan. "Defining Travel: On the Travel Book, Travel Writing and Terminology." Perspectives on Travel Writing. Ed. Glenn Hooper and Tim Youngs. Aldershot: Ashgate, 2004. 13-26.

Brink, Carel Frederik. The Journals of Brink and Rhenius being the Journal of Carek Frederik Brink of the Journey into Great Namaqualand (17612) made by Captain Hendrik Hop and The Journal of Ensign Johannes Tobias Rhenius (1724). Cape Town: The Van Riebeeck Society, 1947. 
Burchell, William J. Travels in the Interior of Southern Africa, Vol. I. London: Longman, Hurst, Rees, Orme and Brown, 1822.

---. Travels in the Interior of Southern Africa, Vol. II. London: Longman, Hurst, Rees, Orme and Brown, 1824.

Coetzee, J.M. Doubling the Point: Essays and Interviews. Ed. David Attwell. Cambridge: Harvard University Press, 1992.

---. Dusklands. London: Vintage, 2004.

---. Youth. London: Secker and Warburg, 2002.

---. White Writing: On the Culture of Letters in South Africa. Johannesburg: Radix (in association with Yale UP), 1988.

Cornwell, Gareth, Dirk Klopper and Craig Mackenzie. The Columbia Guide to South African Literature in English Since 1945. New York: Columbia University Press, 2010.

De Certeau, Michel. The Writing of History. Trans. Tom Conley. New York: Columbia University Press, 1988.

Flaubert, Gustave. The Complete Works of Gustave Flaubert: Embracing Romances, Travels, Comedies, Sketches and Correspondence. Vol. 9. Ed. Ferdinand Brunetière. New York: M.W. Dunne, 1904.

Gardiner, Allan. "J.M. Coetzee's Dusklands: Colonial Encounters of the Robinsonian Kind.” World Literature Written in English 27.2 (1987): 17484 .

Gordimer, Nadine. Telling Times: Writing and Living, 1954-2008. London: Bloomsbury, 2010.

Head, Dominic. J.M. Coetzee. Cambridge: Cambridge University Press, 1997.

Knox-Shaw, Peter. The Explorer in English Fiction. Houndmills: Macmillan, 1987.

Korte, Barbara. English Travel Writing: From Pilgrimages to Postcolonial Explorations. Houndmills: Macmillan, 2000.

Marais, Mike. "Ominpotent Fantasies' of a Solitary Self: J.M. Coetzee's "The Narrative of J.M. Coetzee"." Journal of Commonwealth Literature 29.2 (1993): 48-65.

Martels, Zweder von, ed. Travel Fact and Travel Fiction: Studies on Fiction, Literary Tradition, Scholarly Discovery and Observation in Travel Writing. Leiden: Brill, 1994.

Potgieter, D.J. Standard Encyclopedia of Southern Africa, V. 2. Cape Town: Nasou, 1975.

Pratt, Mary Louise. Imperial Eyes: Travel Writing and Transculturation. London: Routledge, 1992. 
Spurr, David. The Rhetoric of Empire: Colonial Discourse in Journalism, Travel Writing, and Imperial Administration. Durham: Duke University Press, 1993.

White, Hayden. Tropics of Discourse: Essays in Cultural Criticism. Baltimore: The John Hopkins University Press, 1978.

Wikar, Hendrik Jacob. The Journal of Hendrik Jacob Wikar (1779) with an English Translation by A.W. Van Der Horst and the Journals of Jacobus Coetsé Jansz (1760) and Willem van Reenen (1791) with an English Translation by Dr. E.E. Mossop. Cape Town: The Van Riebeeck Society, 1935.

Received: 09 September 2014

Accepted: 25 November 2014 



\title{
VENGEANCE DEFERRED: CHILDREN IN THE SELECTED SHORT STORIES OF SAKI
}

\author{
Sean Moran \\ University of Gdansk
}

\begin{abstract}
This article examines the position of privilege allotted to children in selected short stories by 'Saki' (H.H. Munro 1870-1916) and the possible autobiographical justification behind it. While other writers, then and now, have favoured the child over adults, Saki is notable for the intensity of his bias, by which children are intrinsically cruel but honest, and uncontaminated by the hypocrisy that marks the grown-up. Their behaviour can, however, transgress accepted moral boundaries, especially in their treatment of very young infants.

Saki's fiction is also striking in its lack of mothers, particularly a lack of caring mothers. In their stead there is the older woman who embodies all that is false and callous in the adult world and who, on a significant number of occasions, is the target of vengeance from her young wards. Equally significant is the bond forged between children and animals in an alliance against these representatives of insipid adulthood.
\end{abstract}

Key words: Munro, Saki, short stories, child, animal

\section{Resumen}

Este artículo examina la posición de privilegio que se concede a los niños en las historias cortas selectas de 'Saki' (H.H. Munro 18701916) y la posible justificación autobiográfica que podría abonar dicha postura. Aunque que otros escritores, tanto de antes como de ahora, han privilegiado a los niños sobre los adultos, Saki es digno de notar por la intensidad de su sesgo, según el cual los niños se presentan 
como intrínsecamente crueles pero honestos y no contaminados por la hipocresía que caracteriza a los adultos. Su comportamiento puede, sin embargo, transgredir los límites morales comúnmente aceptados, especialmente en lo que se refiere al tratamiento que prodigan a los más pequeños.

La ficción de Saki sorprende también por la ausencia de madres, en especial de madres cariñosas. En su lugar aparece la mujer mayor personificando todo lo que es falso e insensible en el mundo de los adultos, constituyéndose, en un número significativo de casos, en el blanco de las venganzas de sus jóvenes pupilos. Igualmente significativo es el vínculo que se establece entre niños y animales en alianza contra los representantes de una adultez insípida.

Palabras claves: Munro, Saki, historias cortas, niño, animal

One should normally be wary of identifying an author with his narrative voice/s, but in the case of Hector Hugh Munro ('Saki'), it is not only justifiable but in many ways essential in order to fathom the mind that could create some of the wittiest, most urbane and yet most macabre short stories in the English language. Although, as Ruth Maxey has stressed (2005:9), Saki's children usually promote positive responses in adults, the intention of this article is to examine moments of conflict in a selection of short stories in light of the biographical revelations of the author's sister in an essay published in the 1929 edition of The Square Egg, and Other Sketches. Three of these texts are amongst his best-known work and, significantly, each features a malevolent older, female relative. "Gabriel-Ernest" (Reginald in Russia, 1910), apart from being a disquieting tale of lycanthropy is on a broader scale "a clear suggestion ... of a savagery underlying polite exteriors" (Malcolm, 2012: 148) which may have its roots in the underprivileged childhood experiences of its author. "Sredni Vashtar" (The Chronicles of Clovis, 1912), despite its well-deserved status as one of Saki's most famous works, is also one which is tinged with undeniable biographical references that serve to intensify the enmity between child and adult. "The Lumber Room" and "The Story-Teller" (both from Beasts and Super-Beasts, 1914) point to the fact that Saki's intolerance of the adult world may derive in part from its lack of capacity for phantasy, a quality 
which may have helped the young Munro to survive as a child. As his childhood will be considered as a source of inspiration, it may be useful to differentiate between the personae of Munro as individual and as writer, which the adoption of a pen-name suggests he himself wished to do. The names 'Hector' and 'Munro' will therefore be used in connection with the former, and 'Saki' with reference to the latter.

While Saki bears a certain degree of similarity to Lewis Carroll, Rudyard Kipling or Oscar Wilde, his style is singularly his own, and even a writer like Roald Dahl, renowned for his "anti-authoritarian complicities between children and animals" (Maxey, 2005:2), does not relish the cruelty to such an extent. Part of this is can be put down to the pervasive violence of the age, a "terrible wrongness" which a modern, more sanitised society may at times find unpalatable (Dyson, 2006:vii). In J. M. Barrie's Peter Pan, for instance, there is a group of "lost boys ... children who fall out of their prams when nurse is looking the other way" who are then sent "far away" to the Never Land if not reclaimed within the week (Barrie,1928:32); Peter considers himself abandoned by his mother and warns that "every time a child says 'I don't believe in fairies,' there is a fairy somewhere that falls down dead" (ibid. 31). While modern sensibilities may be slightly perturbed by this apparent insensitivity, Barrie leaves off here. Saki, it is tempting to think, would have probed the manner of the fairies' demise a little more eagerly.

Although Barrie also places children in a privileged position, the difference in outlook can be observed in a scene from Saki's first novel, The Unbearable Bassington, when the eponymous hero argues that Barrie "had a wonderful and tender insight into the child mind and knew nothing whatever about boys" (579). What boy worthy of the name, he asks, would happily play in a cave "when there were wolves and pirates and Red Indians to be had for the asking on the other side of the trap-door?" (ibid.). For Sandie Byrne, the criticism underscores "the sentimentality (especially in relation to mothers), whimsicality, and incipient heterosexual stirrings of tenderness for the female that Saki's stories avoid" (2007: 211). Saki was not only a child-lover, but in many ways a child himself "with all the imagination, the irresponsibility and the harsh cruelty of children developed in him" (2007:139), and A. J. Langguth writes that Saki never let "the restraints of the adult mind filter out the primitive streak that quickens his beautifully crafted stories" (219). 
A. A. Milne, a great admirer, commented on the bizarre elements so commonplace in Saki's work, contrasting them with the world inhabited by his peers: "While we were being funny, as planned, with collar-studs and hot-water bottles, he was being much funnier with werwolves [sic] and tigers" and that "strange boyish insensitiveness of his" (Milne,1926: n.p). Another friend, the journalist, H.W. Nevinson, sees in his characters the same cynicism he detected in the face of the author himself and again revels in the "delightful and ingenious wickedness" (Nevinson, 1930: ix). In Langguth's view, Saki's great skill lies in diluting this malevolent intent with a hefty dose of wit, which subverts the rebellion and ultimately upholds a universal order in its place (1982:173).

If a distinction is made in Saki's fiction between adults and children, then another subdivision exists between the child and the infant, one that Langguth interprets as being essentially sexual in nature (155). A friend of Saki quotes him as saying that "There is one thing I care for and that is youth" (Reynolds,1919:10), and young nubile boys certainly held an attraction for the homosexual Munro that appears in both his fiction and his letters to Ethel.

The homo-erotic fascination of Gabriel-Ernest is unequivocal and David Malcolm discerns phallic undercurrents in "Sredni Vashtar". Small infants like the Toop child, however, are not only asexual but are barely human (in this instance even deprived of a name and gender and referred to merely as "it" or "child"), so the reader is not invited to invest sympathy. Nor indeed is it demanded of the fictional mother, who is expected not to protest as she "had eleven other children" to recompense for the loss, as if the death of one child could be coldly calculated against the survival of others (69). Similarly, in "The Elk", the animal's owner refuses a mother's demands that she have it put down for attacking her young son because "she had eleven children and I had only one elk" (361), and in "Esmé", a gypsy child snatched and eaten by a hyena is described in the same dispassionate manner as the Toop child.

The portrayal of animals in Saki's fiction would easily merit a study of its own ${ }^{1}$. They are often employed as allies to children, or work

\footnotetext{
${ }^{1}$ See, for example, Joseph S. Salemi, "An Asp Lurking in an Apple-Charlotte: Animal Violence in Saki's The Chronicles of Clovis."
} 
as instruments of their will, holding the hope that the tedium of the grown-up world can be defeated, even if temporarily (Byrne, 2007: 217). In "Gabriel-Ernest" this union is personified in the figure of the werewolf protagonist, the physical fusion of the two, who asserts his superiority by outwitting adults and the literal consumption of an infant ${ }^{2}$, while the quasi-divine ferret in "Sredni Vashtar" is a more empowered extension of the weak and frail Conradin.

In "The Penance" the alliance extends beyond death. Three children demand humiliating restitution from a neighbour for the death of their pet cat by threatening to roast his two-year-old daughter over a fire or feed her to pigs. In defence of their actions, they make it clear that they view the cat and the infant on equal terms, whereby the only commensurate reparation for the killing of a cat is the death of a child. Given the circumstances of their upbringing, is not difficult to see Hector, his brother and sister in the figures of the three children, "a girl and two boys" (423), cut off behind a wall, and with parents resident abroad, nor in descriptions of "human hate, impotent yet unyielding, raging yet masked in silence" (ibid.).

Generally, animals "represent what human beings would be like without the veneer of etiquette and social grace - in fact, what human beings really are beneath the surface of upper-class manners, bourgeois respectability, and feigned solicitude for others"(Salemi, 1989:426). They are elemental forces that work independently of, and usually in conflict with humanity and which, like Gabriel-Ernest, intrude directly into the cosy sanctity of the home. Thus, a painter is inspired to paint works such as $O x$ in a Morning Room, Late Autumn progressing to Barbary Apes wrecking a Boudoir ("The Stalled Ox"), and similar imagery is extended to the larger world when another artist makes a name for himself by works with foreboding titles such as Giraffes drinking at the Fountain Pools, Trafalgar Square or Vultures attacking dying Camel, Upper Berkeley Street, suggesting that animals will eventually inherit the relics of human civilisation ("On Approval").

In the three main stories considered here, the child's only retreat is his aptitude for such bizarre invention - a domain to which he can retire to challenge the role as victim. Imagination allows for the existence of

${ }^{2}$ In Saki's first published story, "Dogged" (1899), an adult is metaphorically consumed by an overbearing pet dog. See Dyson, 2006: 3-10. 
inhumanity and death, while the wearisome adult view tries to smother the elemental struggle of life with a layer of sentimentality, deceit and downright lies. This threadbare world is parodied, for example, in "The Reticence of Lady Anne". A husband's attempts to bury the hatchet after a domestic disagreement fail, not because his wife wins the discussion - there is none - but simply because she has been dead for two hours, and he has been too short-sighted to perceive this. The adult world, Saki seems to be saying, is so moribund that life is no longer distinguishable from death. By contrast, the best stories for children incorporate the essential and indisputable brutality of life and recognise death as a component of it — the theme of "The Story Teller."

In an attempt to explain his preference for children over adults, it would be beneficial to look back on Munro's own childhood, as it offers a wealth of traumatic formative influences. Unfortunately, the little information that survives is riddled with uncertainties or unsubstantiated family legend ${ }^{3}$, but despite this parallels emerge which cannot be readily dismissed. In one early incident worthy of his own pen, a great-great uncle was fatally mauled about the head and shoulders by a tiger in India (possibly while the victim was urinating against a bush). Since this incident was immortalised by the Staffordshire porcelain factory with immense commercial success and the figure became a common one in British homes, it is more than likely that the young Hector would have been familiar with it. Later, his mother, after being repatriated from Burma to avoid the hazards associated with pregnancy (her fourth), was charged by a runaway cow in an otherwise quiet English country lane, resulting in a miscarriage which claimed her life as well as that of her unborn child.

As his widowed father remained in Burma, the three remaining children, Charles, Ethel and Hector, were entrusted initially to a grandmother and two paternal maiden aunts with "old-fashioned Scottish ideas of discipline" (Reynolds,1919: xi). Here, according to Ethel, they learnt to conceal their feelings, as any expression of emotion seemed to provoke a perverse amusement in their Aunt Augusta. Stern Victorian ideas on health dictated that the house be kept in darkness, windows "shut and shuttered", allowing only stale air to circulate, and the gardens surrounded by tall walls and a hedge (Munro, 1929:4).

\footnotetext{
${ }^{3}$ These biographical details are taken from Byrne (2008) and Langguth.
} 
Thus the children grew up in an atmosphere that on both the physical and psychological level can genuinely be described as stultifying.

Apart from the physical isolation from their peers that living in the countryside entailed, the spiteful withdrawal of their few existing pleasures on the slightest provocation forced the children to fall back on each other and their fertile, if not exuberant, imaginations. Ethel's first memory of her younger brother, for instance, is one of him chasing his siblings round the nursery table brandishing a flaming hearth brush and shouting "I'm God and I'm going to destroy the world!" (Munro,1929:3). The only relief from the tedium came through rare visits by their father or uncle and the constant presence of animals, including a rook, various "[t]ortoises, rabbits, doves, guinea-pigs, and mice," but primarily cats and hens and cockerels (Munro,1929: 9). The young Hector also took to drawing, usually a succession of images of people being mauled or eaten by wild animals - a sinister foretaste of his fiction.

\section{Boy and Beast}

"Gabriel-Ernest" explores the consequences of disregarding the inherent cruelty of nature. It opens unambiguously with the sentence "There is a wild beast in your woods" (63) - the first of several warnings that are glossed over. Saki shows an unequivocal preference for the infinitely more intriguing and compelling animal/boy over the effete and superficial Van Cheeles, and his language accentuates his bestial nature from the outset: He has a "tigerish gleam" (64) and "tigerish yellow eyes" (65), and his very nakedness strips him of any vestige of a civilised outer layer. With brutal candour, he admits his own duality when prompted to explain his activities:

"I don't sleep at night; that's my busiest time" (64).

"At night I hunt on four feet" (65).

"I don't fancy any dog would be very anxious for my company, especially at night" (ibid.).

His most revealing comment, however, is given in response to an enquiry over his diet:

"What do you feed on?" he [Van Cheele] asked.

"Flesh," said the boy, and he pronounced the word with slow relish, as though he were tasting it. 


\section{"Flesh! What flesh?"}

"Since it interests you, rabbits, wild-fowls, hares, poultry, lambs in their season, children when I get any; they're usually too well locked in at night, when I do most of my hunting. It's quite two months since I tasted child-flesh" (64-65).

These candid admissions draw the reader's attention back to the opening sentence and confirm that the boy possesses an aspect darker than his graceful façade would suggest or that Van Cheele or his aunt ${ }^{4}$ are prepared to accept. During her first encounter with the wild and naked boy in her morning-room, he appeals to her "as warmly as a stray kitten or derelict puppy would have done" (67) and she despatches a messenger to obtain clothing. Once dressed and groomed, he is christened Gabriel-Ernest - "nice suitable names" for such a "sweet" youth (ibid.).

Part of Gabriel-Ernest's success as a man-eater is due to the failure of adults to recognise his savage nature or to react to that nature once suspected. Van Cheele's knowledge of birds and the possession of one stuffed specimen have led his aunt to view him as something of a naturalist, but his familiarity is restricted to harmless, tameable (or dead) fauna. The reaction of the family pets, however, when GabrielErnest appears in the Van Cheele home is more perceptive. The canary is reduced to "frightened cheeps" and the pet spaniel flees, this despite the boy's apparent human form (67). Animals are thus seen as wiser creatures, more in touch with the dark side of nature and more alert to its potential for evil, a side Van Cheele's aunt blithely overlooks in her misplaced Christian philanthropy. She treats the feral 'amnesiac' with thoroughly unwarranted trust and tenderness, names him partially after an archangel ${ }^{5}$, provides him with a superficial veneer of godfearing respectability (his clothes are sent from the Rectory) and in the end naïvely allows him to escort the Toop child home.

This combined failure on the part of aunt and nephew contributes as much to the child's death as Gabriel-Ernest's unabashed honesty over a true character he makes no attempt to conceal. The aunt, still

${ }^{4}$ Unlike some of her kind in Saki's work, she is dignified with a name - Miss Van Cheele, presumably the sister of Van Cheele's father and therefore in precisely the same relationship as Munro to his own guardian aunts.

${ }^{5}$ The Ernest part may well be a reference to Oscar Wilde's The Importance of Being Ernest and the werewolf's physical transformations a less genteel form of 'Bunburying'. 
blinkered by Christian charity, compounds her naïvity by mourning "the lost foundling" and having a plaque erected in the local church to "Gabriel-Ernest, an unknown boy, who bravely sacrificed his life for another" (69), adding obstinate trust to her list of vices.

There are few writers bold enough to contemplate child cannibalism as a plot device, and even fewer who could successfully carry it off as the macabre comedy which "Gabriel-Ernest" undoubtedly is: the sarcastic jibes at Van Cheele's knowledge of the animal kingdom are typical Saki, as is Van Cheele's rejection of a "Gabriel-Ernest is a werewolf" telegram as "a hopelessly inadequate effort at conveying the situation" (68) and Langguth calls the attempt to hide the boy's genitalia with a copy of the Morning Post "unashamed farce" (161). He summarises the entire story as an amalgam of "the erotic, the horrible and the comic" in which, to Saki's credit, the comic manages to triumph (160). It nevertheless retains a distinct underlying hint of animosity towards the predominant Christian morality of the age.

The same hypocrisy displayed by Miss Van Cheele is witnessed in what is probably ${ }^{6}$ Saki's most anthologised story, "Sredni Vashtar." Mrs DeRopp, ten-year old Conradin's cousin and guardian, is an uncaring woman, who cannot admit to herself that thwarting her ward in everything he does is not "particularly irksome" (136). Conradin's loathing of her is in contrast constant, heart-felt and intense, albeit well-concealed. To transcend his loneliness, he has invested all his faith into the figures of a Houdan hen and a small ferret, which he names Sredni Vashtar and worships in a secret hide-out in a dark corner of the garden shed. Condemned by the medical profession to a brief and lonely life, Conradin would have long succumbed to the illnesses which torment his physical body, were it not for the invigorating energy embodied in Sredni Vashtar.

The "lithe, sharp-fanged beast" (137) attains the status of a god "who laid some special stress on the fierce impatient side of things, as opposed to the Woman's religion, which, as far as Conradin could observe, went to great lengths in the contrary direction" (138). The cousin's Christianity belies her harsh and hypocritical regime over Conradin, whose own unique form religion is depicted in terms of the

6 "The Open Window" may be the only rival to the claim. 
"[r]ed flowers", "scarlet berries" (137) and prayer offered up to Sredni Vashtar. This foreshadows the blood that will conclude the story:

Sredni Vashtar went forth,

His thoughts were red thoughts and his teeth were white.

His enemies called for peace, but he brought them death.

Sredni Vashtar the Beautiful. (139)

Once Mrs DeRopp decides to investigate the garden shed and discovers the hen which shares Sredni Vashtar's shrine, Conradin realises that his fantasy is nearing an end. The hen is sold and the callous announcement is made in full expectation that rage and protest will ensue, only to be countered by Mrs DeRopp's sound reasoning and logic. Instead, Conradin holds his tongue and returns to "the world he hated" (139) where "Mrs DeRopp was the ground plan on which he based and detested all respectability" (138).

His prayers to the ferret-idol are eventually answered. Suspecting that the hen was not the only resident, DeRopp ransacks Conradin's bedroom for a key (even privacy is denied him) and returns to the shed. Convinced that his guardian will soon emerge triumphant with "a simple brown ferret in a hutch" (139), Conradin begins to lose faith and resigns himself to being tormented until he proves the doctor right and dies young, since his loss of faith and imagination can only end in death.

It is not Mrs DeRopp who emerges from the shed, but the ferret "dark wet stains around the fur of jaws and throat" (140). As she meets her death in the form of a condensed metonym for all the malice that she denies in the world, Conradin enjoys the unaccustomed luxury of independent decision-making by indulging in "the hitherto forbidden fruit of toast" (Malcolm, 2012: 213).

Bearing in mind the intensity of Conradin's hatred, there is no place for humour in "Sredni Vashtar". Instead, the reader is persuaded of the justice of DeRopp's death and moral judgement is suspended within the "safe parameters of a consciously fictional pseudo-nursery story narrative" (Maxey, 2005:3). By making Conradin such a weak child, Saki clearly expects reader sympathy to lie with him. The relentlessly negative portrayal of DeRopp (an aunt in all but name) and her ability to exert such a detrimental influence over Conradin are contrasted 
with his helplessness and his need firstly to imagine, and then to exact, revenge with the aid of another vulnerable creature. For Byrne, the narrative voice is "not neutral or equivocal" but sides very strongly with the perceived victim (2008:162), and David Malcolm remarks on the discernible distance between Conradin as child protagonist and the adult language used to describe him and to enter DeRopp's mind in an effort "to explain her almost disinterested intent to crush her ward" (Malcolm, 2012: 212).

From the autobiographical point of view, this distance could be perceived as the mature writer looking back on his own past in order to reconstruct it to his advantage - the death of Mrs DeRopp becoming a belated and vicarious triumph over the aunts who had blighted his childhood. Without explicitly referring to their domineering presence, Salemi is still able to sum the story up as "a thoroughly disturbing piece, a textbook case-history of the revenge of the repressed unconscious" (427). The young Hector was likewise a frail and sickly child who was not expected to reach maturity, and his sister reveals that he possessed a Houdan hen, which was put down on the orders of his guardians to save the bother and expense of veterinary treatment (Munro,1929: 9). Thus, "Sredni Vashtar" could with some legitimacy be viewed as vengeance deferred. Where the powerless child cannot give sufficient expression to his anger, the resentment is nurtured internally until such times as the adult can wield the pen to create the "agent of Nemesis" (Byrne, 2008: 127 et passim) in the shape of a ferret which finally redresses the injustice and relegates the factual aunt to the position of inferiority occupied by adults in Saki's fictional hierarchy.

Ethel Munro's uncompromising description of her aunt adds some weight to the idea that Munro "learned [...] to humiliate his oppressors in a dozen ingenious ways on the printed page" (Langguth, 1982: 27).

The other aunt, Augusta, is the one who, more or less, is depicted in "Sredni Vashtar" ... a woman of ungovernable temper, of fierce likes and dislikes, imperious, a moral coward, possessing no brains worth speaking of, and a primitive disposition. Naturally the last person who should have been in charge of children. (Munro, 1929: 7)

As a young child, Munro may have been forced to suffer endless indignities in silence, but as 'Saki' he found himself endowed with the 
means to "send Augusta forth to be devoured by beasts" (Langguth, 1982: 27).

\section{Imagination Stifled}

As in the case of the young Munro, Conradin's realm of fantasy is clearly his only consolation in life, and by the conclusion of the story it has brought him salvation through a symbolic and actual victory over the "necessary and disagreeable and real" world of the adult (136). The essential difference between the two worlds is encapsulated in the description of the Olympic Toy Emporium with which the story "Morlvera" begins.

It was happily named Toy Emporium, because one would never have dreamed of according it the familiar and yet pulse-quickening name of toyshop. There was an air of cold splendour and elaborate failure about the wares that were set out in its ample windows; they were the sort of toys that a tired shop-assistant displays and explains at Christmas time to exclamatory parents and bored, silent children. The animal toys looked more like natural history models than comfortable, sympathetic companions that one would wish, at a certain age, to take to bed with one, and to smuggle into the bath-room. The mechanical toys incessantly did things that no one could want a toy to do more than half a dozen times in its life-time; it was a merciful reflection that in any right-minded nursery the lifetime would certainly be short. (691)

The "elegantly-dressed" doll at the centre of the story is purchased as a birthday gift in an insincere gesture of reciprocation. Even here, the reluctant buyer still manages to outmatch his mother (in keeping with his name - 'Victor'), by having the ingenuity to throw the doll surreptitiously under the wheels of the carriage as he departs. It is crushed with "a soft, pleasant-sounding scrunch" leaving only "a sorry mess of petrol-smeared velvet, sawdust, and leopard skin" (495). In other stories such as "The Open Window" or "The Lull" the victory is achieved by a young girl successfully fooling adults by mental dexterity without causing physical harm.

Nicholas, the main figure in "The Lumber Room", finds himself in a situation much like Conradin's, and in a similar, but less violent fashion, his life consists of minor victories over adults, in which "the 
older, wiser, and better people [are] proved to be profoundly in error in matters about which they had expressed the utmost assurance" (372). The topsy-turvy logic of adults is demonstrated in his aunt's decree that he is not to enter the gooseberry garden because he is in disgrace, eliciting the wonderfully zeugmatic but impeccable logic: "Nicholas did not admit the flawlessness of the reasoning; he felt perfectly capable of being in disgrace and in a gooseberry garden at the same moment" (373). Here again, the reader is asked to sympathise with the child against trivial, irrational and deliberately spiteful domestic restrictions.

Left out of a hastily arranged trip to the sea-side (unlike Conradin, he has relations and a "quite uninteresting younger brother" (372), Nicholas is able to put a plan into action and enter the forbidden lumber-room, "an unknown land, compared with which the gooseberry garden was a stale delight, a mere material pleasure" (374). In what is as much a psychological space as a physical one (Maxey 2005:7), Nicholas is liberated by the sight of unfamiliar delights, all pointlessly stored away to gather dust, "a storehouse of unimagined treasures" in a house otherwise "bare and cheerless" (374). His attention is drawn to an elaborate and neglected tapestry depicting a hunting scene, which becomes "a living, breathing story" as he envisages scenarios to bring the scene to a conclusion (ibid.). Even the books are replete with lavish illustrated plates of birds, more colourful and exciting than the magpies and wood-pigeons with which he is familiar. Suddenly he is returned to reality by the sound of his aunt shouting his name from the gooseberry garden and claiming that she can see him perfectly well. His amused reaction "was probably the first time for twenty years that anyone had smiled in that lumber-room" (375). Having baited her trap, the aunt is now patrolling the garden in the hope of catching Nicholas out, while he savours the marvels that adults keep locked away in a dark and secret place.

On entering the garden, he finds that his aunt has slipped into an empty water-tank and is unable to climb out. This gives him cause to engage in a verbal battle-of-wits by pretending that his interlocutor is the Devil, and to employ her own vindictiveness against her:

"Your voice doesn't sound like aunt's," objected Nicholas; "you may be the Evil One tempting me to be disobedient. Aunt often tells me that the Evil One tempts me and that I always yield. This time I'm not going to yield." 
"Don't talk nonsense," said the prisoner in the tank; "go and fetch the ladder."

"Will there be strawberry jam for tea?" asked Nicholas innocently.

"Certainly there will be," said the aunt, privately resolving that Nicholas should have none of it.

"Now I know that you are the Evil One and not aunt," shouted Nicholas gleefully; "when we asked aunt for strawberry jam yesterday she said there wasn't any. I know there are four jars of it in the store cupboard, because I looked, and of course you know it's there, but she doesn't, because she said there wasn't any. Oh, Devil, you have sold yourself!" (376)

As Nicholas is only too aware, "such childish luxuries are not be overindulged in" and he strolls off, leaving a kitchen-maid to rescue his aunt (376). "Tea that evening was partaken off in a fearsome silence" (ibid.). Ultimate satisfaction is achieved when the trip to the beach turns out to have been a fiasco, and the story ends with Nicholas successfully concocting an escape plan for the hunter on the tapestry in the lumber-room, a sign that his mental freedom of movement has in no way suffered from his 'punishment'.

There is once more a suggestion that Saki's inspiration, or at least the germ of an idea, may have come from his own childhood, as according to Ethel, "the character of the aunt in "The Lumber Room" is Aunt Augusta to the life" and she confirms the accuracy of the painful and silent teas that followed the sudden withdrawal of a treat (Munro,1929: 7). In addition, Langguth's description of the Munros' childhood home includes a forbidden garden with gooseberries and "one enchanted room but, the source of its enchantment, it was always kept locked” (11).

\section{Imagination Appeased}

The concluding story in this analysis differs from the others in that an adult succeeds in providing mental stimulation to children, who consequently do not need to fall back on their own powers of invention. Again, Ethel provides a parallel in Munro's young life. When Hector was twelve, his father employed a governess for his sister, who would also be expected to teach the younger brother. She turned out to be a "real companion" (Munro,1929: 20), who had no trouble with the children 
as she cared for and amused them, much as their father or uncle did on their infrequent visits. Aunt Augusta, however, had her dismissed out of fear that she was "trying to mesmerize her" (Munro,1929:21). She appointed her own successor, but Ethel says nothing of her, so presumably she was cast in the same mould as her employer.

Finding himself tormented by the boredom of two small girls, a boy and an "aunt belonging to the children" (349), all strangers, on a train journey, a nameless bachelor undertakes, at the invitation of the exasperated aunt, to tell a story that the children will "both understand and appreciate" (351). Pursuing her theme of a girl who was saved from a mad bull because people "admired her moral character" (350), he relates the tale of Bertha "who was extra-ordinarily good"(351). Attention begins to waver at this stage until the children hear the unfamiliar oxymoron "horribly good" (ibid.), hinting at an element of excitement absent from all of the aunt's prior endeavours.

Bertha is awarded medals for her obedience, punctuality and good behaviour and given the unique opportunity to visit the Prince's park, which is otherwise barred to children. She wanders the grounds, her medals clinking all the time, until the appearance of a huge wolf: "mudcolour all over, with a black tongue and pale grey eyes that gleamed with unspeakable ferocity. The first thing that it saw in the park was Bertha; her pinafore was so spotlessly white and clean that it could be seen from a great distance" (353).

Compromised by both her moral and sartorial purity, she manages to secrete herself in some myrtle bushes. As she trembles with fear, regretting that her good behaviour has brought her to this predicament, the medals begin to strike each other and the wolf is alerted to her location. "All that was left of her were her shoes, bits of clothing, and the three medals for goodness" (ibid.).

The children respond with praise. The story is the most beautiful ever, or in the young boy's assessment: "It is the ONLY beautiful story I have ever heard" (ibid.), and the bachelor is content that the children have been silenced for ten minutes, despite the aunt's vigorous protestations that he has "undermined the effect of years of careful teaching" (354). Saki is once again firmly on the side of "fanciful concoction against stale reality" (Langguth, 1982: 217), and here exceptionally, the thirst is slated by the fertile imagination of a, presumably, young adult and not 
by children themselves. The man's story succeeds because it recognises the need to admit that violence is an inescapable constituent of life and that virtue does not always prevail, despite the tone of most adults and most of the children's literature they produce.

The final story in this analysis, "The Toys of Peace" re-iterates the message of the "Lumber-Room", i.e. the imagination of the child will endure despite all attempts to repress it, but it also goes some way to explaining the circumstances that led to Munro's death.

In accordance with the newspaper article that prefaces the story, Bertie and Eric are given the latest playthings, "Toys of Peace" These are lead representations of John Stuart Mill, a municipal dust-bin and a model of the Manchester branch of the Young Women's Christian Association, for example, invented to wean boys off soldiers and to curb a growing lust for war and battle. Predictably, the experiment fails when the boys assign everything a more military identity. The YMCA building becomes a besieged fortress and the liberal use of the paintbox and ink results in colourful uniforms or blood-splattered bodies, at which point their well-meaning mother and uncle admit defeat.

So drawn are the boys to the glamour of flags, uniforms, generals and battles from European history that their uncle wonders if it would be possible to rewrite history to concentrate more on socially important turning-points than on battles. The implicit answer is of course in the negative. This aversion to the military is criticised more forcefully in the novel When William Came (1913), most notably at its conclusion when the Boy Scout Movement, "young hearts that had not forgotten, had not compounded, would not yield" (813), becomes the only organised force to offer resistance the German invader.

If there was a price to pay for this attraction, then Munro the adult ultimately paid it. While he had witnessed bloody revolution and conflicts as a journalist, i.e. as a disinterested observer, the outbreak of war in August 1914 provided the opportunity to become involved as a direct participant. True to his belief that all "[n]early every redblooded human boy has had war, in some shape or form, for his first love" (Reynolds, 1919: xviii), he enthusiastically enlisted and choose to serve in the ranks. In his mid-forties at this point, he described the military as "like being boy and man at the same time" (Langguth, 1982: 257). Unlike the harmless games of "The Toys of Peace", however, "the 
romance of a European war" (251) was played out with genuine bullets, and it was one of these that ended the life of Munro, and the career of Saki, in November 1916.

\section{WORKS CITED}

Barrie, J.M.. The Plays of J.M. Barrie in One Volume. London: Hodder and Stoughton, 1928. <https://archive.org/details/plays030673mbp>

Byrne, Sandie . "The Short Stories of Hector Hugh Munro ('Saki')." A Companion to the British and Irish Short Story. Malcolm, Cheryl Alexander and David Malcolm (eds), Oxford: Wiley-Blackwell, 2007.

---. The Unbearable Saki: The Work of H. H. Munro. Oxford: Oxford University Press, 2008.

Dyson, Jeremy . Foreword. Saki: A Shot in the Dark. London: Hesperus Press Limited, 2006.

Langguth, A.J.. Saki, a life of Hector Hugh Munro: With Six Short Stories never before Collected. Oxford: Oxford University Press, 1982.

Malcolm, David. The British and Irish Short Story Handbook. Oxford: WileyBlackwell, 2012.

Maxey, Ruth. "Children are Given us to Discourage Our Better Instincts: The Paradoxical Treatment of Children in Saki's Short Fiction." Journal of the Short Story in English vol. 45 (Autumn 2005), 1-12. Web. 13 July 2013. <www.jsse.revues.org/442>

Milne, A.A.. "Introduction". The Chronicles of Clovis. London: Bodley Head, 1926. <http://www.gutenberg.org/files/3688/3688-h/3688-h.htm>

Munro, Ethel. "Biography of Saki." The Square Egg, and Other Sketches. New York: The Viking Press, 1929. <http://www.unz.org/Pub/MunroHH1929v08>

Nevinson, H.W. Introduction. Beasts and Superbeasts (1914) New York, The Viking Press, 1930.<https://archive.org/details/beastssuperbeast00saki>

Reynolds, Rothay. "A Memoir of H. H. Munro." The Toys of Peace and other Papers. London: John Lane The Bodley Head, 1919. <https://archive.org/ details/toysofpeaceother00sakirich>

Saki (Munro, H. H.). The Penguin Complete Saki. (1976) Harmondsworth: Penguin Books, 1986. 
Salemi, Joseph S. "An Asp Lurking in an Apple-Charlotte: Animal Violence in Saki's The Chronicles of Clovis." Studies in Short Fiction 26 (Fall, 1989): 423-430.

Received: 21 March 2014 Accepted: 03 November 2014 


\section{BOOK REVIEWS}





\title{
VIORICA PATEA, ED. (2012). SHORT STORY THEORIES: A TWENTY-FIRST CENTURY PERSPECTIVE.
}

\author{
Amsterdam: Rodopi, ISBN: 978-90-420-3564-5, 346 pp.
}

\author{
José R. Ibáñez \\ Universidad de Almería
}

Almost fifty years have elapsed since Thomas H. Gullason published an article in which he identified the reasons why the short story was considered an underrated literary form. In his view, the number of practitioners discussing the form and the scarcity of critical essays and books on theory resulted in a lack of interest among American readership (Gullason 1964:17). In the Seventies, Charles E. May edited Short Story Theories, a groundbreaking volume made up of articles by experts on the genre which have come to mark the starting point of recent short story criticism. As might be expected, May disagreed with Gullason and anticipated that the main problem faced by short fiction was partly due to "a reaction to too much systematic criticism in the first few decades of the twentieth century" (May 1976:5). He blamed critics (e.g. Brander Matthews) and writers (e.g. O. Henry) for taking advantage of the popularity of short fiction among readers. Soon, many critics and hack writers rushed to imitate Matthews and O. Henry to secure popular financial success in what resulted in a banalization of the form, a fact that prompted serious readers and critics to call for an end to it.

The following decades witnessed the publication of critical studies which put the genre under scrutiny: Ian Reid's The Short Story (1977), Susan Lohafer's Coming to Terms with the Short Story (1983), Susan Lohafer and Jo Ellyn Clarey's Short Story Theory at a Crossroads (1989), May's sequel The New Short Story Theories (1994), Farhat Iftekharuddin, Mary Rohrberger and Maurice Lee's Speaking of the Short Story (1997) and, finally, Barbara Lounsberry, Susan Lohafer and Mary Rohberger's The Tales We Tell: Perspectives on the Short 
Story (1998) are just some of the many books published. The turn of the century also confirmed a renewed interest in short fiction, although a definite deviation with regard to previous studies was noticed. In this sense, Lohafer, one of the leading-voices in the form, stated that recent "discussions of the short story tend now to be genre-bending and interdisciplinary" (1998:xi). As Laura Lojo (2013:195) rightly points out, the essays published in twenty-first century critical volumes ${ }^{1}$ seemed to attest to Lohafer's prophetic words. The most recent trend in scholarly publications have placed an interest in building academic bridges between Spanish and Anglo-American scholars and researchers in the field. Those are the cases of Contemporary Debates on the Short Story (2006), edited by José R. Ibáñez, José Francisco Fernández and Carmen $\mathrm{M}^{\mathrm{a}}$ Bretones, and more recently, Modernism, Postmodernism, and the Short Story in English (2012), a volume compiled by Jorge Sacido.

The collection of essays edited by Viorica Patea is to date the latest contribution to short fiction criticism and the unmistakable proof that the short story continues to raise a growing interest in the academic community. Patea's remarkable book is a sixteen-essay volume with contributions by Spanish, Spanish American and American scholars who explore the genre from historical, theoretical and practical perspectives. The book title may well be interpreted as an homage paid to May's 1976 and 1994 editions to critical studies on short fiction and his academic efforts in the field. ${ }^{2}$

The compilation is divided into four different sections which aim to cover the most diverse types of approaches to short fiction. It opens with Viorica Patea's introduction that tackles issues concerning the beginnings of the short story, theory of the form, structure and evolution. Patea rightfully points out that the short story is a very ancient literary form, its earliest examples dating back to Biblical times. She acknowledges the importance of Poe - the founding father

${ }^{1}$ Among such outstanding studies, Fahrat Iftekharuddin et al.'s Postmodern Approaches to the Short Story (2003), Charles E. May's The Short Story. The Reality of Artifice, Maurice Lee's Writers on Writing. The Art of the Short Story (2005), Per Winther, Jakob Lothe and Hans H. Skei's The Art of Brevity. Excursions in Short Fiction Theory and Analysis (2007) should be cited.

${ }^{2}$ In this sense, nine out of sixteen contributions published in Patea's anthology have acknowledged, quoted from or articulated their analyses from articles that have appeared in both of May's volumes. 
of the American short story - and the rising interest that the form gained after the period of banalisation at the turn of the twentieth century which rendered disaffection among American readers and critics. In her analysis of the structure of the short story, Patea lingers on the different discussions that have arisen around its nature and its definition as genre, by bearing in mind features such as brevity, fragmentation, intensity and mystery ${ }^{3}$. In addition to this, Patea looks into the evolution of the form beginning with Poe's early conception that made the short story "America's most identifiable homegrown literary genre" (16). Thus, if Poe laid the foundations of the genre, the short story evolved in the hands of Anton Chekhov whose stories were characterized by an impressionistic atmosphere and a "minimal dependence on the traditional notion of plot" (May 1994:201), and was soon echoed by modernist writers (Joyce or Mansfield).

Antonio López Santos's study aims to prove that medieval tales may help us understand the development of the modern short story as he analyses Chaucer's Canterbury Tales as unique narrative pieces not comparable to other medieval practitioners such as Giovanni Boccaccio (The Decameron, 1353) or John Gower (Confessio Amantis, 1390) (28). In his study, López Santos offers an insightful reading of Chaucer's "The Wife of Bath's Prologue and Tale" and "The Nun's Priest's Tale" from a narratological perspective and concludes by reasoning that these sample tales possess certain features, namely the tree-like structure, that allow multiple interpretations which "seem closer to the modern short story than to the classic tale" (45).

Peter Gillian's "Anticipating Aestheticism: The Dynamics of Reading and Reception in Poe" focuses on the American author's peculiar vision of Aestheticism in "The Oval Portrait" and "The Fall of the House of Usher". Gillian offers a remarkable attempt to explain how Poe's aesthetic vision, characterized as "a form of haunting - a return of something that had been repressed" (50), was welcomed in Europe and eventually transformed with completely new features before its transatlantic return. In this sense, Poe was welcomed in Europe with open arms and became the key figure shaping European Aestheticism -

${ }^{3}$ Eudora Welty stated that "the first thing we notice about our story is that we can't really see the solid outlines of it - it seems bathed in something of its own. It is wrapped in an atmosphere... [which] may be its chief glory" (1976:163). 
in Britain influencing Dante Rossetti, Robert Louis Stevenson, or Oscar Wilde. In France, however, French Aestheticism had developed out of Baudelaire's response to Poe; French "Décadence", on the other hand, emerged "as the exploration of abnormal... the psychopathology of modern life" (69). Thus, when Poe's aesthetism returned to America in the 1890s, the uncanny effect which it produced in American artists was even stranger to the "haunting" effect produced in Poe's contemporaries.

In "Revising Theory: Poe's Legacy in Short Story Criticism", Erik Van Achter broaches a fundamental problem which has long remained unsolved since Poe's day. The short story has always been and "continues to be defined in relation to other genres," primarily the lyric and the novel, and "continues to resist or elude definition" (77). Van Achter contends that short story criticism after Poe is sterile. Thus, what became a stack of notes which were not intended to become a poetics of the new genre had held sway for over more than a century. Van Achter determines the existence of two main waves of short story theories during the twentieth century: the first one, which hardly moved away from Poe's framework, sprung up after Brander Matthews' The Philosophy of the Short Story and spread out until the end of the era dominated by the New Criticism (77); a second wave of short story criticism, developed from the Seventies onward, strove to overcome Poe's authoritative presence as critics regarded his short story framework "as an obstacle to be surmounted" (78).

The second section of the book brings to the foreground the short story under the conspicuous scrutiny of discourse analysis, cognitive theories and pragmatism. This part opens with the study entitled "Frames Speaking: Malamud, Silko, and the Reader" by the late Per Winther, to whom the volume is dedicated. Winter examines Bernard Malamud's "The Magic Barrel" in relation to two stories by Leslie Marmon Silko by applying the notion of framing that "has proved itself analytically fruitful in the hands of a number of short story critics" (89). Winther diverges from Teun A. van Dijk's articulation of global structures in discourse as well as Ian Reid's set of framing categories so as to offer alternative readings which go beyond the fairy-tale traditional analysis of Malamud's anthologized story. In "A Cognitive Approach to Short Story", Pilar Alonso compares long and short narratives produced by the same authors and examines samples by John Updike and Eudora Welty. In consonance with cognitive theoreticians, 
Alonso claims that "the unique quality" that both authors claim "for the imagined worlds and their cognitive processes" resides in the skillful way "they obtain exceptional artistic results by performing" the same type of cognitive operations and linguistic devices employed by most of us in communication (114-5). Alonso concludes by pinpointing the benefits obtained in the application of cognitive operations as key factors in the development of narrative genres.

In "Code-Switching as a Strategy of Brevity in Sandra Cisneros' Woman Hollering Creek and Other Stories" Consuelo Montes-Granado examines the use of Spanish words and phrases in Cisneros' stories and recalls how the Spanish language had become a symbol of resistance in Chicano literature in the 1960s and 1970s. Montes-Granado states that the need to reach a wider readership prompted some Chicano writers to turn to English as the vehicle of expression in their writing. The presence of code-switching in Cisneros may thus be regarded as tokens or symbols, the use of "Spanish words or short phrases" becoming a sort of "brushstrokes of paint in the hands of an artist - unobtrusive, but loaded with meaning" (136).

In "The Yellow Hybrid: Gender and Genre in Gilman's Wallpaper" Carolina Núñez Puente analyzes Charlotte Perkins Gilman's "The Yellow Wallpaper" from a hybrid approach based on the femininity and sexuality of the protagonist and the gothic and realist nature of the narrative. Bearing in mind the hybrid nature of Gilman's text termed as diary, short story or autobiography - Núñez Puente falls back on Bakhtin's notion of dialogics, articulated by the Russian author in novels written by male authors, and applies it to this short story written by a woman. In the case of Gilman's short story, Núñez Puente demonstrates that the hybrid voices perceived in the narration belong to the narrator and the "author's voice" that contradicts her words (143).

Rebeca Hernández explores the limits of storytelling in "Short Narrations in a Letter Frame: Cases of Genre Hybridity in Postcolonial Literature in Portuguese" as she examines three narratives in Portuguese which may have traditionally not been regarded as short fiction. In this sense, Hernández analyzes Mozambican writer Luís Bernardo Honwana's letter "Rosita até morrer", Angolan writer António Jacinto's "Carta de um contratado" and a letter included in the novel Chiquinho by Cape Verdean writer Baltasar Lopes so as to prove that 
"irrespective of the genre these letters adopt" all those examples share "functional conditions" which affect the reader "in the same way the short story does" (156). The importance of this study lies, however, in the hybrid nature of these texts based on the orality which enables authors with the possibility of fusing orality with marginality in order to create a subversive style which shakes off their social subjugation to the metropolis.

The study penned by María Jesús Hernáez Lerena sets up a connection between short fiction and testimony literature. In both forms of fiction, knowledge is challenged making it almost impossible to get an intelligible explanation of our experience (175). Hernáez Lerena departs from the notion of visual shock and the subsequent paralysis of rational thinking undertaken by Walter Benjamin and Susan BuckMorss and their correspondence to testimony literature, characterized by an eyewitness. The short story, as Hernáez Lerena contends, manifests a strong dependence on the visual, a fact that drives readers to be defined as onlookers or witnesses, rather than interpreters or 'knowers'.

In her contribution to the volume, Teresa Gibert surveys Margaret Atwood's short fiction through the use of the metaphorical conceptualization that this Canadian author deploys in her fiction. Gibert examines certain devices, such as metaphorical strategies or the figurative language as a way to "enhance the reader's awareness of literariness and to stimulate closer inspection of her short stories as artifacts" (206). She concludes her study by acknowledging that Atwood's experimental short fiction applies subversive methods, namely metafiction, intertextuality, magical realism, and points out that those metaphorical strategies may also respond to an interest of language playfulness which also increases the artificial quality of her literary texts.

In "Body Politics: Female Dynamics in Isabel Allende's The Stories of Eva Luna" Farhat Iftekharuddin claims a risky assumption: the short story reflects the feminine better than the novel does while at the same time he focuses on what he believes is a central aspect of the former: the concept of enigma. By addressing that the short story as a genre "captures the sublime within the female body and psyche" (225), Iftekharuddin engages in an analysis in which he defends the 
subversion of patriarchal hegemony perceived in Isabel Allende's The Stories of Eva Luna. For him, the characters created by Allende are merely constructs, self-created and entrepreneurs, whose powers are to be found in the politics of language and body which target the male characters for an active and cognitive rediscovery of their female counterparts.

The fourth and last section is devoted to postmodern approaches to the short story. In this regard, Luisa María González Rodríguez's contribution explores Donald Barthelme's short fiction "as complex intertextual spaces where collage and intertextuality work on the metafictional paradox between the construction of a fictional reality and the laying bare of that illusion" (251). According to González Rodríguez, Barthelme articulates such devices in order to challenge social, political and aesthetic modes and structures. In "Realism and Narrators in Tobias Wolff's Short Stories", Santiago Rodríguez Guerrero-Strachan analyzes the role of narrators, the different possibilities of the narrative voices and the minimalist techniques used by Wolff in three short stories. In his insightful reading, Rodríguez Guerrero-Strachan exposes the fundamental role of narrators and their reliability as well as the role of the reader, who becomes a key element in the interpretation of the fragmented story, which Ewing Campbell denominated as "truncated plot" (1998:15), one of the key features of minimalist stories. In "The Boundaries of Serial Narrative", Lauro Zavala ventures to vindicate the role of the Spanish language as lingua franca of mini-fiction during the second half of the twentieth century. He supports the claims of those critics who have affirmed that French was the lingua franca of the novel during the second half of the nineteenth century while English became the lingua franca of the short story during the first half of the twentieth century. In this vein, Zavala articulates a taxonomical approach to South American long and short fiction narratives, many of which, due to their particular nature, namely fantastic bestiaries or dispersed short stories, may not have clear counterparts in the literature produced in English.

The volume ends with a panoramic overview of the early twentyfirst-century American short fiction by Charles E. May, probably the best-known and most acclaimed critic of this genre. In his article "The American Short Story in the Twenty-first Century", May surveys the American short-fiction at the end of the millenium and the turn of the 
century in an attempt to assess the new directions of the genre after the 1970s and 1980s 'renaissance'. He claims that after the denigration of minimalism, the short story regained its former ancillary position as a fictional form and "once more [it] languished in the shadow of the novel" (300). In his overview, May pays a fitting visit to the fiction of Bobbie Ann Mason, Ann Beattie or Tobias Wolff; he then turns to acknowledge the active role of brand-new voices such as ZZ Packer, Kevin Brockmeier, Ryan Harty or Julie Orringer, whose narratives strive to set up new directions in American storytelling.

In accordance with current critical studies on the short story, Patea's compilation pays proper tribute to the genre. In it, some articles successfully delve into the history and main features of the form, whereas other contributions offer critical reflections of the short story at the beginning of the new millenium. Despite the fact that the pioneering studies by Charles E. May and Susan Lohafer still loom large in many of the articles in this volume, it seems fitting to acknowledge that Patea has successfully achieved a suitable, enjoyable and thoughtprovoking combination of critical contributions produced by some of the most acclaimed scholars and younger voices in the field, a collection that may well soon become a remarkable landmark in the development of short story criticism.

\section{WORKS CITED}

Campbell, Ewing. "How Minimal is Minimalism?" The Tales We Tell. Perspectives on the Short Story. Ed. Barbara Lounsberry et al. Westport, CT: Greenwood Press, 1998.

Ibáñez, José R., José Francisco Fernández, Carmen $\mathrm{M}^{\mathrm{a}}$ Bretones. Contemporary Debates on the Short Story. Bern: Peter Lang, 2007.

Iftekharuddin, Farhat, Mary Rohrberger and Maurice Lee. Speaking of the Short Story. Interviews with Contemporary Writers. Jackson: University Press of Mississippi, 1997.

Gullason, Thomas H. "The Short Story: An Underrated Art?" Studies in Short Fiction. 2:1 (Fall 1964): 13-31. 
Lohafer, Susan. "Introduction." The Tales We Tell. Perspectives on the Short Story. Westport, CT: Greenwood Press, 1998. ix-xii.

Lohafer, Susan \& Jo Ellyn Clarey, ed. Short Story Theory at a Crossroads. Baton Rouge: Louisiana State University Press, 1989.

Lojo Rodríguez, Laura María. Review of Short Story Theories: A Twenty-first Century Perspective, by Viorica Patea (2012). Atlantis. Journal of the Spanish Association of Anglo-American Studies, 35.2 (December 2013): 195-201.

Lounsberry, Barbara, et al., ed. The Tales We Tell. Perspectives on the Short Story. Westport, CT: Greenwood Press, 1998.

May, Charles E., ed. Short Story Theories. Athens: Ohio University Press, 1976.

---. ed. The New Short Story Theories. Athens: Ohio University Press, 1994.

---. The Short Story. The Reality of Artifice. New York \& London: Routledge, 2002.

Reid, Ian. The Short Story. The Critical Idiom. London: Methuen \& Co Ltd., 1977.

Sacido Romero, Jorge, ed. Modernism, Postmodernism, and the Short Story in English. Amsterdam: Rodopi, 2012.

Welty, Eudora. "The Reading and Writing of Short Stories". Short Story Theories. Ed. Charles E. May. Athens: Ohio University Press, 1976. 159-177.

Winther, Per, Jakob Lothe, and Hans H. Skei, ed. The Art of Brevity. Excursions in Short Fiction Theory and Analysis. Columbia: The University of South Carolina Press, 2004.

Received: 21 July 2014 Accepted: 05 November 2014 



\title{
TIMOTHY M. COSTELLOE, ED. 2012. THE SUBLIME: FROM ANTIQUITY TO THE PRESENT.
}

\author{
Cambridge: Cambridge University Press, \\ ISBN: 978-0-521-19437-2 (hardback), ISBN 978-0-521- \\ 14367-7 (paperback). xiv + 304 pp EDUARDO (2009).
}

\author{
Jonathan P. A. Sell \\ Universidad de Alcalá
}

The sublime as philosophical idea, psychopathological experience, aesthetic quality and objective phenomenon receives extensive coverage in the essays Timothy M. Costelloe, whose excellent The British Aesthetic Tradition: From Shaftesbury to Wittgenstein was published recently, has edited under the title The Sublime: From Antiquity to the Present. Handsomely produced with abundant black and white illustrations, the book is a veritable thing of beauty considering the current cash-strapped times. But its beguiling surface cannot conceal a disfigurement that no historical survey of the sublime can avoid, namely the gaping 1,400year hole in conventional histories between Longinus (3rd century $\mathrm{CE}$ ) at one end and 350 years of commentary and analysis initiated by Nicolas Boileau's Traité du Sublime (1674) at the other. Yet as Costelloe notes in his own chapter on "Imagination and Internal Sense", ideas cognate to Longinus' were available in Plato's Symposium and Plotinus' Ennead I and familiar in the seventeenth century to the Cambridge Platonists (50-1). More importantly, if, as Costelloe states in rebuttal of recent obituarists (Elkins), the sublime is "a human experience" as well as "a philosophical concept" (1), then it "can no more disappear than the experience to which it refers" (7). Indeed that experience is attested to in the evidence from antiquity and early modernity marshalled in Richard A. Etlin's chapter on "Architecture and the Sublime" and Éva Madeleine Martin's on "The 'Prehistory of the Sublime in Early Modern France: An Interdisciplinary Perspective". But for a chronological survey to be complete, there is still a great deal of gap filling to be done. 
The insistence of Costelloe's introduction on the phenomenological sublime dovetails neatly with Malcolm Heath's chapter, "Longinus and the Ancient Sublime", which opens the first part of the volume ("Philosophical History of the Sublime") and emphasises the "heretical" (5, quoting Monk 29-31) nature of Boileau's style-centred reading of his source. Heath sensibly rehabilitates Cassius Longinus as author of $\mathrm{On}$ Sublimity (15-16) before showing how Longinus' treatise sows the seeds of the Romantic sublime as it ranges from sublimity of discourse, through sublimity in nature, to the sublimity of metaphysical transcendence. We are therefore primed for the leap forward in time to Edmund Burke, the subject of Rodolphe Gasché's chapter "... And the Beautiful? Revisiting Edmund Burke's 'Double Aesthetics". Gasché's deployment of the sublime as foil to his painstaking discussion of the beautiful and the terror/love binary alerts us to the fact that this book is not intended for beginners. On the other hand, his claim that "from today's perspective, beauty is perhaps the intrinsically more important and stimulating part of Burke's aethetics" (26) is never explained or substantiated, leaving the chapter heavy in analysis but light in conclusions. For its part, Melissa McBay Merritt's chapter, "The Moral Source of the Kantian Sublime", assumes the reader's acquaintance with Kant's thinking on the sublime. Merritt argues closely that Kantian pleasure in sublimity, which is a state of mind, derives from our awareness that deficient "sensible representation" can be compensated by the power of reason and thus enacts the dichotomy between our animal and rational selves. Similarly, "sound moral disposition exercises a certain sublimity of mind" (39) in which an animal rejection of morality is countered by a rational attraction towards it.

After this initial ascent of the three major peaks of the philosophical sublime, Costelloe's own chapter 4, "Imagination and Internal Sense: The Sublime in Shaftesbury, Reid, Addison and Reynolds" considers competing eighteenth-century empiricist (internal sense) and, so to speak, "idealist" (imagination) conceptions of the sublime and, by extension, aesthetics. Of particular interest is Costelloe's championing of Joshua Reynolds as an acute theorist of the sublime whose emphasis on the visual nature of the imagination anticipates Wordsworth and the Romantics (63). In similar spirit, Rachel Zucker delineates a third "associative" conception of the sublime in Alexander Gerard, Lord Kames, Archibald Alison Alison and Dugald Stewart. Contra Burke, 
Zucker's quartet agreed that the pain or terror generated by natural phenomena could not be equated with the sublime if those phenomena were not also expressive of something (for example, of the Creator's power). Thus, art objects could be "equally or more sublime than natural objects" (69) and, in line with Kant's relocation of the sublime to the perceiving subject, "our experience of the sublime is fundamentally active, brought about by our power of imagination" (69). Éva Madeleine Martin's chapter, already cited, offers among much fascinating treasure trove an analysis of the iconology of the experience of the sublime in seventeenth-century French painting (88-101). At the same time she shows how recent French scholarship, with Marc Fumaroli at its head, has traced a Longinian tradition stretching back from Boileau through Guez de Balzac, Nicolas Poussin, Pierre Corneille, Torquato Tasso, Joseph Scaliger, Michel de Montaigne, and Pierre Ronsard, to Francesco Robortello's first printed edition of Peri hupsous of 1554. What is more, she demonstrates how the category of the sublime could be subjected to various, sometimes conflicting, cultural appropriations exploiting the sublimis / humilis binary implicit in it. For example, the genre painting bestowed transcendence on the meanness of life outside refined circles (85-88) in an innovation analeptic of Wordsworth and Coleridge's Lyrical Ballads or, possibly, Julia Kristeva's abjection.

Meanwhile, the quasi-religious anéantissement or self-nullifying amazement which Martín identifies as one strand of the early modern French sublime looks forward to Schopenhauer's view that contemplation of the Ideas "releases [the individual] from the circle of desire and frustration characteristic of ordinary selfhood" and into an existence as "pure subject", as Paul Guyer puts it (113) in the following chapter on "The German Sublime After Kant". From Kant's cognitivist take on the sublime and Schiller's specialisation of it "into a property of art rather than of nature" (107), Guyer leads us tidily through Schelling's and Hegel's reversion to an objectivist sublime of "magnitude or infinitude of realities" (102), to Schopenhauer's conversion of the sublime into the experience of "natural beauty achieved in the face of nature's hostility to individual existence" (102) and finally Nietszche's identification of the it, once more confined to art, as the experience first of the transcendence of rationality rather than of any Kantian "transcendent rationality" (102), and then, after Schopenhauer, of the "transcendence of individuality" (117). Equally difficult texts come under scrutiny in 
David B. Johnson's dense chapter on "The Postmodern Sublime", which tries to find a coherent pattern in the disparate writings on the subject of Jean-François Lyotard, Gilles Deleuze, Julia Kristeva and Frederic Jameson, all of whom are shown to agree that the experience of the sublime is sparked by the crisis of representation entailed by the inaccessibility of particular objects to the imagination, or "faculty of presentation" (118-119), but to differ in what they take those objects to be: an idea of the absolute (Lyotard), the passage from chaos to "the trans-sensible rhythm of sensation" (Deleuze, 127), the experience of abjection (Kristeva) and "the postmodern inability to marshal a critique of ... 'late capitalism"' (Jameson, 129). While Lyotard and Kristeva offer some food for thought, Deleuze mistakes explanatory metaphors (most strikingly, the "body without organs") and Jameson grandiose generalisations for matters of fact.

The second part of this slightly arbitrarily divided collection ("Disciplinary and Other Perspectives") opens with John R. J. Eyck's chapter on "The 'Subtler' Sublime in Modern Dutch Aesthetics", which covers the period 1730-1835. Contra Christophe Madelein's thesis that Dutch thinking on the sublime was largely reactive and post-Kantian, Eyck argues for an earlier and more original contribution. His analysis of Balthazar Huydecoper's Essay on Language and Poetry (1730), Rhijnvis Feith's The Ideal in Art (1821) and Jacob Geel's Talk atop the Drachenfels (1835) leads to the rather unsubstantiated conclusion that the "subtler" Dutch sublime is peculiarly culture-specific, prizing the practical and modest over the shocking and the awesome-what Martin might have defined as protestant humilitas. More convincing in its depiction of a historically-sited discourse of the sublime is Chandos Michael Brown's chapter on "The First American Sublime". Ranging widely from Jonathan Edwards and Thomas Jefferson to Henry Thoreau and James Fenimore Cooper, Brown delineates the specificities of a discourse required to ply a careful line between Puritan religiosity and Enlightenment rationalism when representing the vast and sometimes forbidding grandeur of the New World "wilderness". In the process, a distinctly non-metropolitan identity was consolidated and the revolutionary cause advanced.

That American obsession with landscape leads to Emily Brady's chapter on "The Environmental Sublime", which is vitiated by a penchant for "technography" (a favourite word of Heath's), muddy 
thinking and green ethics which would endow nature with rights of its own. At times the difference between artistic and natural sublimes is one of degree, scale or intensity (177), at times of quality, the artistic sublime being a second-order or metaphorical replica of the natural sublime (173). Brady's denunciation of the anthropocentrism of discourse on the natural sublime is hard to square with her touting of cognitivist Kant as philosophical mentor of the environmental sublime. Otherwise, the sublime is diluted first into a "sense of 'otherness", then into "mystery", both to no apparent purpose. That man must always figure in representations of the sublime is clear from the extracts from the Bible and the Bhagavad-Gita which serve as epigraphs to Andrew Chignell and Matthew C. Halteman's chapter on "Religion and the Sublime". Technographically impeccable, like Brady's the chapter betrays a terminological anxiety which errupts into the following taxonomy: "the theistic sublime", the "spiritualistic sublime" —each of which are subcategorisable as "corroborative", "conversional" or "transformational"-, the "demythologistic sublime" and the "nontheistic sublime", precipitated by the experience either of "evil as privation" or "as abjection". To their credit, Chignell and Halteman conclude by registering a measure of scepticism regarding the very existence of any such thing as the sublime, echoing James Elkins to the effect that "no one has a firm grasp on the context" (201). For his part, in his chapter on "The British Romantic Sublime" Adam Potkay takes the same "ethical turn" as Heath, Merritt and Brady in theirs. In a nutshell: "In the British Romantics ... the sublime of transcendence is related and finally subservient to the moral sublime" (205. Original Emphasis). Acknowledging Longinus' seminality in this respect, and admitting of Unitarian, quasi-Christian and "heroic" moralities (207), Potkay analyses fragments of Byron and Worsdworth before restating his thesis in considerably weaker terms ("Much needs to be said about re-envisioning the Romantic sublime as, at least in part, the moral sublime", 215. My emphasis) and proposing further areas of enquiry.

Theodore Gracyk's chapter "The Sublime and the Fine Arts" ranges widely in its attempt to theorise the non-representational sublime embodied in such abstract art as Mark Rothko's Seagram Murals. As well as providing one answer to the problem of representability intoned by Leotard, Deleuze, Kristeva and Jameson-all of whom on Gracyk's account would needlessly require sublime art to be mimetic of reality- 
neglected sources such as Charles Batteaux's The Fine Arts Reduced to a Single Principle (1746), James Beattie's Essays on Poetry and Music and They Affect the Mind (1776), William Crotch's Substance of Several Courses of Lectures on Music (1831) and E.T.A. Hoffman's essay "Beethoven's Instrumental Music" are given a deserved dusting down. The collection culminates in Richard A. Etlin's splendidly sprawling chapter on "Architecture and the Sublime" which celebrates the "somoesthetic" effect, a term borrowed from Richard Shusterman (12 ), wrought by architecture's peculiar fusion of the "spatial sublime" (implicit in Longinus) with the "cosmological sublime". Etlin's discussion of literary sources and architectural works spans classical Rome, Byzantium and Renaissance Italy and France, not to mention Homer, Dante, Milton and Étienne-Louis Boullée's late eighteenth-century projects for the Metropolitan Church and a Cenotaph to Newton. Together with Martin's chapter, Etlin's is a reminder that more may be learned from hands-on analysis than abstruse theorisation.

Less, then, a historical survey of the sublime than a high-definition snapshot of the current state of relevant academic art, The Sublime: From Antiquity to the Present is a mine of ideas and sources. While reaffirming Kant's centrality to current theoretical discussion, it also reinstates Longinus as much more than a mere stylist. Indeed, the sublime is revealed to be so vital in all senses of the word that both its modish refashioning as an ethical principle and the anxiety over its identity turn out to be as misplaced as well-intentioned. Though inevitably uneven, Costelloe's collection will become indispensable for any serious student of the subject and should set the agenda for work on the sublime for years to come.

\section{WORKS CITED}

Costelloe, Timothy M. The British Aesthetic Tradition: From Shaftesbury to Wittgenstein. Cambridge: Cambridge University Press, 2013.

Elkins, James. "Against the Sublime." Roald Beyond the Finite: The Sublime in Art and Science. Eds. Roald Hoffman and Iain Boyd White. Oxford: Oxford University Press, 2011. 
Fumaroli, Marc. L'age de l'éloquence. Geneva: Droz, 1980.

Madelein, Christophe. "Juigchen in den adel der menschlijke natuur: Het verhevene in de Nederlanden (1770-1830)." PhD dissertation, Vakgroep Nederlandse literatur, University of Ghent, 2008.

Monk, Samuel Holt. The Sublime: A Study of Critical Theories in XVIII-Century England. Ann Arbor: University of Michigan Press, 1935.

Schusterman, Richard. Body Consciousness: A Philosophy of Mindfulness and Somaesthetics. Cambridge: Cambridge University Press, 2008.

Received: 23 June 2014 Accepted: 09 September 2014 



\section{NOTES ON THE AUTHORS}



Alexandra Bikkyová is a $\mathrm{PhD}$ candidate at the Department of British and American Studies at Pavol Jozef Šafárik University in Košice, Slovakia. She is conducting research for her doctoral thesis on Gender in 20th Century American Literature and specializes in the concept of Perversion in Chuck Palahniuk's chosen novels. She also teaches courses on gender studies and British and American media, and is the author of several articles within these research fields.

Macarena García-Avello has a $\mathrm{PhD}$ in 20th Century American Literature at University of Oviedo and an Erasmus Mundus Master degree in Women's and Gender Studies (GEMMA) at University of Bologna and University of Oviedo. Currently, she works at University of Maryland. Her research is centred in 20th Century British, Canadian and American Literature, Feminist Theory and the Representation of Gender in Fiction. She is the author of the books "While Men Sleep..." Malestar e identidad de género en Revolutionary Road (2011) and Producción / contraproducción de las identidades de género en Los puentes de Madison (2011).

Ángeles García Calderón is a Tenured Lecturer at the Department of Translation and Interpretation, Romance Languages, Semitic Studies and Documentation of the University of Córdoba (Spain).

M. Carmen Gómez Galisteo holds a B.A. in English with honors and a Ph.D. (sobresaliente cum laude) in American Studies, both from the Universidad de Alcalá. She is the author of The Wind is Never Gone: Sequels, Parodies and Rewritings of Gone With the Wind (McFarland, 2011) and Early Visions and Representations of America: Alvar Núñez Cabeza de Vaca's Naufragios and William Bradford's Of Plymouth Plantation (Bloomsbury, 2013). She currently teaches at UNED (Universidad Nacional de Educación a Distancia) in Madrid (Spain).

Aitor Ibarrola Armendáriz teaches courses in migrant fiction, ethnic relations and film adaptation at the University of Deusto, Bilbao, Spain. He has published articles and edited books on minority and immigrant narratives, as well as processes of cultural hybridisation. Currently, he is the Director of the MA Program in Migrations and Social Cohesion (MISOCO) and Chair of the Modern Languages and Basque Studies Dept. in the Faculty of Social Sciences and Humanities at the UD 
María J. López is Senior Lecturer at the University of Córdoba. She has published Acts of Visitation: The Narrative of J.M. Coetzee (Rodopi, 2011) and in journals such as the Journal of Southern African Studies, the Journal of Commonwealth Literature, English Studies in Canada, Atlantis, English in Africa and Journal of Literary Studies.

Beatriz Martínez Ojeda is a Ph. Doctor and Honorary collaborator at the Department of Translation and Interpretation, Romance Languages, Semitic Studies and Documentation of the University of Córdoba (Spain).

Sean Moran teaches English in the Faculty of Applied Linguistics and Translation Studies at the University of Gdansk in Poland. He is currently researching a doctoral thesis on the suffragist, author and actress Cicely Hamilton. This is his first published article.

Jonathan P. A. Sell lectures in English at the University of Alcalá, Spain. He is author of Rhetoric and Wonder in English Renaissance Travel Writing, 1560-1613 (2006), Allusion, Identity and Community in Recent British Writing (2010) and Conocer a Shakespeare (2012), and editor of Metaphor and Diaspora in Recent Writing (2012). He is currently researching early modern ideas of the sublime and preparing a Spanish edition of Florence Farmborough's Life and People in National Spain.

Susana Vega González lectures in the English Department at the University of Oviedo, Spain. She holds a Ph.D. and has published extensively on African American literature, with a special focus on Toni Morrison's fiction. Among her publications are the book Mundos Mágicos: la otra realidad en la narrativa de autoras afroamericanas (Servicio de Publicaciones de la Universidad de Oviedo, 2000) and the article "Toward a Love Ethic: Love and Spirituality in bell hooks's Writing," included in Critical Perspectives on bell hooks (Routledge, 2009). 


\section{NOTES FOR CONTRIBUTORS}





\section{NOTES FOR CONTRIBUTORS}

The Grove. Working Papers on English Studies is a peer reviewed, MLA indexed periodical. Published annually and distributed both nationally and internationally, The Grove is sponsored by the research group HUM. 271 of the Regional Andalusian Government and is published by the University of Jaén (Spain).

The major scope of The Grove is literatures in English, critical theory, English language and linguistics, translation, English as a foreign language and cultural studies.

The Editor kindly invites submissions in English or Spanish of original unpublished articles and book reviews within the domain of the above topics, as well as unpublished poems or short literary contributions.

Editorial correspondence and submission of articles and book reviews for publication should be addressed to:

The Editor

The Grove. Working Papers on English Studies

Departamento de Filología Inglesa, Facultad de Humanidades y CC.EE. Edificio D-2

Universidad de Jaén, Campus Las Lagunillas s/n

23071 Jaén, Spain.

You may also write to grove@ujaen.es; Editors' phone numbers: 953212609 / 953212136 / 953211827.

\section{Contributions should be unpublished and not considered for publication elsewhere.}

\section{Reviewing process}

The submitted papers will be considered for publication if they receive favourable reports from specialists in the author' research field. At least two anonymous referees (at least one of them from a university other than that of Jaén) will evaluate the unsigned manuscripts submitted for publication to The Grove.

Decisions on articles and book reviews submitted are normally made within one month and those accepted will be published within one year. If the paper has not been accepted or needs revision, the author will be provided with a report indicating the reasons for non-acceptance, or the changes required to be made for its publication. 


\section{Selection of contributions}

Given that The Grove receives more submissions (articles, reviews and fiction/poetry) than it can publish, our peer-reviewers are asked to consider the quality and originality of each paper accepted for publication. In general terms, to be acceptable a paper must: deal with one of the fields of study covered by our journal, be original, provide substantial evidence for its conclusions, and prove relevant for the specific field. Additionally, we will be looking for methodological rigour, theoretical consistency and innovation, stylistic merit and academic seriousness. Typically, an unsolicited paper will be sent to two peer-reviewers and may be approved, rejected, or approved with modifications, in which case detailed reports will be sent to the author(s) for its potential consideration; the paper will be evaluated again unless the editors consider the modification of minor importance. Modifications suggested to the author(s) may include (among others) requests to revise the style of the paper, reorganize the material, provide additional evidence or reinforce/clarify either the initial hypothesis or the conclusions. Author(s) will be requested to include all title, abstract and keywords in Spanish and English. The final publication of the papers, and the section where they will appear, will be determined by the editorial board.

\section{PUBLICATION GUIDELINES FOR CONTRIBUTORS}

The suggested length of articles is 3000 to 6000 words and book reviews from 1500 to 2500. All articles should be accompanied by a 100-150 word abstract and by the title of the work both in English and in Spanish. Below the abstract should appear the key words which the author/s would like to include also in the two languages. Manuscripts should be sent in Word format to grove@ ujaen.es in a separate attachment to a covering letter. All details of the author - title of the text, name of author, institution, academic / professional post, telephone numbers, postal and email address, as well as a brief résumé in English of 50 words maximum - should be included in a separate attachment as a cover sheet, never on the manuscript itself. The author should never write in first person in the text or notes if these references help to identify the author. Submissions should be prepared according to the MLA Style Manual and Guide to Scholarly Publishing (latest edition) throughout.

- Contributors must not write their names on the article, but on a separate cover sheet, together with their affiliation(s), mailing and email addresses and telephone number(s) as well as the title of the article. Only if the article has been accepted for publication must they write their names and institution in the final version of the article, between the title and the abstract.

- All submissions must be the author's own, original work.

- Articles and book reviews may be written in either Spanish or English.

- Articles should not normally exceed 10-20 pages (3000-6000 words) 
- Articles must be accompanied by a 5 to 10 line long abstract in English followed by $5-10$ key words. The abstract and key words should be translated into Spanish.

- Book reviews should be between 1500 and 2500 words in length.

- The style should conform to the MLA Style Manual and Guide to Scholarly Publishing 2005.

- Manuscripts must be submitted in digital form in Word format as a file attachment to their submission through email.

- The contributor should specify that the article submitted is not being considered for publication elsewhere.

After a positive evaluation, manuscripts not conforming to the guidelines provided will be returned to the authors for further revision.

\section{Citations}

Double quotation marks should be used for text quotations, while single quotes should emphasise a word or phrase or highlight its figurative meaning. Only foreign words and titles of monographs may appear in italics. If exceeding four lines, block quotes should be separated from the main text and the whole quotation indented 2,54 cm (1") on its left margin.

References should include the page numbers or, if the author is not mentioned earlier in the paragraph, the surname(s) of the author(s) plus the page numbers. Examples:

References embedded within the main text (four lines maximum):

In his work, "Fiedler focused on Shakespeare only, and he included women and 'Indians' ...", while in my analysis I will include a wider corpus of early modern English texts (10) or (López-Peláez 10).*

*If more than one work by the same author is included in the bibliography, the citation should include the first word(s) of the title of the book/article: (Strangers 10) or (López-Peláez, Strangers 10).

Block quotes (five or more lines):

... the Spanish monarchs Isabel and Fernando were simultaneously campaigning to defeat the last Iberian stronghold of Islam, the kingdom of Granada. The year they succeeded, 1492, was also the year in which they obliged Spain's remaining Jews to convert to Christianity or emigrate. Ten years later Muslims were given the same choice. After another century of tensions Philip III moved to expel all Moriscos in 1609. (Burns 188-89)

If part of the original text is omitted, three dots without brackets should be included. 


\section{Bibliographical References. Examples:}

Author's surname(s), Author's name(s), and 2nd Author's name(s) 2nd Author's surname(s). Title. Original publication date. Edition. Volumes. Place: Publisher, Year.

\section{Monographs:}

Duiker, William J., and Jackson J. Spielvogel. The Essential World History, Volume 2. 2005. 6th. ed. 2 vols. Boston: Wadsworth, 2011.

\section{Multiple works:}

Follett, Ken. Lie Down with Lions. New York: Signet, 1986.

---. The Pillars of the Earth. New York: Signet, 1990.

Citations: (Lie Down 123) or (Follett, Lie Down 123); (Pillars 123) or (Follett, Pillars 123)

\section{Edited book / Chapter:}

Kavanagh, James H. "Shakespeare in Ideology." Alternative Shakespeares. 1985. 2nd ed. Ed. John Drakakis. London: Routledge, 2002. 147-69.

López-Peláez, Jesús. Foreword. Strangers in Early Modern English Texts. Ed. Jesús López-Peláez. Frankfurt: Peter Lang, 2011.

\section{Translated book:}

Eco, Umberto. The Name of the Rose. Trans. William Weaver. New York: Harcourt, 1983.

\section{Two or more authors:}

Greer, Margaret R., Walter D. Mignolo, and Maureen Quilligan. Rereading the Black Legend. Discourses of Religious and Racial Difference in the Renaissance Empires. Chicago: University of Chicago Press, 2007.

Rivara, Frederick P., et al. "Prevention of Bicycle-Related Injuries: Helmets, Education, and Legislation." Annual Review of Public Health 19 (1998): 293-318.

\section{Article:}

Solé, Yolanda. "Valores aspectuales en español." Hispanic Linguistics 4.1 (1990): $57-85$.

\section{Reviews:}

Camhi, Leslie. "Art of the City." Rev. of New York Modern: The Arts and the City, by William B. Scott and Peter M. Rutkoff. Village Voice 15 June 1999: 154.

\section{Online Journal:}

Barry, John M. "The Site of Origin of the 1918 Influenza Pandemic and Its Public Health Implications." Commentary. Journal of Translational Medicine 2.3 (20 Jan. 2004): 1-4. Web. 18 Nov. 2005. <http://www.translationalmedicine.com/content/2/1/3>.

\section{Websites:}

Research Project: Muslims, Spaniards and Jews in Early Modern English Texts: The Construction of the 'Other'. Ed. Jesús López-Peláez. University of Jaén. Web. 21 Oct. 2011. <http://www.ujaen.es/investiga/strangers/index.php>. 


\section{Further guidelines:}

- The font Times New Roman should be used in the whole manuscript.

- The first line (only) of each paragraph should be indented 1,27 cm (0,5").

- Footnotes should be kept to a minimum, and bibliographic references should be avoided.

- Double inverted commas should be used for "Titles of articles" or "Quotes embedded within running text”; simple inverted comas for 'Emphasis'; and italics for Book Titles and Foreign Words.

- Bold font should be used for headings and subheadings only.

- Abbreviations such as pp., i.e., e.g., etc., should be avoided. Use instead: that is; for example; and so on.

- When page numbers are used for citation, they should be included within parenthesis and without abbreviations such as p. or pp. The format 100 08 is preferred instead of $100-108$ or $100-8$.

- Style should be coherent throughout the whole text: British or American English.

- Long dashes should be used for additional comments, and the spaces between dash and comment should be removed. 





\section{the grove 21 - 2014 \\ INDEX}

Foreword

THE CONCEPT OF PERVERSION IN CHUCK PALAHNIUK'S NOVEL CHOKE

Alexandra Bikkyova .

"THE SYNAPSES COLLAPSE; WELL, LET THEM..." EL FEMINISMO POSTMODERNO DE THE STONE DIARIES DE CAROL SHIELDS

Macarena Garcia-Avello

UN CASO NOTABLE DE TRADUCCIÓN INDIRECTA: THE LAST OF THE MOHICANS DE FENIMORE COOPER TRADUCIDO POR VICENTE PAGASARTUNDUA A TRAVÉS DEL FRANCÉS

Ángeles García Calderón, Beatriz Martínez Ojeda

HOUSES WITH PICKET FENCES: TRYING TO RECOVER A PAST THAT NEVER WAS IN PLEASANTVILLE AND CELEBRATION, FLORIDA

M. Carmen Gómez Galisteo

CANLITERARY WORKS HELPTO MEMORIALIZE NATURAL DISASTERS? ZORA NEAL HURSTON'S THEIR EYES WERE WATCHING GOD

Aitor Ibarrola-Armendáriz.

"THEIR TRAVELS WERE REAL TRAVELS": HISTORY AND FICTION IN J.M. COETZEE'S "THE NARRATIVE OF JACOBUS COETZE" AND IN EUROPEAN EXPLORATION NARRATIVES IN SOUTHERN AFRICA

María J. López.

VENGEANCE DEFERRED: CHILDREN IN THE SELECTED SHORT STORIES OF SAKI

Sean Moran

\section{BOOK REVIEWS}

VIORICA PATEA, ED. (2012). SHORT STORY THEORIES: A TWENTYFIRST CENTURY PERSPECTIVE

José R. Ibáñez.

TIMOTHY M. COSTELLOE, ED. (2012). THE SUBLIME: FROM ANTIQUITY TO THE PRESENT

Jonathan P. A. Sell

BIOGRAPHIC NOTES ON THE AUTHORS

NOTES FOR CONTRIBUTORS. 